\title{
IMPROVED WHEEL PERFORMANCE ON SAND BY CONTROLLED CIRCUMFERENTIAL RIGIDITY
}

by

\section{K. W. Wiendieck}

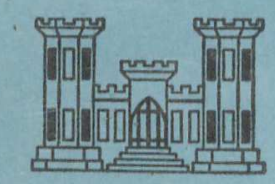

May 1969

\section{Sponsored by \\ Assistant Secretary of the Army (RED) \\ Department of the Army}

\section{Conducted by}

U. S. Army Engineer Waterways Experiment Station CORPS OF ENGINEERS

Vicksburg, Mississippi

THIS DOCUMENT HAS BEEN APPROVED FOR PUBLIC RELEASE AND SALE; ITS DISTRIBUTION IS UNLIMITED 
Destroy this report when no longer needed. Do not return it to the originator.

The findings in this report are not to be construed as an official

Department of the Army position unless so designated by other authorized documents. 


\section{IMPROVED WHEEL PERFORMANCE ON SAND BY CONTROLLED CIRCUMFERENTIAL RIGIDITY \\ by}

K. W. Wiendieck

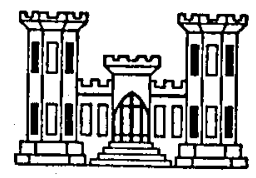

May 1969

Sponsored by

Assistant Secretary of the Army (R\&D)

Department of the Army

Conducted by

U. S. Army Engineer Waterways Experiment Station CORPS OF ENGINEERS

Vicksburg, Mississippi

ARMY-MRC VICKSBURG. MISS.

THIS DOCUMENT HAS BEEN APPROVED FOR PUBLIC RELEASE AND SALE; ITS DISTRIBUTION IS UNLIMITED 
$n=4$

(1) M-69-2

cap. 2

THE CONTENTS OF THIS REPORT ARE NOT TO BE USED FOR ADVERTISING, PUBLICATION, OR FROMOTIONAL PURPOSES. CITATION OF TRADE NAMES DOES NOT CONSTITUTE AN OFFICIAI ENDORSFMEINT OR APPROVAL OF TIE USE OF SUCH COMMERCIAL PRODUCTS. 
. 
FOREWORD

The study reported herein was funded by Department of the Army Project 4A013001A91D, "In-House Laboratory Independent Research (ILIR) Program," Item T, sponsored by the Assistant Secretary of the Army (R\&D). The study was conducted during the period 1966-1968.

The project was conceived by Dr. Klaus W. Wiendieck of the Mobility Research Branch (MRB), Mobility and Environmental (M\&E) Division, at the U. S. Army Engineer Waterways Experiment Station. (WES). The experimental wheel used in the project was constructed under contract by WIVE, Inc., Chestertown, Md. The test program was carried out by personnel of the MRB under the general. supervision of $\mathrm{Mr}$. W. J. Turrbull, former Technical Assistant for Soils and Envirronmental Engineering, and Messrs. W. G. Shockley and S. J. Knight, Chief and Assistant Chief, respectively, M\&: Division; and under the direct supervision of Dr. D. R. Freitag, Chief, MRB. The report was prepared by Dr. Wiendieck.

COI John R. Oswalt, Jr., CE, and COL Levi A. Brown, CE, were Directors of the WES during this study. Mr. J. B. Tiffany and Mr. F. R. Brown were Technical Directors. 
4 
CONTENTS

$\underline{\text { Page }}$

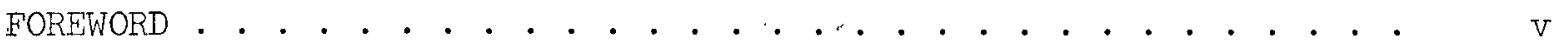

NOTATION . . . . . . . . . . . . . . . . . . . . . . . . . . . . ix

CONVERSION FACTORS, METRIC TO BRITISH AND BRITISH TO METRIC UNITS

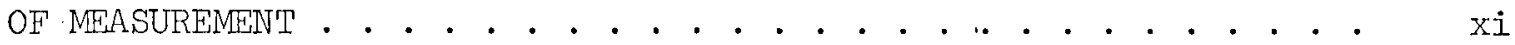

SUMMARY. . . . . . . . . . . . . . . . . . . . . . . xiii

PART I: INTRODUCTION. . . . . . . . . . . . . . . . . . . . . I I

Background . . . . . . . . . . . . . . . . . . 1

Purpose. . . . . . . .. . . . . . . . . . . 4

Scope. . . . . . . . . . . . . . . . . . . . . . . 4

PART II: THEORETICAL CONSIDERATIONS . . . . . . . . . . . . . . . 5

The Normal Stress Distribution Pattern as a Function of Local

Rigidity Variation . . . . . . . . . . . . . . . . . .

Numerical Evaluation . . . . . . . . . . . . . . . 12

PART III: DESCRIPTION OF THE EXPERIMENTAL WHEEL AND TEST

PROCEDURES. . . . . . . . . . . . . . . . . 20

Experimental Wheel . . . . . . . . . . . . . . . 20

Test Procedures. . . . . . . . . . . . . . . . . . . 30

PART IV: ANALYSIS AND DISCUSSION OF TEST RESULTS. • • • . • • • • • 33

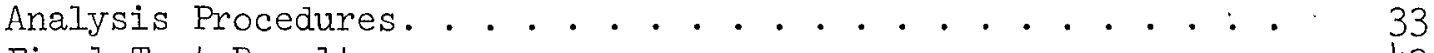

Final Test Results . . . . . . . . . . . . . . . . . 43

PART V: CONCEPT FOR A MORE EFFECTIVE WHEEL. . . . . . . . . . . . . 57

PART VI: CONCLUSIONS AND RECOMMENDATIONS. . . . . . . . . . . . . 62

Conclusions. . . . . . . . . . . . . . . . . 62

Recommendations. . . . . . . . . . . . . . . 62

IITERATURE CITED . . . . . . . . . . . . . . . . . . . . . • 64

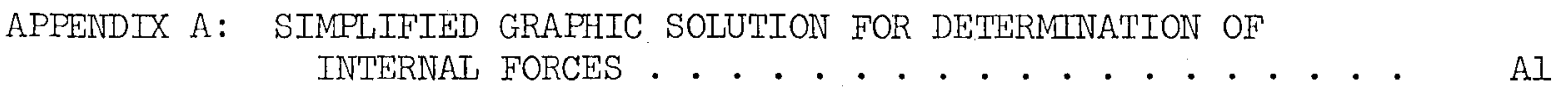



. 
NOTATION

B Wheel width, $\mathrm{cm}$

CI Cone index, psi

Ii Efficiency, dimensionless

$F_{i}$ Friction force at joint $i, \mathbb{N}$

G Penetration resistance gradient, $\mathrm{N} / \mathrm{cm}^{3}$

$k_{s}$ Coefficient of friction between rubber and polished steel, dimensionless

$\ell$ Horizontal projection of forward part of contact length, cm

$\mathrm{M}$ Torque, $\mathrm{m}-\mathrm{N}$

$N$ Resultant of normal stress, $N$

$N_{h}$ Horizontal component of resultant of normal stress, N

$N_{i}$ Normal component of resultant of piston force, $N$

$N_{v}$ Vertical component of resultant of normal stress, $N$

$\mathrm{p}$ Applied air pressure, $\mathrm{N} / \mathrm{cm}^{2}$

$\mathrm{p}_{i}$ Inflation pressure, $\mathrm{N} / \mathrm{cm}^{2}$

P Pull, N

$P_{i}$ Piston force at joint i, $N$

$R$ Wheel radius, $\mathrm{cm}$

$R_{i}$ Resultant force at joint i, $N$

s Slip, percent

$S_{i}$ Force in sliding shoe at joint $i, N$

$t$ Shear to normal stress ratio, dimensionless

$T$ Resultant of tangential stress, $N$

$\mathrm{T}_{\mathrm{h}}$ Horizontal component of resultant of tangential stress, $\mathbb{N}$

$\mathrm{T}_{\mathrm{i}}$ Tangential component of resultant of piston force, $\mathbb{N}$

$\mathrm{T}_{\mathrm{V}}$ Vertical component of resultant of tangential stress, $\mathbb{N}$ 


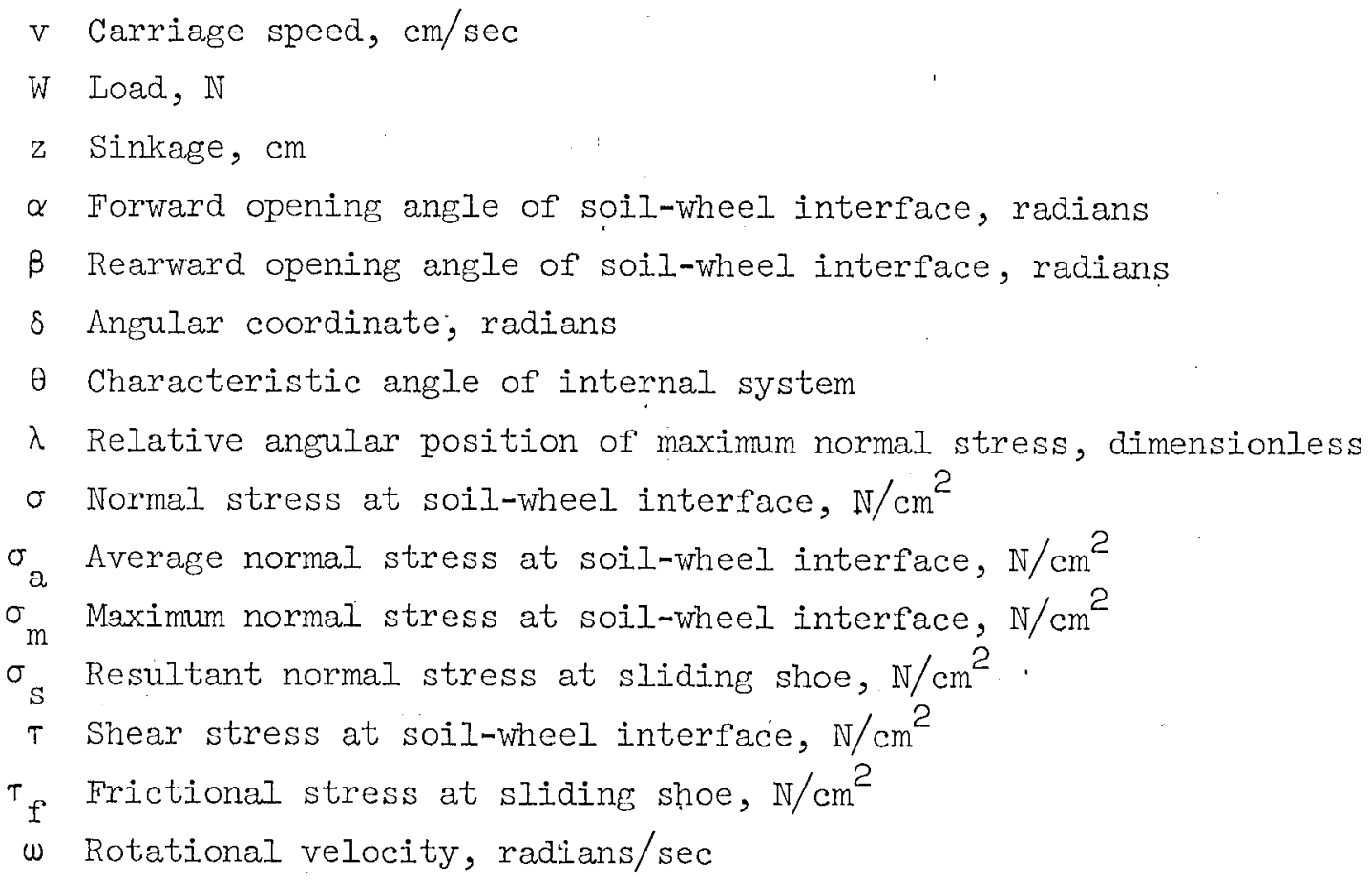


CONVERSION FACTORS, METRIC TO BRITISH AND BRITISH TO METRIC UNITS OF MEASUREMENT

Metric units of measurement used in this report can be converted to British units as follows:

\begin{tabular}{llll}
\multicolumn{1}{c}{ Multiply } & By & & \multicolumn{1}{c}{ To Obtain } \\
centimeters & 0.3937 & & inches \\
meters & 3.2808 & feet \\
Newtons & 0.225 & pounds \\
meter-Newtons & 0.7376 & foot-pounds \\
Newtons per square centimeter & 1.4503 & pounds per square inch \\
penetration resistance gradient & 11.0253 & \\
$\quad$ in Newtons per cubic centimeter & &
\end{tabular}

British units of measurement used in this report can be converted to metric units as follows:

\begin{tabular}{|c|c|c|}
\hline Multiply & By & To Obtain \\
\hline inches & 2.54 & centimeters \\
\hline feet & 0.3048 & meters \\
\hline pounds & 4.4482 & Newtons \\
\hline foot-pound.s & 1.3558 & meter-Newtons \\
\hline pounds per square inch & 0.6895 & Newtons per square centimeter \\
\hline $0-6$ in. cone index & 0.0907 & $\begin{array}{l}\text { penetration resistance gradient } \\
\text { in Newtons per cubic centimeter }\end{array}$ \\
\hline
\end{tabular}





\section{SUMMARY}

An analysis of experimentally determined normal stress distributions beneath tires on sand revealed a strong tendency for the normal stresses to be greater in areas of higher-than-average tire rigidity. This phenomenon was most pronounced when the soil was strong and the inflation pressure was low.

Since wheel performance is determined ultimately by the stresses at the soil-tire interface, the question arose as to whether the observed phenomenon could be used advantageously in off-road mobility research. If the stress distribution at the interface depended largely on the rigidity distribution, then a control of the interface rigidity pattern should permit a favorable influence on the stress distribution, and thus an increase in the performance of the wheel.

To examine this hypothesis, an experimental wheel was built; its performance is described in this report. The control of local tire rigidity was achieved by a system of six nonrotating hydraulic jacks inside the wheel acting against a fixed flexible shoe, along which the inner surface of the tire slid as it rotated. By loading the jacks individually in a given pattern, an interface rigidity distribution similar to this loading pattern was achieved.

Stress concentrations at the rear end of the contact patch are obviously favorable, while those at the front end are unfavorable. When the loading pattern of the hydraulic jacks was arranged accordingly in a roughly linear manner, tests with sand at two strength levels showed that the wheel with favorable rigidity distribution (high internal pressure at the rear of the soil-wheel interface) developed up to 25 percent more pull than the wheel with unfavorable rigidity distribution. The greater pull was paralleled by an efficiency increase of the same order. An analysis of the interface geometry, which varies with the internal pressure distribution, showed that the performance variation was accounted for by the interface rigidity pattern alone; no influence of the interface geometry itself could be detected. Another important result of the tests was that only a slight rigidity variation was found necessary for maximum performance improvement. A rigidity variation beyond a given limit was ineffective. However, the rather crude test device did not allow a quantitative assessment of this threshold.

The experimental results were checked by theoretical computations 
based on the variation of assumed soil reaction pressure distribution as a function of the interface rigidity pattern. Both the order of magnitude of the measured performance variations and the observed trends are explained theoretically in this report.

The experimental wheel was built to verify a hypothesis and not to be used as a practical means of locomotion. In fact, it is highly impractical and inefficient in itself, and the performance of conventional tires could not be matched. However, since only very slight rigidity variations are needed to obtain the highest performance, the prospect of applying the principle of controlled rigidity in practice seems promising. Accordingly, a schematic drawing of a multicompartment tire operating upon this principle is presented herein. 


\section{IMPROVED WHEEL PERFORMANCE ON SAND BY}

\section{CONTROLLED CIRCUMFERENTIAL RIGIDITY}

\section{PART I: INTRODUCTION}

\section{Background}

1. If enough power is available and the dynamic vehicle response is not a limiting factor, the performance of a wheeled vehicle depends entirely on the geometry of the soil-wheel interface and the soil reaction stresses at this interface. The complex mechanism, that determines on one hand the geometry of the soil-wheel interface (sinkage, tire deformations), on the other hand the entire stress situation (orientation, magnitude, and distribution of stresses), and thus ultimately the performance of the wheel, is best described by the term "soil-wheel interaction." The engine and transmission of a vehicle serve only to activate this interaction, so wheel. performance improvements seem possible only by interfering with this mechanism in a favorable manner.

2. Although the complex phenomena of the soil-wheel interaction are far from being fully understood, the mechanism is contingent upon the soil properties, the axle load, the torque, and the tire properties. Soil properties and load are generally given quantities that cannot be changed. Torque input and tire properties thus remain for a study of possible wheel performance improvements. Since torque can be varied only in quantity, but not in quality (at least for "soft-soil mobility" problems), the possibil-" ities for vehicle performance improvement over the torque input are believed to be limited.

3. Interfering with the soil-wheel interaction by changing the tire properties is less simply assessed. Variations of carcass structure, tire dimensions, and inflation pressure can be combined into a virtually infinite number of combinations. Theoretical prediction of how such variations of tire properties influence the wheel performance are impossible at the present state-of-the-art, since the physical insight into the mechanism of soil-wheel interaction is rather rudimentary. In fact, only for rigid 
wheels, the geometry of which is particularly simple, have some attempts been made to assess the physical phenomena in the neighborhood of the soilwheel interface and to develop theoretical concepts. ${ }^{1}$ Therefore, the softsoil mobility research with tires has been essentially empirical.

4. The relations between inflation pressure and pull/1oad ratio might serve to illustrate this point. One of the established facts of mobility research is that the pull/load ratio increases with decreasing inflation pressure for sands, if all other parameters are constant. Lowering the inflation pressure not only changes the geometrical conditions of the problem (increased size and a more horizontal overall orientation of the contact patch, and less sinkage), but also alters drastically the entire stress picture at the soil-wheel interface. The normal pressure distribution has been measured and shown to change from a one-peak type for high inflation pressures (the maximum stress being near the center of the contact surface) to the reverse situation for low inflation pressures (a depression in the central part surrounded by a ridge of high pressures along the periphery of the contact surface). 2,3

5.. Little is yet known about the tangential stresses, which are less easily determined; but the tangential stress distribution likely experiences equally drastic changes when the inflation pressure is decreased. The decrease of inflation pressure thus constitutes a severe interference with the soil-wheel interaction, the results of which are unforeseeable at present by theoretical means. As a matter of fact, since the stresses at the soil-wheel interface make a vehicle move, an explanation of increasing performance with decreasing inflation pressure by reference to the interface geometry only is insufficient and even misleading, because it is only a partial explanation that omits reference to the alteration of the stress system. The fact that lowering inflation pressure does increase the pulI/ load ratio means only that the resulting stress system is not unfavorable, or at least, that the possibly unfavorable variation in the stress system is not important enough to offset the effect of the obviously improved geometrical conditions.

6. The experimental wheel with controlled circumferential rigidity, described herein, is a radically new means of interfering with soil-wheel 
interaction. Modification of tire properties (dimension, carcass structure, inflation pressure, tread pattern, etc.) in previous studies might be interpreted as imposing an overall condition on soil-wheel interaction. Local conditions at the soil-wheel interface are not controlled, so that the magnitude and orientation of local interface stresses are left entirely to the mechanism of the interaction within the framework of the overall conditions.

7. The next logical step is to impose specific local conditions and to control, in a more purposeful manner, tire properties or their variations along the soil-wheel interface. The question then arises as to which particular tire property should be controlled locally for performance improvements. Theoretical reasoning and experimental evidence, presented later in this report, led to the conclusion that the tire rigidity (stiffness) pattern at the soil-wheel interface has a major effect on the normal stress distribution. Consequently, the concept evolved that a control of local tire rigidity would allow direct alteration of the normal stress distribution so as to change it in a favorable manner, and thus improve the wheel performance.

8. This report describes the performance of a special experimental wheel built to evaluate this concept, which proved to be successful in that the wheel performance could be influenced to a certain degree. The explanation for this, presented herein, is necessarily insufficient because it is based on the radial stress distribution alone and neglects other and possibly more important facets of the soil-wheel interaction. The explanation provides, however, a kind of qualitative understanding of the phenomena observed.

9. The point is emphasized that the wheel described herein is not intended to be used for soft-soil mobility in the field. It was built to test a hypothesj.s rather than to serve as a practical means of locomotion. However, if the favorable effects of controlled rigidity are deemed worthy of pursuing in a practical sense, a line from this experimental wheel to the development of a practicable soft-soil mobility tire can be drawn. 


\section{Purpose}

10. The purpose of the study was threefold:

a. To investigate, experimentally the extent to which the performance of pneumatic tires on sand can be.influenced by a controlled variation of the tire rigidity within the soilwheel interface.

b. To provide qualitative theoretical reasons to explain the observed performance variations.

c. To present and discuss the concept of a multicompartment tire as a practicable means of applying the idea of controlled circumferential rigidity for cross-country mobility improvement.

\section{Scope}

11. Both theoretical and experimental studies were conducted. The theoretical investigations were general in nature, assuming typical radial stress distributions. The experimental testing focused on programmed increasing-slip tests on an air-dry mortar sand at two strength levels $\left(G=2.72\right.$ and $\left.4.53 \mathrm{~N} / \mathrm{cm}^{3}\right) .^{*}$ The interpretation of the test results was concentrated on the maximum pull/load ratio, and on the efficiency of the wheel as a load transportation device at maximum pull conditions. Various rigidity levels and rigidity variations were investigated.

12. Although the theoretical analysis was based on radial stress. distribution patterns at the soil-wheel interface, no attempt was made to actually measure these stresses. This would have added too much complexity to the already complex test device.

* A table of factors for converting metric to British and British to metric units of measurement is presented on page $x i$. 
The Normal Stress Distribution Pattern as a

Function of Local Rigidity Variation

13. A possible relation between local tire rigidity and normal stress at the soil-wheel interface was mentioned by Freitag and Green: 4

A ridge of high pressure which probably results from the transmission of the load through the relatively stiff tire sidewalls is found to exist at each side of the contact patch. There is a tendency, also, for an area of relatively high stress to exist at the leading and trailing edges of the contact area.

Although this statement specifically refers to tires on unyielding surfaces, stress measurements on yielding soils under tires with low inflation pressures show the same phenomenon of stress concentration at the periphery of the soil-wheel interface, particularly on sand. 2,3,5

14. Results of tests with a given tire at the same inflation pressure are summarized for different sand strength levels in figs. 1 and 2 .
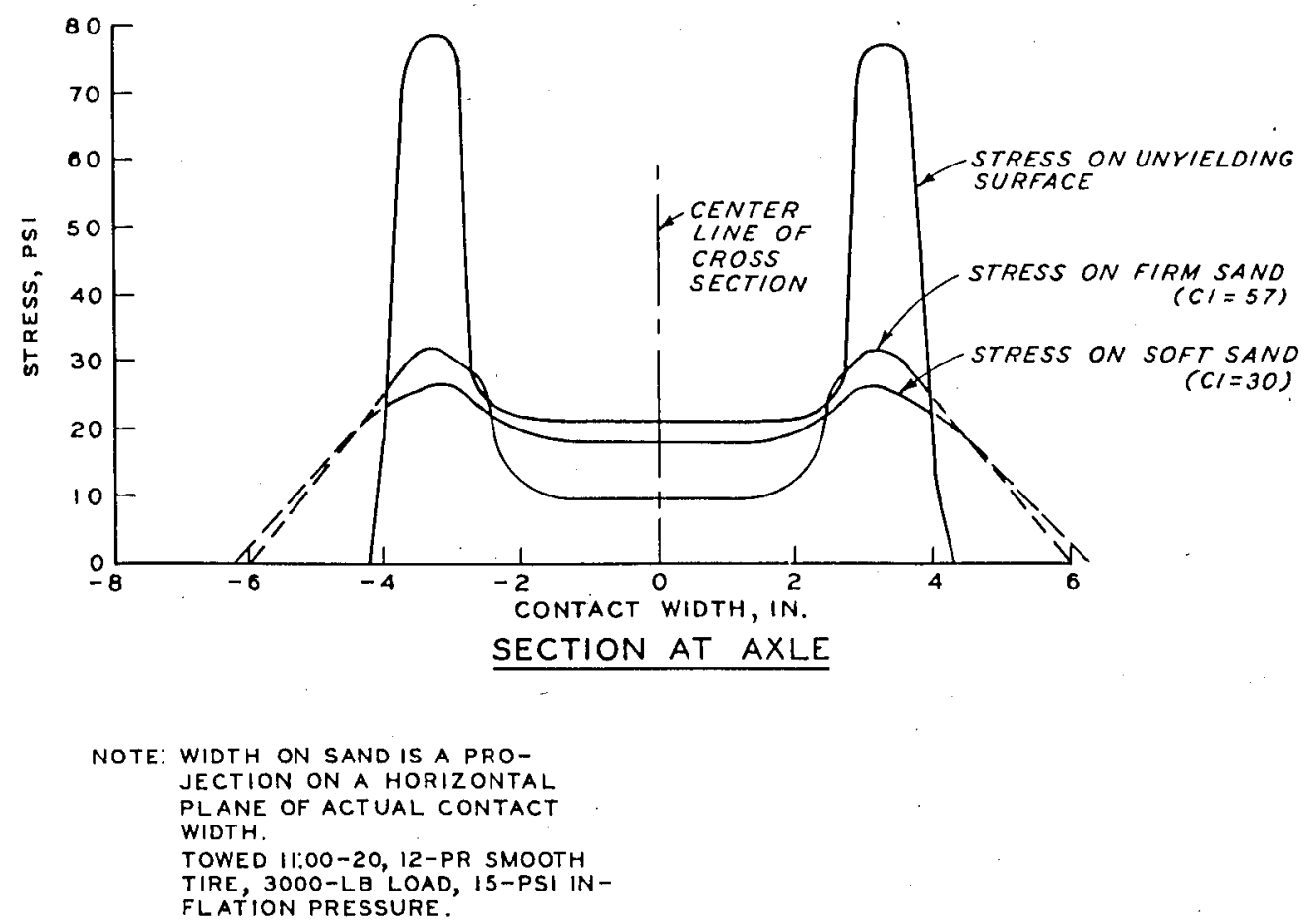

Fig. 1. Distribution of normal stresses across tire width 


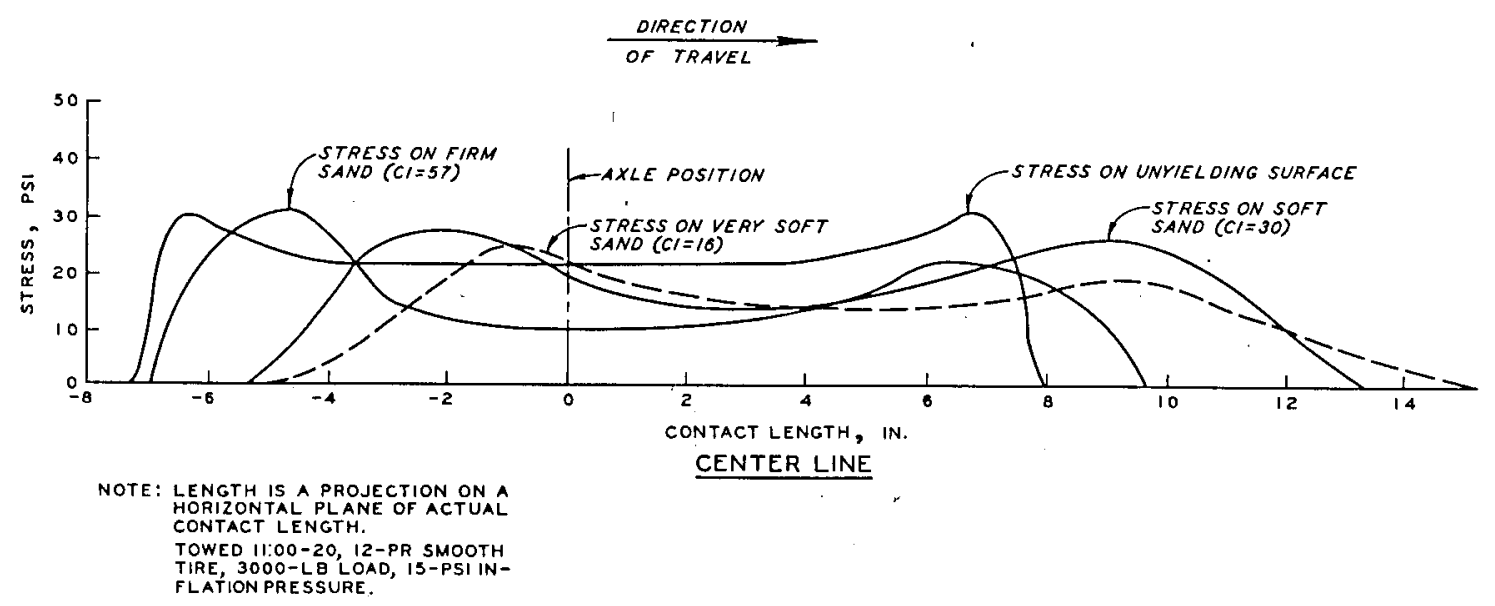

Fig. 2. Distribution of normal stresses along the center line of contact surface (figure from reference 2; dashed line from reference 3)

The pressure concentration along the border of the soil-wheel interface is clearly recognized, this phenomenon being less pronounced in the softer soils. In qualitative terms, the results can be characterized as follows :

a. The softer the soil, the lower the peripheral maximum stress.*

b. The softer the soil, the lower the maximum variation of stress (inclination of curves).

c. The softer the soil, the lower the $\sigma_{\max } / \sigma_{\min }$ ratio.*

15. No attempts have been made to explain these systematic variations in references 2, 3, and 5; and, in particular, no reference has been made to tire stiffness in connection with the peripheral stress concentration. However, the peak stresses occurring at the leading and the trailing edges of the interface have been interpreted in reference 5 as being associated with the flexing of the tire. This, at least, is an indirect reference to rigidity, since a flexed portion of the tire (leading and trailing edges) is stiffer with respect to radial deformations than a plane portion.

16. Summarizing these considerations, three components appear to contribute to the local tire rigidity: (a) inflation pressure,

* With the minor exception of the stress curve on unyielding surface in fig. 2, which is an extreme condition. 
(b) sidewall stiffness, and (c) tire flexing. In general, the rigidity of the central part of the soil-tire interface is governed by inflation pressure alone. The leading and trailing edges exhibit an increased rigidity due to additional tire flexing, the highest rigidity occurring along the lateral edges to which all three factors contribute concurrently.

17. Thus, the stress concentrations in figs. 1 and 2 occur in areas that possess a higher-than-average rigidity. For the tire on an unyielding surface, this is true in an absolute sense in that the stresses near the lateral edges are the highest, and in the center the lowest, with a medium stress occurring at the leading and trailing edges of the interface. For the tire on sand, such differentiation cannot be made; there is no significant difference, for a given soil strength, between the magnitude of the stress at the lateral edge and that of the stresses at the front and rear ends. The rear-end stresses seem even slightly higher than those near the lateral edges.

18. To explain this, the influence of tire rigidity must be examined with respect to the soil strength, and certain limits of the local maximum stresses that the soil is able to sustain must be assumed within the frame of the soil-wheel interaction. Beyond these limits the rigidity variation is ineffective. These limiting maximum stresses obviously decrease with soil strength, which explains the tendency of the stress curves to level out with decreasing soil strength.

19. The same argument can be used with regard to the maximum stress variations (inclination of the stress curves). Normal stress variations are closely related to shear stresses. The lower the shear strength, the less stress variation the soil can sustain; this also contributes to the smoother appearance of the stress curves for the low-strength soils. In the extreme case of a material without any strength (from the soil mechanics viewpoint), such as water, tire rigidity variations would have no influence on the stresses, these being solely determined by hydrostatic conditions.

20. It would be misleading, however, to conclude from this interpretation that the stress distribution tends to follow, within certain limitations, the relative rigidity distribution--although this is exactly the 
case for the data presented in figs. 1 and 2. For example, the rigidity of an undeformable (rigid) wheel is evenly distributed, yet the radial stresses are far from being uniform (fig. 3). Also, edge effects similar

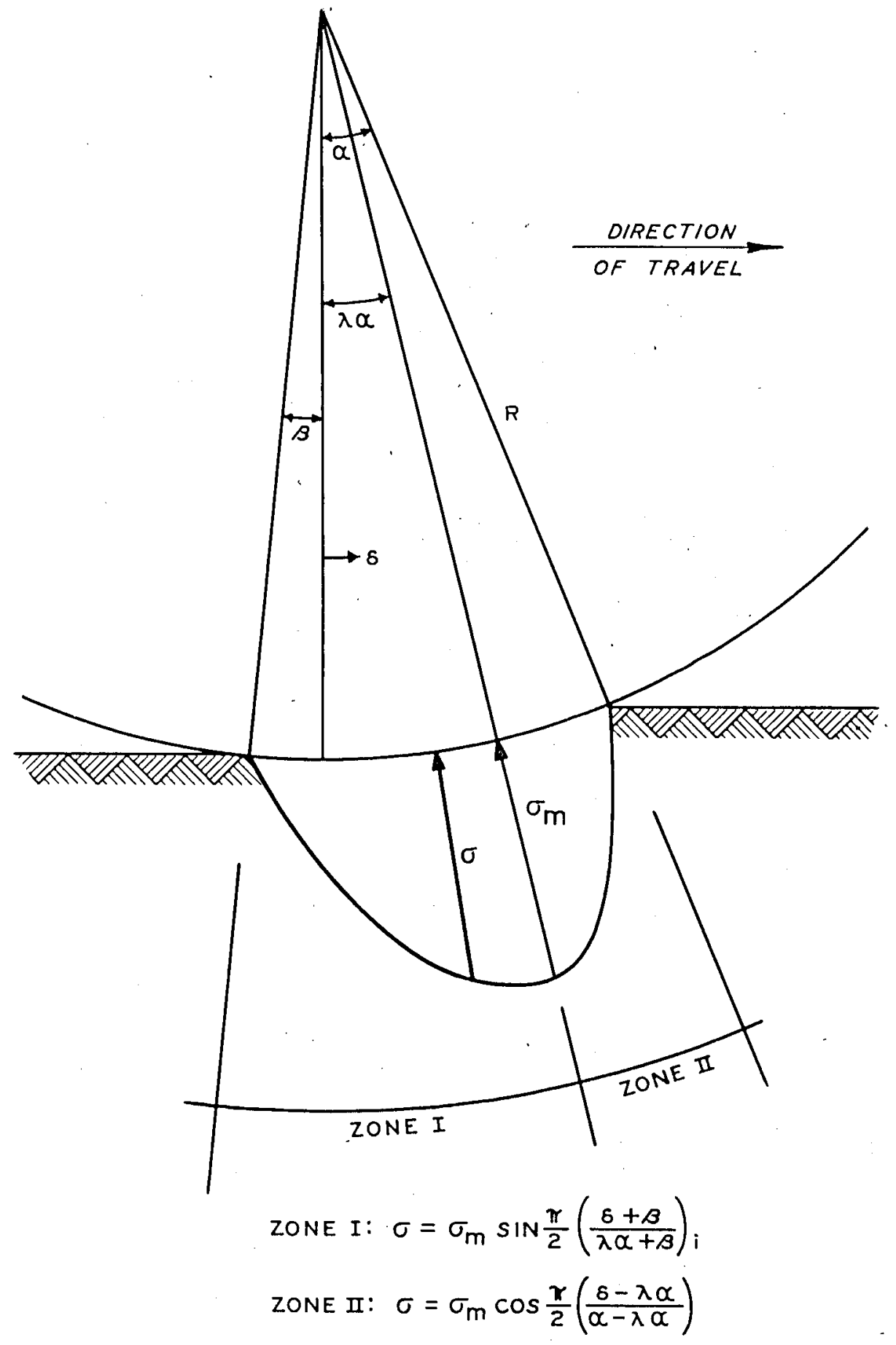

Fig. 3. Representative interface stress for rigid wheel 
to those observed beneath tires have been found to exist beneath circular steel plates being pushed into loose sand (fig. '4). This cannot be attributed to rigidity variations over the plate surface, and is probably due to arching phenomena in the sand.

Fig. 4. Stress distribution beneath rigid plate in sand (unpublished

U. S. Army Engineer Waterways Experinient Station test results)

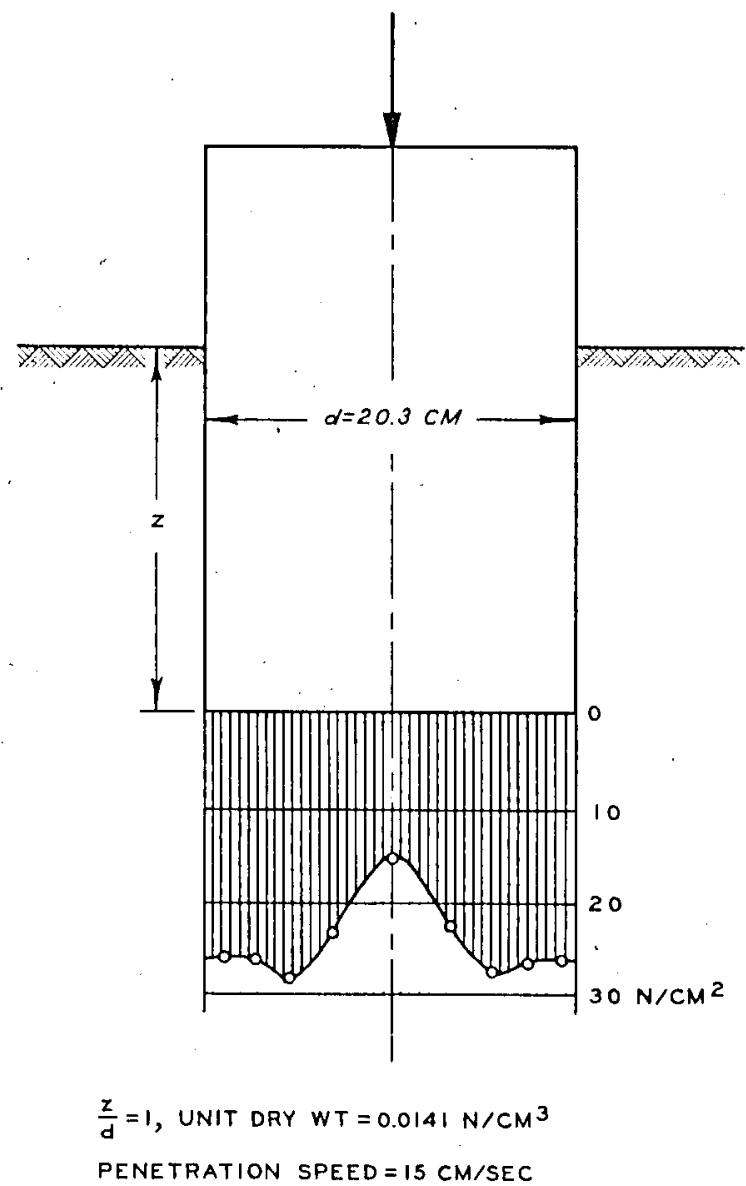

21. Thus, to evaluate the relative importance of the influence of rigidity variation on stress distribution beneath tires becomes very difficult. Numerous other factors probably have equal or greater effect on the stress distribution. On the other hand, the observed agreement between rigidity and stress distribution patterns for low-inflated tires can hardly be considered coincidence. A test program in which only the rigidity pattern is varied, with a]l other factors being constant, would clarify this question. But this ideal condition cannot be fulfilled, since a change in rigidity invariably affects the size and shape of the soilwheel interface, as illustrated in figs. 5 and 6 . 


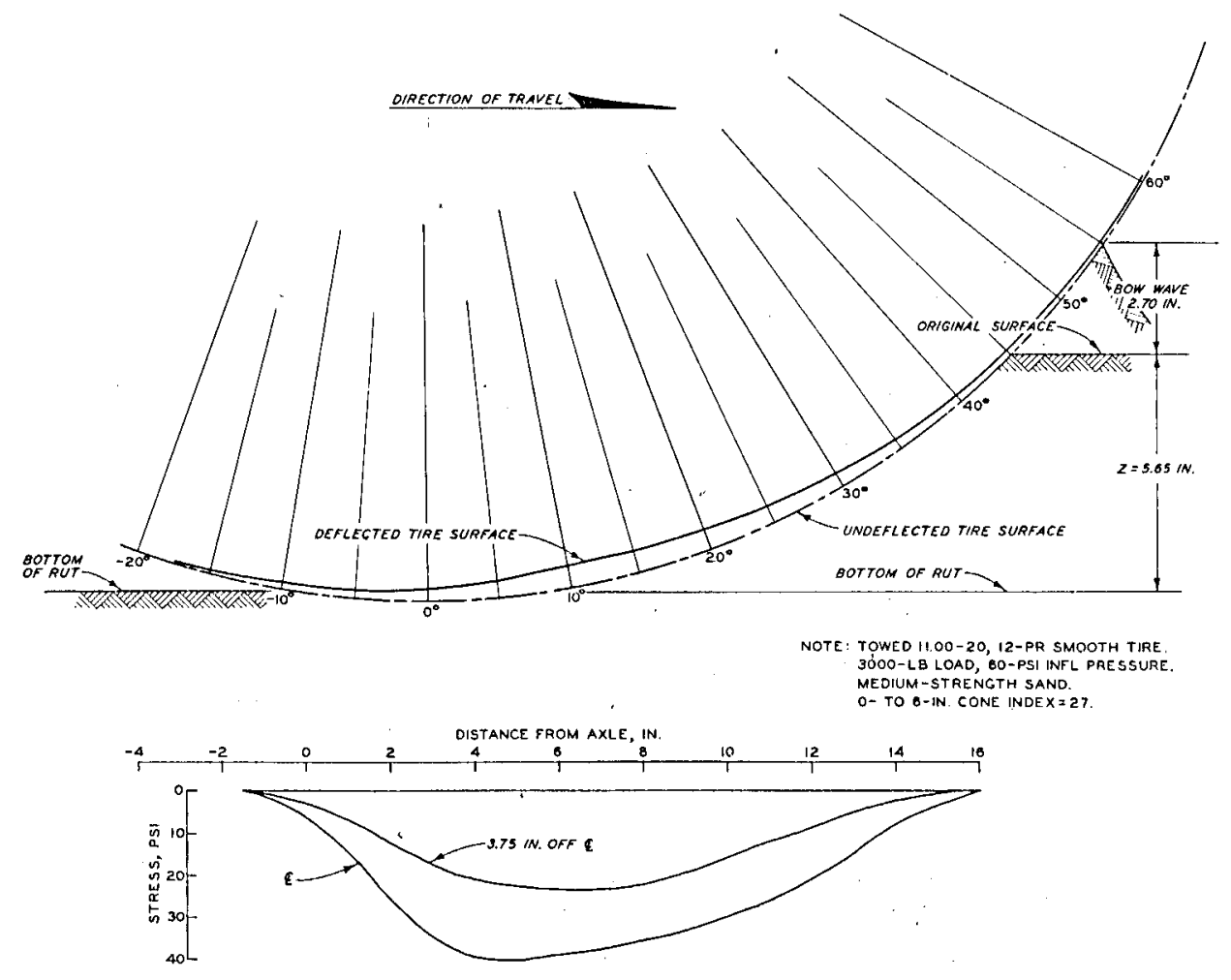

Fig. 5. Normal stresses on a tire surface; 60-psi tire inflation pressure, 0 - to 6-in. cone index $=27$ (from reference 2 )
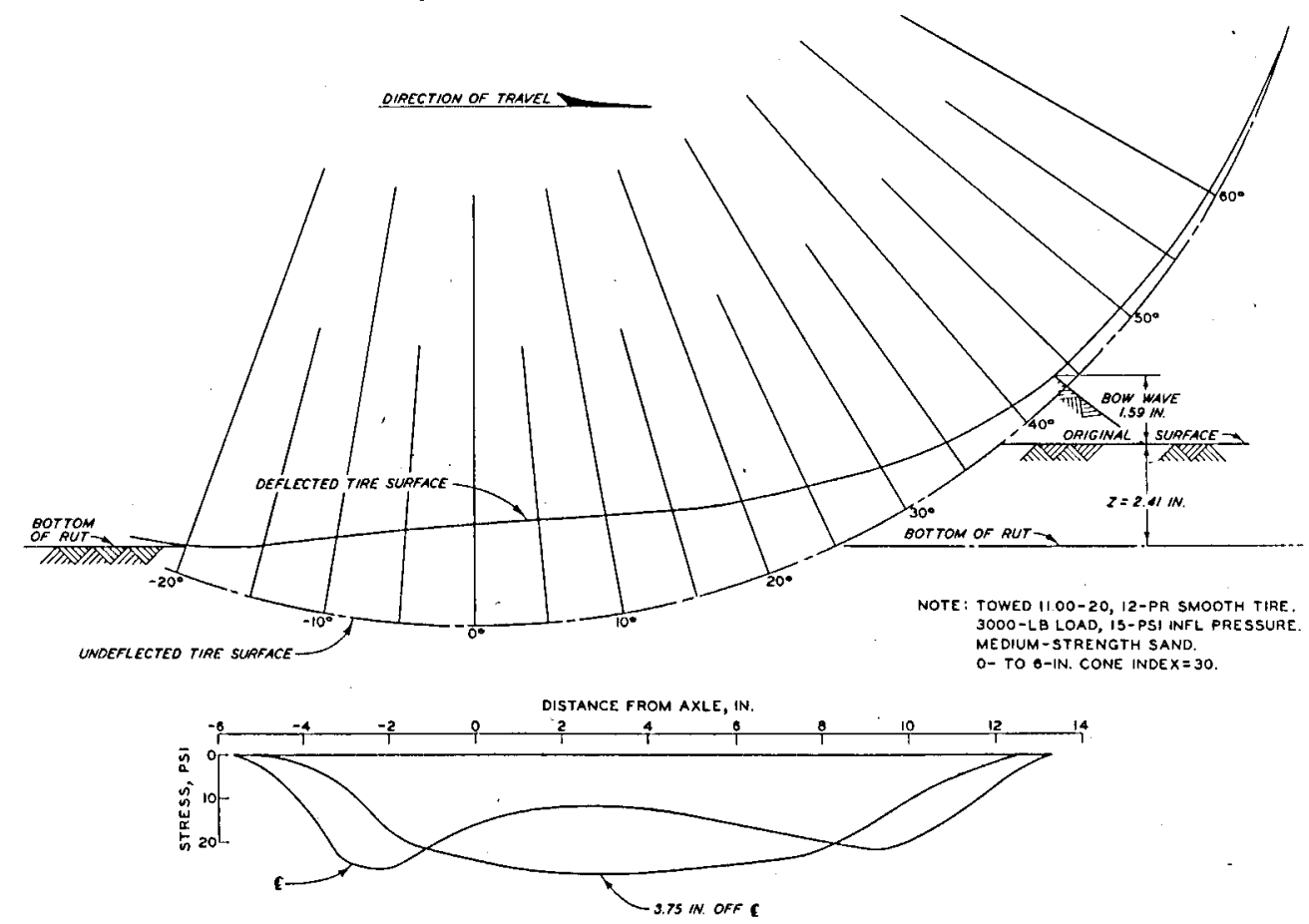

Fig. 6. Normal stresses on a tire surface; 15-psi tire inflation pressure, 0- to 6-in. cone index $=30$ (from reference 2) 
22. The stress distribution within a loose sand beneath a highly inflated tire is shown in fig. 5. This tire behaves almost like a rigid wheel, in that the deflection is rather small. Because of the high inflation pressure, the contribution of tire flexing and sidewall stiffness to the local rigidity is comparatively small with respect to the contribution of the inflation pressure, and the rigidity distribution can be assumed to be uniform. Accordingly, the stress distribution is of the one-peak type characteristic of wheels with uniform rigidity.

23. The pressure distribution beneath the same, but low-inflated, tire in practically identical test conditions is shown in fig. 6. The influence of the sidewall and flexing rigidity is significant (the flexing is also more pronounced than in fig. 5), and the maximum stresses are shifted from the central part of the interface to the peripheral areas of higher rigidity. But at the same time, the maximum tire deflection increases by four times, sinkage decreases more than half, and contact length, while remaining roughly unchanged, is shifted backward with respect to the bottom dead center. (compare figs. 5 and 6). To what extent these geometrical changes contribute to the reversal of the stress distribution pattern will remain an unanswered question until more is known about the internal mechanism of the soil-wheel interaction. The geometrical and mechanical facets of the problem are interrelated and cannot be clearly separated by experimental procedures.

24. As a conclusion of this discussion, nevertheless, the assumption appears justified that the rigidity distribution at the tire surface, for tires on sand, influences the stress distribution at the soil-wheel in.. terface to a significant, degree. A comparison of figs. 5 and 6 suggests that the relation between rigidity pattern and stress distribution be formulated, not directly between local rigidity and stress, but in reference to the standard stress distribution of a uniformly rigid wheel:

For wheels on sand, variations of local tire rigidity within the soil-wheel interface tend, within certain limitations, to shift the position of maximum normal stress from its standard location in the reference case of a uniformly rigid wheel toward the areas of higher local rigidity. 
The development of the experimental. wheel with controlled circumferential. rigidity, reported herein, was based upon this postulate.

\section{Numerical Evaluation}

25. The system of forces acting on a powered rigid wheel is represented in fig. 7. The soil reaction forces are the resultant $\mathrm{N}$ of the radial stresses and the resultant $T$ of the tangential stresses, acting at the soil-wheel interface. As can be seen in this schematic representation, a shift of $\mathbb{N}$ backwards to $\bar{N}$, as indicated, constitutes an improvement of

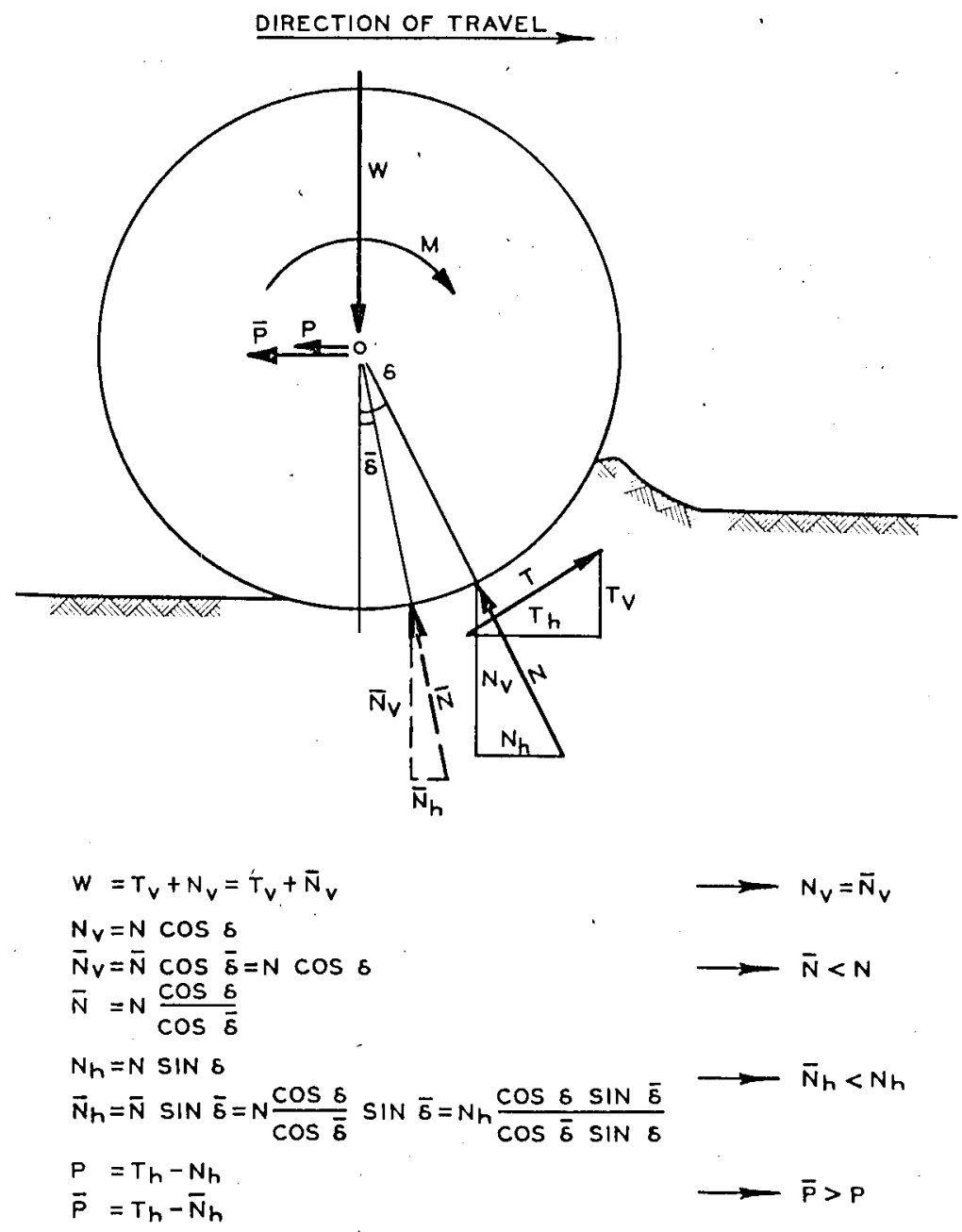

Fig. 7. Soil reaction resultants at the soil-wheel interface; powered rigid wheel. 
the soil reaction force system. If, for the sake of reasoning, the resultant $T$ is assumed unchanged by this operation, the shifted resultant $\bar{N}$ is smaller than $\mathbb{N}$, because $\bar{N}$ is more effective in carrying the vertical load $W$. The horizontal component of $\bar{N}$ (which is sometimes called motion resistance) is reduced with respect to that of $\mathbb{N}$ for two reasons: first, the absolute value of $\bar{N}$ is smaller than that of $N$; and.second, the inclination of $\bar{N}$ with respect to the vertical is smaller than that of $\mathbb{N}$. The result of the operation is an increase of available traction force, if all other quantities are unchanged. This" simple reasoning is presented here as a starting consideration. A more detailed numerical evaluation, presented next, shows the situation to be much more complex. In particular, the shifting of the resultant $\mathbb{N}$ is shown to affect other pertinent quantities, as might be expected.

26. According to the basic hypothesis stated in paragraph 24 , the position of the resultant $N$ is only. indirectly influenced by the rigidity distribution, the directly affected variable being the location of the maximum stress. For single-peak stress distributions, the shifting of the location of maximum stress is equivalent to the displacement in the same direction, but not for the same distance, of the resultant. The experimental wheel, described in Part III of this report, permitted the controi of local (circumferential) rigidity according to a preestablished pattern in order to shift the location of the maximum stress. For the theoretical evaluation of the results of tests with this wheel, the stress distribution at the interface throughout the test program was assumed to be of the. singlepeak type, such as found beneath highly inflated tires. This assumption is justified by the test program, since the rigidity distribution investigated possessed only one maximum value.*

27. To obtain an idea of the order of magnitude of the performance variations anticipated by shifting of the peak pressure location at the tire-wheel interface, the following numerical example is used: assume the average normal stress $(\sigma)$ along the contact length (average taken over

* Single peak refers here to the distribution along the center line of the tire. The distribution over the width of the tire is not relevant to the wheel performance and was not directly controlled in the test program. 
width $B$ of the rigid wheel) follows the equation (fig. 3):

Zone I: $\quad(-\beta<\delta<\lambda \alpha): \sigma=\sigma_{m} \sin \frac{\pi}{2} \cdot \frac{\delta+\beta}{\lambda \alpha+\beta}$

Zone II: $\cdot(\lambda \alpha<\delta<\alpha): \sigma=\sigma_{m} \cos \frac{\pi}{2} \frac{\delta-\lambda \alpha}{\alpha-\lambda \alpha}$

The above equations are distorted sine and cosine functions, which meet at the point $(\lambda \alpha)$ of maximum stress $\left(\sigma_{m}\right)$. Thi's type of stress distribution is similar to that for highly inflated tires or rigid wheels.

28. For simplicity, assume further an undeforming wheel and a constant mean $\frac{T}{\sigma}=t$ ratio along the soil-tire interface, with $T$ being the shear stress. Load (W), pull (P), torque (M), and efficiency (E) are then given by:

$$
\begin{gathered}
W=R B \int_{-\beta}^{\alpha} \sigma \cos \delta d \delta+\int_{-\beta}^{\alpha} t \sigma \sin \delta d \delta \\
P=R B \int_{-\beta}^{\alpha} \sigma \cos \delta d \delta-\int_{-\beta}^{\alpha} t \sigma \sin \delta d \delta \\
M=R^{2} B \int_{-\beta}^{\alpha} t \sigma d \delta \\
E=\frac{P V}{M \omega}=\frac{P R(1-s)}{M}
\end{gathered}
$$

The efficiency $(\mathrm{E})$, as proposed by Leflaive, ${ }^{6}$ is the ratio of energy output (pull $\times$ translational velocity) over energy input (torque $\times$ rotational velocity) of the wheel as a traction device. Equation 5 proved very useful in the interpretation of the test results.

29. With the abbreviations:

$$
\mathrm{b}=\frac{\pi}{2(\beta+\lambda \alpha)} \quad \text { and } \quad \mathrm{c}=\frac{\pi}{2 \alpha(1-\lambda)}
$$


equations 1-4 yield, after integration and rearrangement:

$$
\begin{aligned}
\frac{\mathrm{W}}{\mathrm{RB} \sigma_{\mathrm{m}}} & =\frac{1}{\mathrm{~b}^{2}-1}(\mathrm{~b} \cos \beta-\sin \lambda \alpha-\mathrm{tb} \sin \beta+\mathrm{t} \cos \lambda \alpha) \\
& +\frac{1}{\mathrm{c}^{2}-1}(\mathrm{c} \cos \alpha+\sin \lambda \alpha+t c \sin \alpha-t \cos \lambda \alpha) \\
\frac{\mathrm{P}}{\mathrm{RB} \sigma_{\mathrm{m}}} & =\frac{1}{\mathrm{~b}^{2}-1}(\mathrm{tb} \cos \beta-\mathrm{t} \sin \lambda \alpha+\mathrm{b} \sin \beta-\cos \lambda \alpha) \\
& +\frac{1}{\mathrm{c}^{2}-1}(\mathrm{tc} \cos \alpha+\mathrm{t} \sin \lambda \alpha-\mathrm{c} \sin \alpha+\cos \lambda \alpha) \\
\frac{\mathrm{M}}{\mathrm{R}^{2} \mathrm{~B} \sigma_{\mathrm{m}}}=t\left(\frac{1}{\mathrm{c}}+\frac{1}{\mathrm{~b}}\right)=\frac{2 t}{\pi}(\alpha+\beta) &
\end{aligned}
$$

As an example, take the numerical values:*

$$
\begin{aligned}
t & =0.4 \\
\beta & =0.2 \alpha \\
\mathrm{W} & =4000 \mathrm{~N} \\
\mathrm{R} & =35 \mathrm{~cm} \\
\mathrm{~B} & =12 \mathrm{~cm} \\
\sigma_{\mathrm{m}} & =20 \mathrm{~N} / \mathrm{cm}^{2} \\
\mathrm{~W} / \mathrm{RB} \sigma_{\mathrm{m}} & =0.476
\end{aligned}
$$

Further, let $\lambda$, which indicates the relative position of the maximum stress with respect to $\alpha$, be the variable. The lower the value of $\lambda$, the more the resultant of the stresses is shifted rearward.

30. Since the wheel is supposed to carry the same vertical load re- . gardless of the value of $\lambda$, the upper integration limit, $\alpha$, has first to be determined as a function of $\dot{\lambda}$. In equation 6 , the value of $\alpha$ has to

* These values are approximately equal to the conditions in the experimental wheel tests. An exact agreement was not attempted for this rough evaluation nor was it possible, since the exact values of $R$ and $\sigma_{m}$ were unknown. Values for $\beta$ and $\sigma_{m}$ were assumed and are considered reasonable, based on previous Waterways Experiment station experience. 
be chosen so that the right-hand part of equation 6 is equal to $\mathrm{W} / \mathrm{RBO}_{\mathrm{m}}=0.476$ for every value of $\lambda$. This has to be done by trial, since equation 6 cannot be solved for $\alpha$ in an explicit form. The results are given in the following table:

Table 1

\begin{tabular}{clllll}
\hline$\lambda$ & 0.8 & 0.6 & 0.4 & 0.2 & 0.0 \\
$\alpha$ (radians) & 0.588 & 0.59 & 0.592 & 0.596 & 0.602 \\
\hline
\end{tabular}

31. This result is rather astonishing because one would expect the contact length (and with that the sinkage) to become smaller when the location of the maximum pressure approaches the vertical reference position, since the radial pressures then become more efficient in carrying the vertical load. It turns out, however, that for the chosen numerical example, the load-carrying fraction of the tangential stresses is relatively high (about 11 percent for $\lambda=0.8$ ). With decreasing $\lambda$ values, the loadcarrying capacity of tangential stresses diminishes more than that of the radial stresses increases, so that the contact length (and hence sinkage) has to increase in order to provide the full load-carrying capacity.

32. When the values of table I, together with the other chosen numerical values, are introduced into equations 5,7 , and 8 , the following results are obtained:

Table 2

\begin{tabular}{|c|c|c|c|c|}
\hline$\lambda$ & $\begin{array}{l}\mathrm{Pull} \\
\frac{\mathrm{P}}{\mathrm{RBO} \sigma_{\mathrm{m}}} \\
\end{array}$ & $\begin{array}{l}\text { Torque } \\
\frac{\mathrm{M}}{\mathrm{R}^{2} \mathrm{~B} \sigma_{\mathrm{m}}} \\
\end{array}$ & $\begin{array}{l}\text { Efficiency } \\
\frac{E}{(I-s)}\end{array}$ & $\begin{array}{c}\text { Sinkage } \\
\frac{\mathrm{z}}{\mathrm{R}}=1-\cos \alpha\end{array}$ \\
\hline 0.8 & 0.0384 & 0.1799 & 0.213 & 0.168 \\
\hline 0.6 & 0.0539 & 0.1803 & 0.299 & 0.169 \\
\hline 0.4 & 0.0689 & 0.1811 & 0.380 & 0.170 \\
\hline 0.2 & 0.0843 & 0.1824 & 0.461 & 0.172 \\
\hline 0.0 & 0.1008 & 0.1841 & 0.546 & 0.176 \\
\hline
\end{tabular}


33. The stress distribution for $\lambda=0.4$, which represents the symmetrical case with respect to the total contact angle $(\alpha+\beta)$, is taken as the reference distribution. From table 2, a shift of the location $\lambda$ of the maximum stress backwards from $\lambda=0.4$ to $\lambda=0.2$ appears to increase the traction capability $P$ of the wheel by 22.4 percent, despite the fact that the sinkage increases by 1.2 percent. The torque remains approximately constant, and the increase of efficiency, $E$, by 21.3 percent (assuming the slip is unchanged) is due practically to the pull increase alone. The unfavorable shift forward to $\lambda^{\prime \prime}=0.6$ causes pulz and efficiency to decrease by roughly the same percentages as those above for the favorable shift.

34. The example shows that a variation of the normal pressure distribution, which can by no means be called a dramatic change (see fig. 8), results in a substantial performarce increase. This occurs because the resultant of the normal stress is shifted into a more favorable position, and the resultant of the tangential stress becomes more horizontally oriented and thus more effective, since normal and tangential stresses are linked for this numerical example by the expression $T=t \sigma$.

35. The increase in efficiency has been evaluated with constant slip assumed. On the other hand, slip and the location of maximum radial stress are known to be interrelated. 7 A decrease in slip causes a decrease in $\lambda$. Therefore, the reverse might be antic-

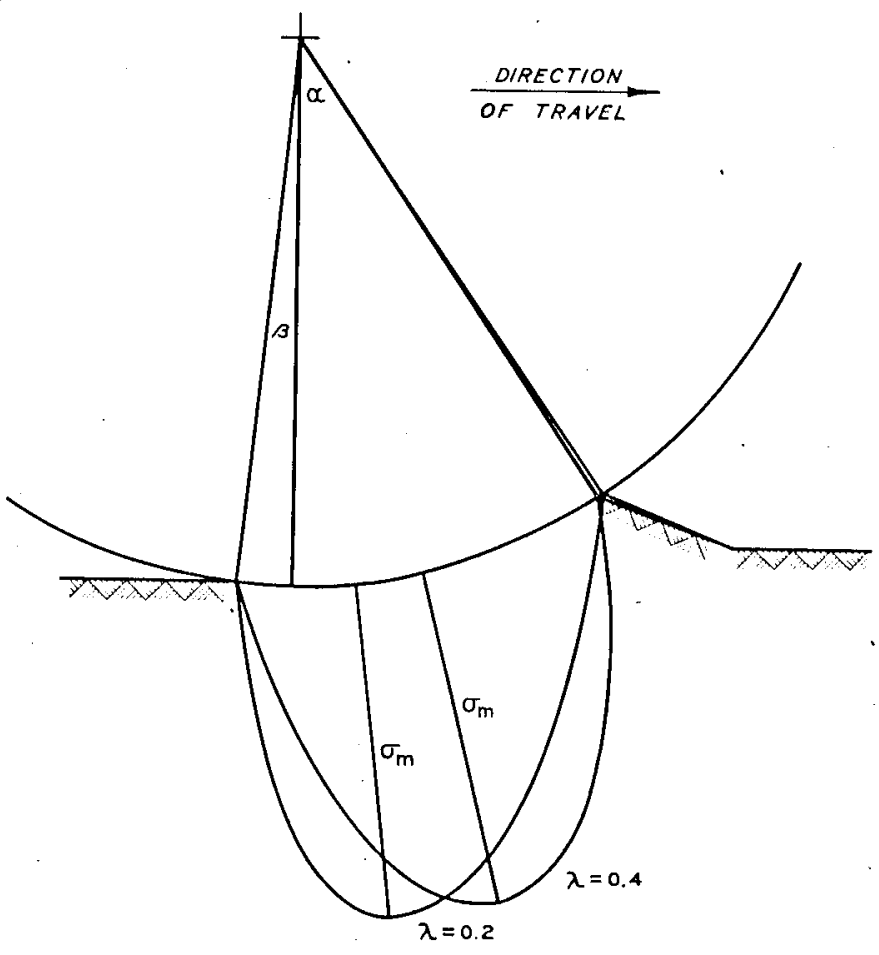
ipated, i.e. a decrease of $\lambda$ by direct interference

Fig. 8. Radial stress distribution for $\lambda=0.2$ and $\lambda=0.4$ 
with the stress distribution results in a decrease of slip. If, for the purpose of demonstration, the slip in the reference case $(\lambda=0.4)$ is assumed to be 20 percent, and for $\lambda=0.2$ to be 15 percent, the increase in efficiency would be

$$
\frac{0.461(1-0.15)-0.380 \cdot(1-0.2)}{0.380(1-0.2)} \cdot 100=29 \text { percent }
$$

instead of 21.3 percent as previously obtained.

36. In any case, a conclusion can be drawn from the numerical example presented that a direct interference with the pressure distribution is a worthwhile goal to be pursued. When the position of the peak pressure can be shifted backward, the wheel not only develops higher traction capability, but does so at an increased efficiency.

37. However, as might be expected, the performance improvements are less significant when the soil strength is high. (For an unyielding surface, the effect would be practically zero.) This point is illustrated by the results of a numerical example that differs from the previous one only in that the values $\sigma_{m}$ and $t$, which are characteristic for the soil strength, have been increased by 50 percent:

$$
\begin{array}{rlrl}
\mathrm{t} & =0.6 & \mathrm{~B} & =12 \mathrm{~cm} \\
\beta & =0.2 \alpha & \sigma_{\mathrm{m}} & =30 \mathrm{~N} / \mathrm{cm}^{2} \\
\mathrm{~W} & =4000 \mathrm{~N} & \mathrm{~W} / \mathrm{RBO}_{\mathrm{m}} & =0.318 \\
\mathrm{R} & =35 \mathrm{~cm} &
\end{array}
$$

\begin{tabular}{|c|c|c|c|c|c|}
\hline$\lambda$ & $\alpha$ & $\begin{array}{l}\mathrm{Pull} \\
\frac{\mathrm{P}}{\mathrm{RB} \sigma_{\mathrm{m}}}\end{array}$ & $\begin{array}{l}\text { Torque } \\
\frac{\mathrm{M}}{\mathrm{R}^{2} \mathrm{~B} \sigma_{\mathrm{m}}} \\
\end{array}$ & $\begin{array}{c}\text { Efficiency } \\
\frac{E}{(1-s)} \\
\end{array}$ & $\begin{array}{c}\text { Sinkage } \\
\frac{\mathrm{z}}{\mathrm{R}}=1-\cos \alpha \\
\end{array}$ \\
\hline 0.6 & 0.382 & 0.1217 & 0.175 & 0.695 & 0.072 \\
\hline 0.4 & 0.386 & 0.1289 & 0.177 & 0.728 & 0.073 \\
\hline 0.2 & 0.391 & 0.1364 & 0.179 & 0.763 & 0.075 \\
\hline
\end{tabular}

The numerical results from equations 5-8, as used previously, are summarized in table 3 :

Table 3 
38. Although the value for soil strength has been multiplied by a factor of only 1.5, the performance increase is small relative to the increase determined at the lesser strength. The actual value of the pull increase is only one-fourth as great ( 5.8 percent pull increase as compared with 22.4 percent) when $\lambda$ is shifted from 0.4 to 0.2 . The direct interference with the stress distribution rapidly loses its advantages as soil conditions improve.

39. At the other extreme, the experimental wheel might be ineffective in soft soils, too, because very weak soils might be unable to sustain any variation of the pressure distribution pattern, as explained in paragraphs 18 and 19. Thus, the principle of the experimental wheel probably will apply neither in very weak nor on very firm soils. These limitations leave room for possible improvements in the range of fair to poor terrain conditions, where improvements are most needed. 
PART III: DESCRIPTION OF THE EXPERIMEIVTAL WHEEL

AND TEST PROCEDURES

\section{Experimental Wheel}

Mechanical system

40. The system adopted to achieve local control of tire rigidity is represented schematically in fig. 9. It consists basically of six hydraulic jacks arranged inside the wheel in a roughly radial manner. The ends of the pistons are attached to a sliding shoe lying on the inner side of the tire's rolling surface. The upper ends of the jacks and of the shoe are attached to a nonrotating body, so that the whole jack-shoe system remains in a fixed position with the tire sliding against the shoe. All mechanical connections of the system. are articulated so that the system can follow freely the various possible tire deformations. Views of the internal system of the experimental wheel are shown in fig. 10 .

41. Each hydraulic jack can be made to exert a certain force through the piston to the sliding shoe. These single forces are then spread by the sliding shoe and converted into stresses acting with the inflation pressure against the inner side of the tire's rolling surface. The higher these internal stresses are, the less the tire deforms under the action of external. soil reaction forces, $i . e$. the more rigidly the tire behaves.

42. The critical element within the system is the sliding shoe, which has to exhibit a high flexural stiffness to spread the single piston forces, but which also has to be rather flexible to adapt to the various geometrical configurations of the tire. Finally, the shoe has to present a very smooth outer surface to reduce friction with the rotating tire. The solution to this problem of conflicting requirements was solved in the following way: the shoe consists of a rather firm rubber strip $(2.5 \mathrm{~cm}$ thick, $8 \mathrm{~cm}$ wide, and $60 \mathrm{~cm}$ long) tightly wrapped in a spiral fashion by a polished steel band approximately $2 \mathrm{~cm}$ wide with roughly $1 \mathrm{~cm}$ of overlap. In. this manner, the shoe exhibits high resistance against locally confined bending, and thus assures a good load-spreading capability both in the traverse and in the longitudinal directions. On the other hand, moderately 


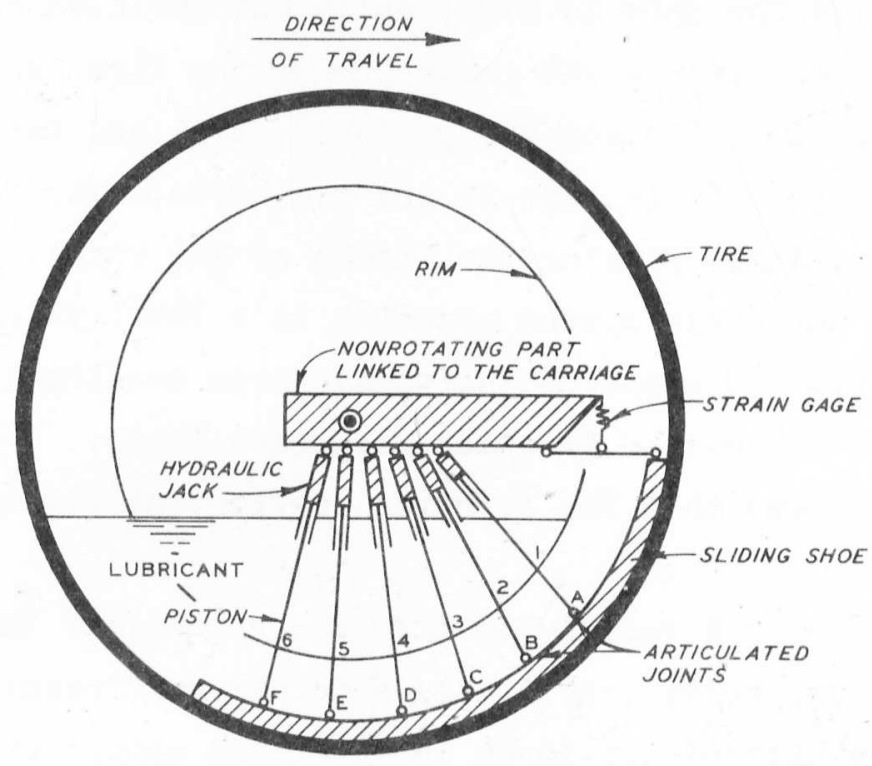

Fig. 9. Schema of the mechanical system of the experimental wheel
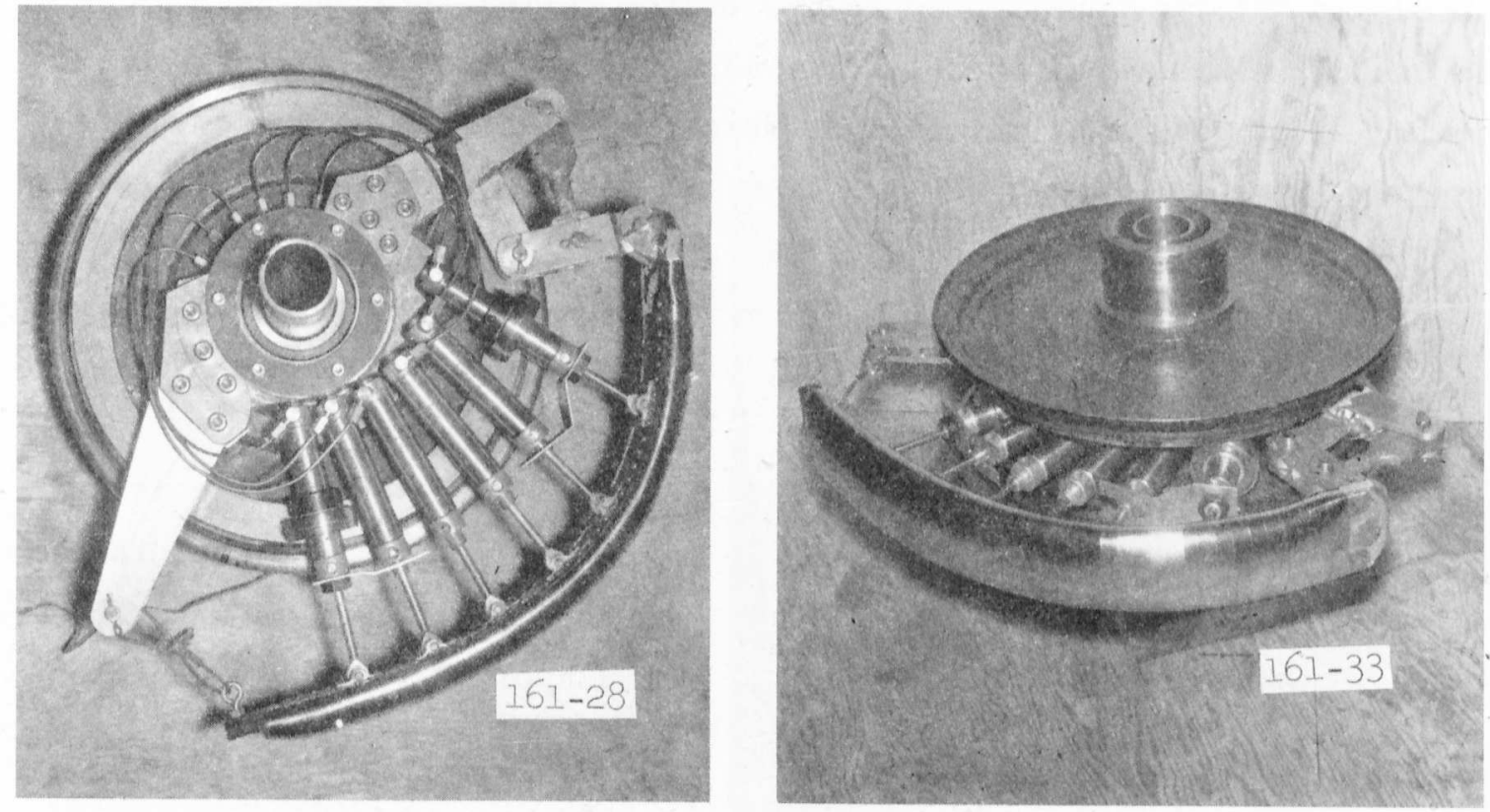

Fig. 10. Views of internal system of experimental wheel 
long wave bending of the shoe is practically unrestricted by this construction, and the shoe can follow the curvature of the tire as required. Also, the friction coefficient between the polished steel and the rubber of the smoothed inner surface of the tire is not excessive. The friction was further reduced by partially filling the inside of the wheel with a liquid lubricant so that the whole system operates in a lubricating bath (fig. 9). The strain gage (fig. 9) measures the total force developed in the shoe, but only part of it is due to friction, as shown later. The analysis of the test results showed that the friction coefficient was approximately $0.10-0.15$.

43. The Michelin X radial-ply tire was chosen for the tests. This low-pressure tire has relatively little sidewall stiffness; therefore, it does not interfere excessively in an uncontrolled manner with the preestablished pattern of rigidity distribution. In addition to this advantage, radial-ply tires feature a strong rolling belt, which further increases the load-spreading capability of the system. The tread pattern was buffed smooth; the undeflected radius of the buffed tire was $34.6 \mathrm{~cm}$, and the overall width was $16.5 \mathrm{~cm}$. Fig. 11 shows the assembled experimental wheel. It is driven by the chains, which are connected to the driving

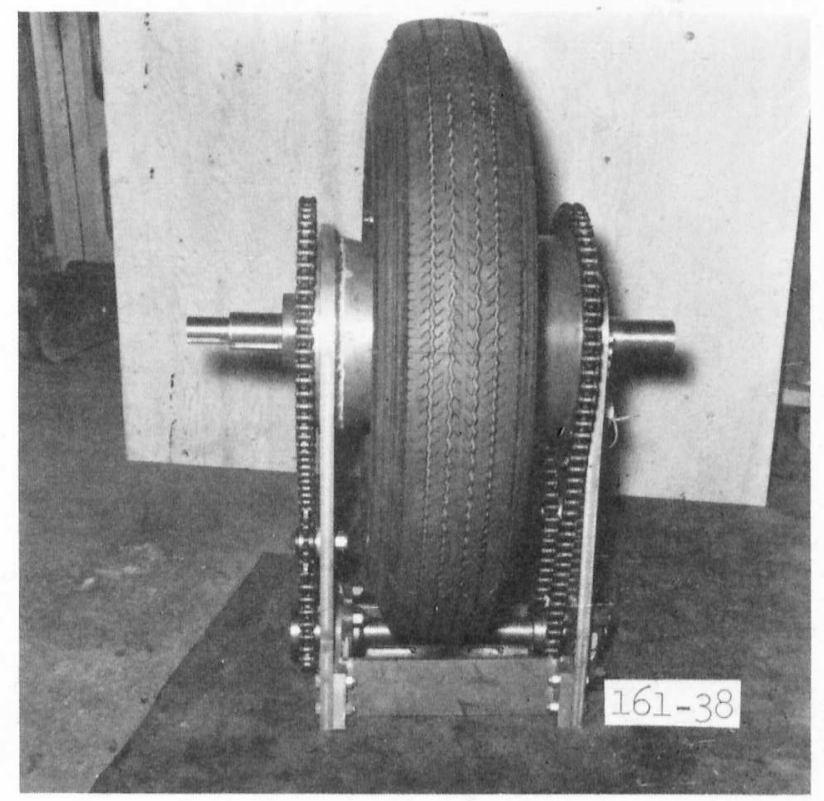

Fig. 11. Assembled experimental wheel shaft of the carriage; the nonrotating internal system is linked to the outer frame, which is fastened to the carriage. Hydraulic system

44. A schematic design of the hydraulic system is presented in fig. 12. Air pressure is applied to the accumulator and transmitted through a membrane to the hydraulic fluid, the pressure of which is monitored by a pressure gage. The accumulator is connected to the master cylinder inside the 


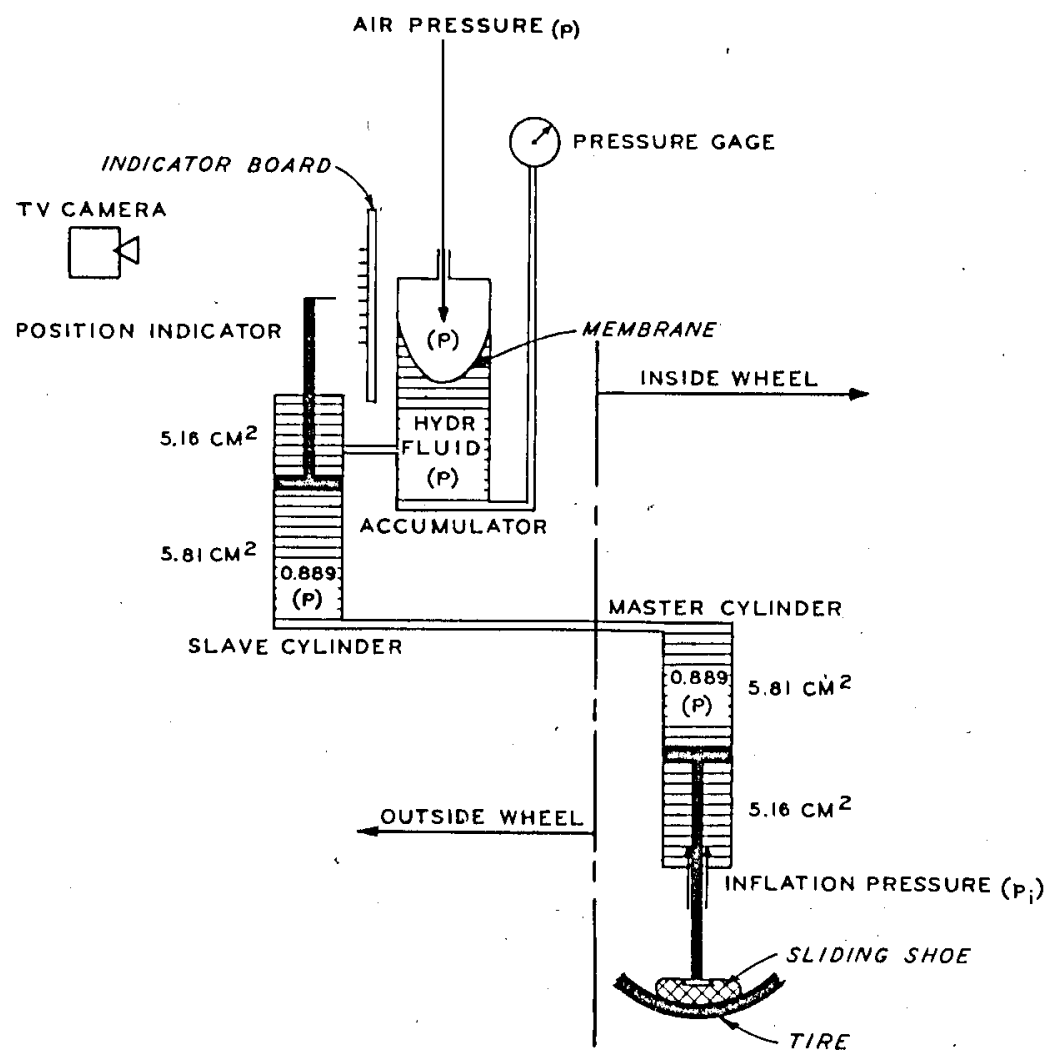

Fig. 12. Schema of the hydraulic system of the experimental wheel

wheel, with a slave cylinder being intercalated. The slave cylinder has the same dimensions as the master cylinder, its purpose being to indicate the position of the piston of the master cylinder inside the wheel. After calibration of the system, which has to be done prior to the wheel assemblage, the position of the master cylinder piston can be read on the indi-. cator board. When the position of each piston is known, the geometry along the center line of the soil-wheel interface and the static system of the internal forces can be determined.

45. Because the rod section is $0.65 \mathrm{~cm}^{2}$ in area, the free area of the piston at the rod side is less than the area at the other side (5.16 versus $5.81 \mathrm{~cm}^{2}$, fig. 12). Accordingly, the pressure in the master and slave cylinders is 0.889 times the pressure in the accumulator. The actual force transmitted to the sliding shoe is further reduced by the inflation pressure of the tire, which acts against the fluid pressure of the master 
cylinder inside the tire. With $p$ being the applied accumulator pressure and $p_{i}$ the tire inflation pressure, the force $(F)$ in the rod of the master cylinder is

$$
F(N)=0.889 p\left(N / \mathrm{cm}^{2}\right) \times 5.81\left(\mathrm{~cm}^{2}\right)-p_{i}\left(\mathbb{N} / \mathrm{cm}^{2}\right) \times 5.16\left(\mathrm{~cm}^{2}\right)
$$

46. Immediately prior to a test, the accumulators were charged, according to a preestablished schedule, with pressure up to $150 \mathrm{~N} / \mathrm{cm}^{2}$. A high-pressure nitrogen bottle was used, but during the testing, the accumlators were disconnected from the bottle. As a result, the cylinder pressures and piston positions varied during the testing, with the major variation occurring during a short starting period. The initial setting was irrelevant to the actual test conditions, so continuous recording of both piston displacements and pressure variation during the test was necessary. Mounted at the carriage was a tape-recording television camera that monitored the piston positions and the pressures as indicated on the indicator board (fig. 12).

\section{Static system}

47. The piston forces as computed from equation 9 cannot be treated directly in terms of local rigidity. Firstly, these piston forces might have a considerable tangential component, due to the nonradial arrangement of the hydraulic jacks (see fig. 9) as well as to the noncircular shape of the deformed tire. Only the normal components of these local internal forces are of concern, and their determination necessitates the exact knowledge of the jack-tire configuration. This problem is further discussed in Part IV of this' report. For demonstration, a circular tire shape is assumed here.

48. Secondly, the piston forces $\left(P_{i}\right)$ are not the only mechanical forces acting on the individual joints (labeled $A-F$ in fig. 9). The other forces involved are (fig. 13):

$$
\begin{aligned}
& \text { a. Tensile forces }\left(S_{i}\right) \text { of the sliding shoe. } \\
& \text { b. Tire-shoe friction forces }\left(F_{i}\right) \text {. } \\
& \text { c. Resulting tire reaction forces }\left(R_{i}\right) \text {. }
\end{aligned}
$$

The forces $F_{i}$ and $R_{i}$ are actually resultants of the shear $\left(\tau_{f}\right)$ and 


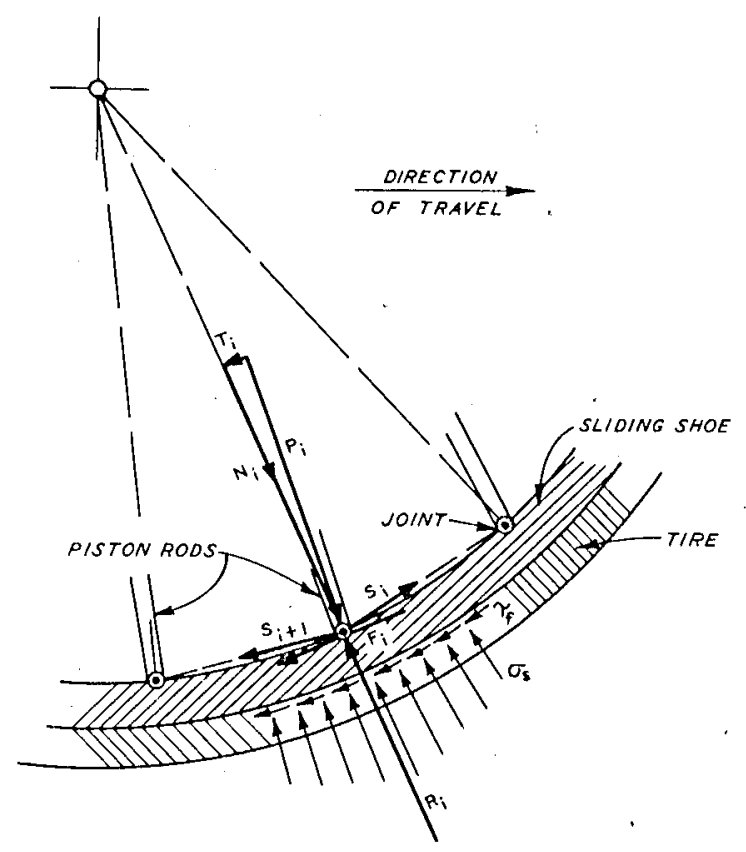

a. FORCES ACTING AT JUINT i

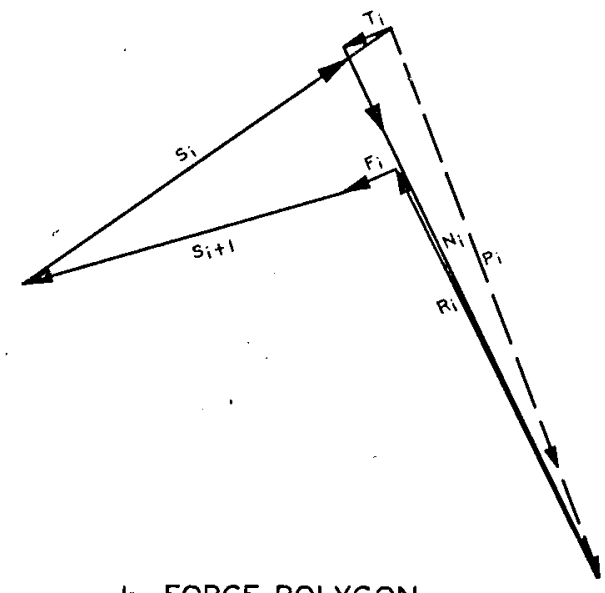

b. FORCE POLYGON

Fig. 13. Forces acting at articulated joint of experimental wheel

normal stresses $\left(\sigma_{S}\right)$ at the shoe-tire interface as shown in fig. 13. For simplicity, all forces are assumed to act directly at the joint. Because of the free articulation of these joints, the orientation of the tensile forces, $S_{i}$, is known to pass through the neighboring joints. Therefore, if the geometric conditions are known in the neighborhood of a given joint, the orientation of all forces acting on that joint are known. However, except for $P_{i}$, which is known from equation 9 and which can be decomposed into its normal and tangential components, $N_{i}$ and $T_{i}$, the magnitudes of. the four remaining forces are unknown and must be determined from the equilibrium conditions at the joint.

49. Principles of graphic statics were used so that the equilibrium condition could be represented by a closed polygon of the forces involved, such as shown in fig. 13b. $R_{i}$ is the force of concern that acts through the sliding shoe upon the inner surface of the tire and thus contributes to the local rigidity. Also, $R_{i}$, and not the force $P_{i}$, determines the friction at the sliding shoe; therefore, the friction force $F_{i}$ is known to be 


$$
F_{i}=k_{s} R_{i}
$$

with $k_{s}$ the coefficient of friction between rubber and polished steel. 50. Relation 10 reduces the number of unknown forces to three-$S_{i}, S_{i+1}$, and $R_{i}$. However, the problem is solvable only for two unknown forces, and the solution presented in fig. 13b is therefore not unique. It was actually based on the arbitrary decision on the length of one vector, for example, $S_{i}$. On the other hand, the whole system is in a difinite equilibrium. The $R_{i}$ forces can be determined only by a consideration of the whole system (fig. 14) rather than by consideration of the individual joints separately.

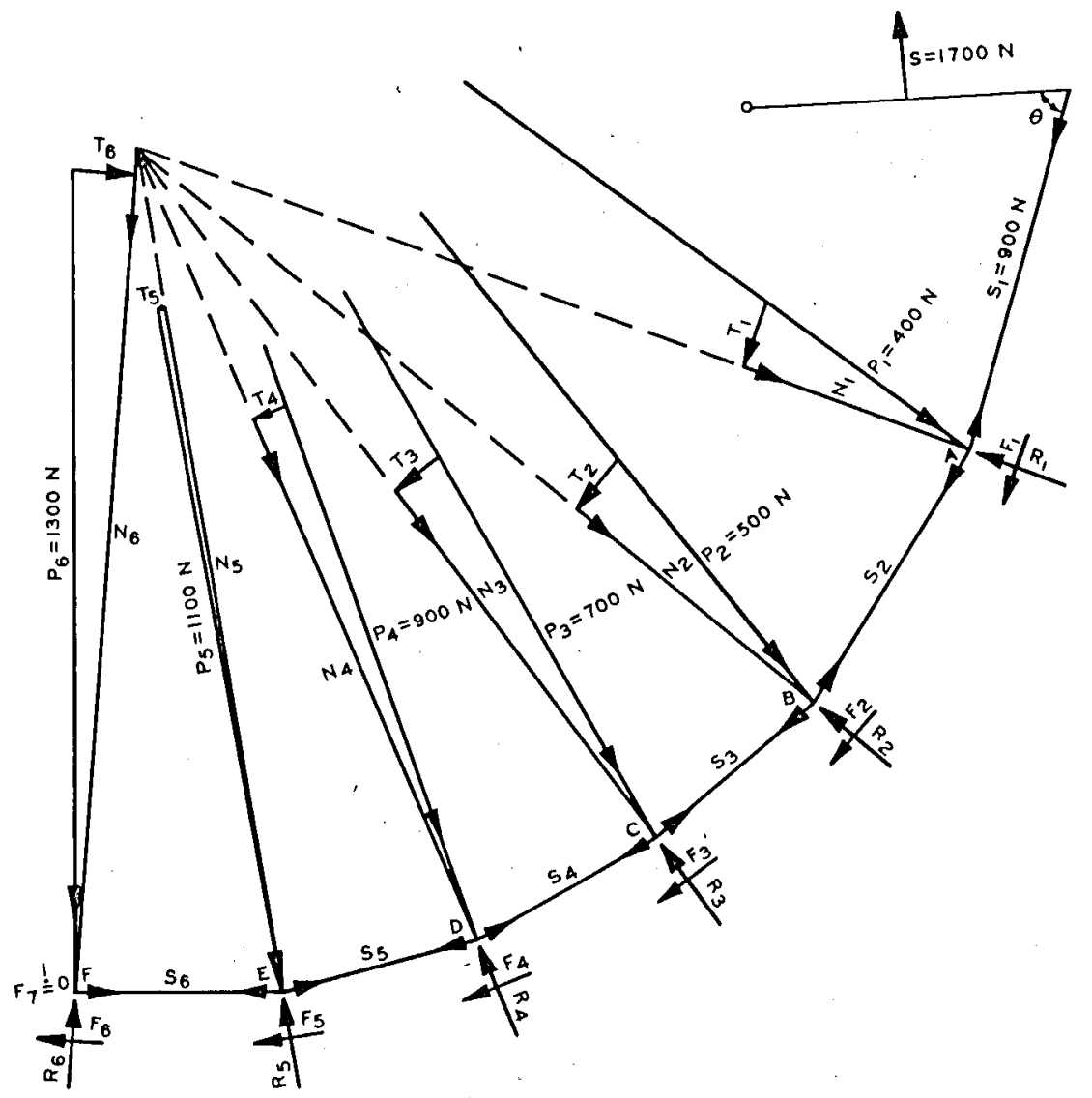

Fig. 14. Schema of the static system of the experimental wheel 
51. In fact, for the two particular joints $A$ and $F$ in $f i g .14$ the third force is known. For the joint $A$, the force $S_{1}$ is measured by the strain gage reading $\mathrm{S}$ :

$$
S_{1}=\frac{S}{2 \sin \theta}
$$

and for the joint $F$, the tensile force $S_{7}=0$. The unknown forces $\mathrm{R}_{1}, \mathrm{~F}_{1}$, and $\mathrm{S}_{2}$ can be determined by graphic construction, according to fig. 13b, using relation 10 with an estimated friction coefficient $k_{s}$. The force $\mathrm{S}_{2}$ then is known, the vector polygon for the joint $B$ can be drawn, and the same procedures can be used from joint to joint. The soIution depends on the correct estimate of $\mathrm{k}_{\mathrm{S}}$, which is controlled by the condition that no rest force should remain for the joint $F$. In other words, the correct solution is obtained if the force $\mathrm{S}_{7}$ (which represents the tensionless free end of the sliding shoe) equals zero. Otherwise, the procedure has to be repeated with an improved estimate of the friction coefficient $k_{s}$.

52. By using the forces $S_{i}$ drawn from the previous step for the next step, i.e. by linking the individual vector polygons, the graphic solution for the case given in fig. 14 is shown in fig. 15. For $\mathrm{k}_{\mathrm{s}}=0.10$, there remains a positive tensile force $S_{7}$ of $280 \mathrm{~N}$ ( $f i g$. 15a), which is reduced to $\mathrm{S}_{7}=50 \mathrm{~N}$ by using $\mathrm{k}_{\mathrm{s}}=0.15$ (fig. 15b). A friction coefficient of $k_{s} \approx 0.16$ would give the correct solution with $S_{7}=0$.

53. A comparison of the solutions (figs. 15a and 15b) indicates, however, that the magnitude of the forces $R_{i}$ are not too sensibly affected. by a variation of $k_{s}$. There is no necessity, therefore, to push the exactitude of the graphic construction too far, and a simplified method could be devised, consisting of handling force $T_{i}$ as parallel to $S_{i}$ and force $F_{i}$ as parallel to $S_{i+1}$. This considerably reduces the complexity of the graphic solution, as shown in fig. 16. The error in forces $R_{i}$ involved by using the simplified method is only about 2 percent (compare fig. 16a with fig. 15b). A detailed description of the procedures used in the simplified graphic solution is given in Appendix A. 


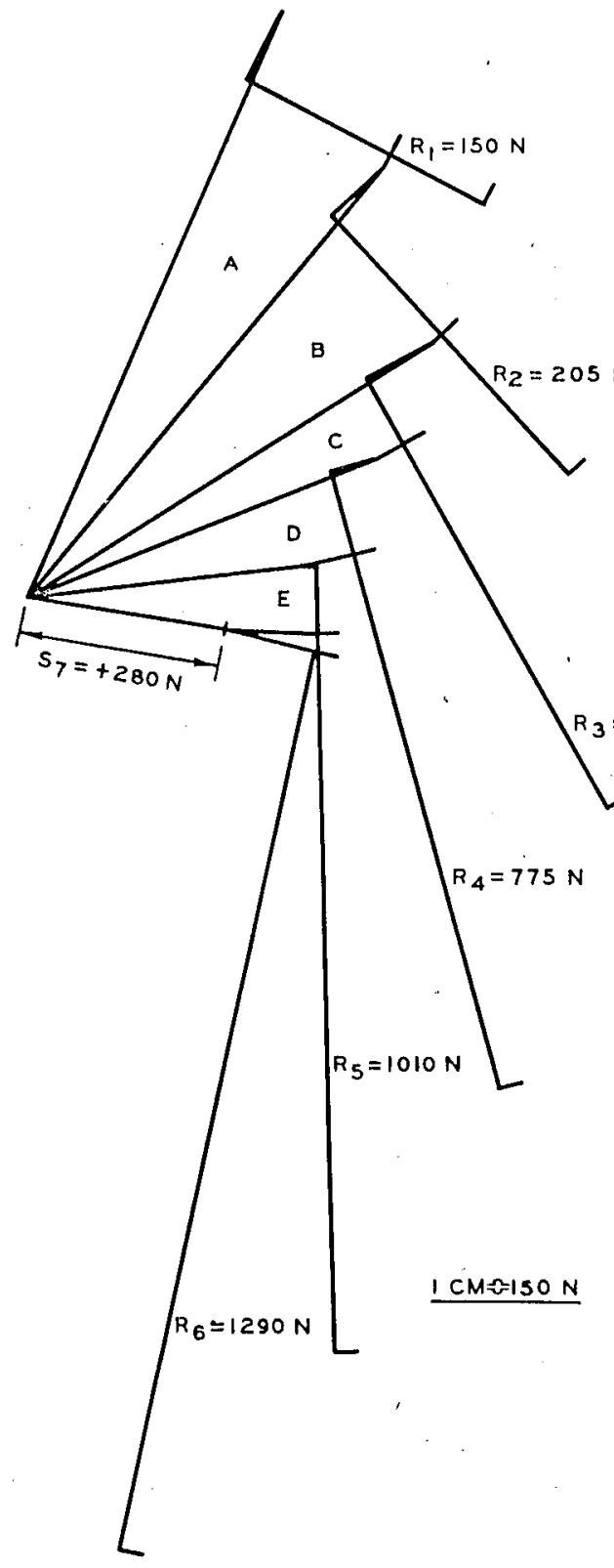

a. $10 \%$ FRICTION

$k_{S}=0.10$

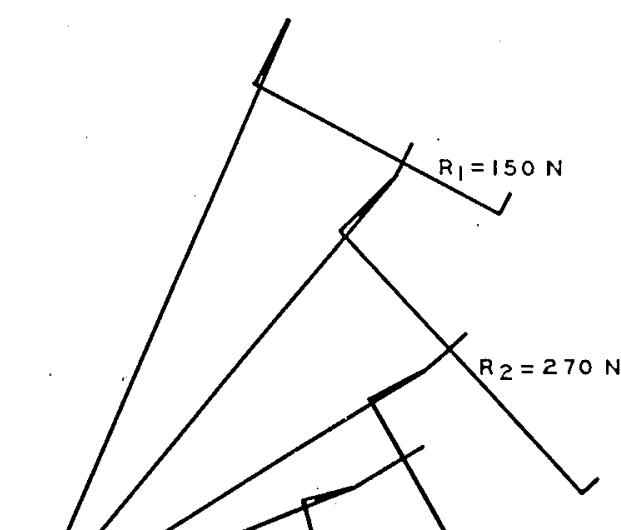

$\sum_{R_{4}=800 \mathrm{~N}}$

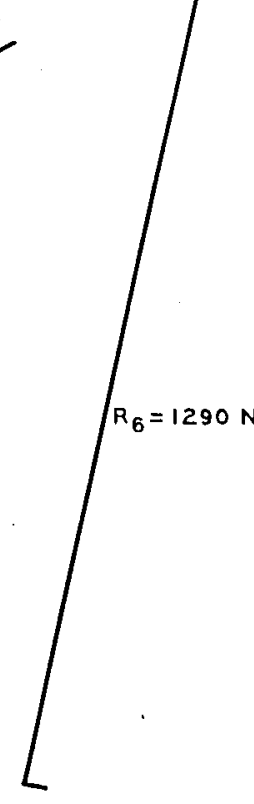

b. $15 \%$ FRICTION

$\mathrm{K}_{\mathrm{S}}=0.15$

Fig. 15. Graphic solution of the internal force system 


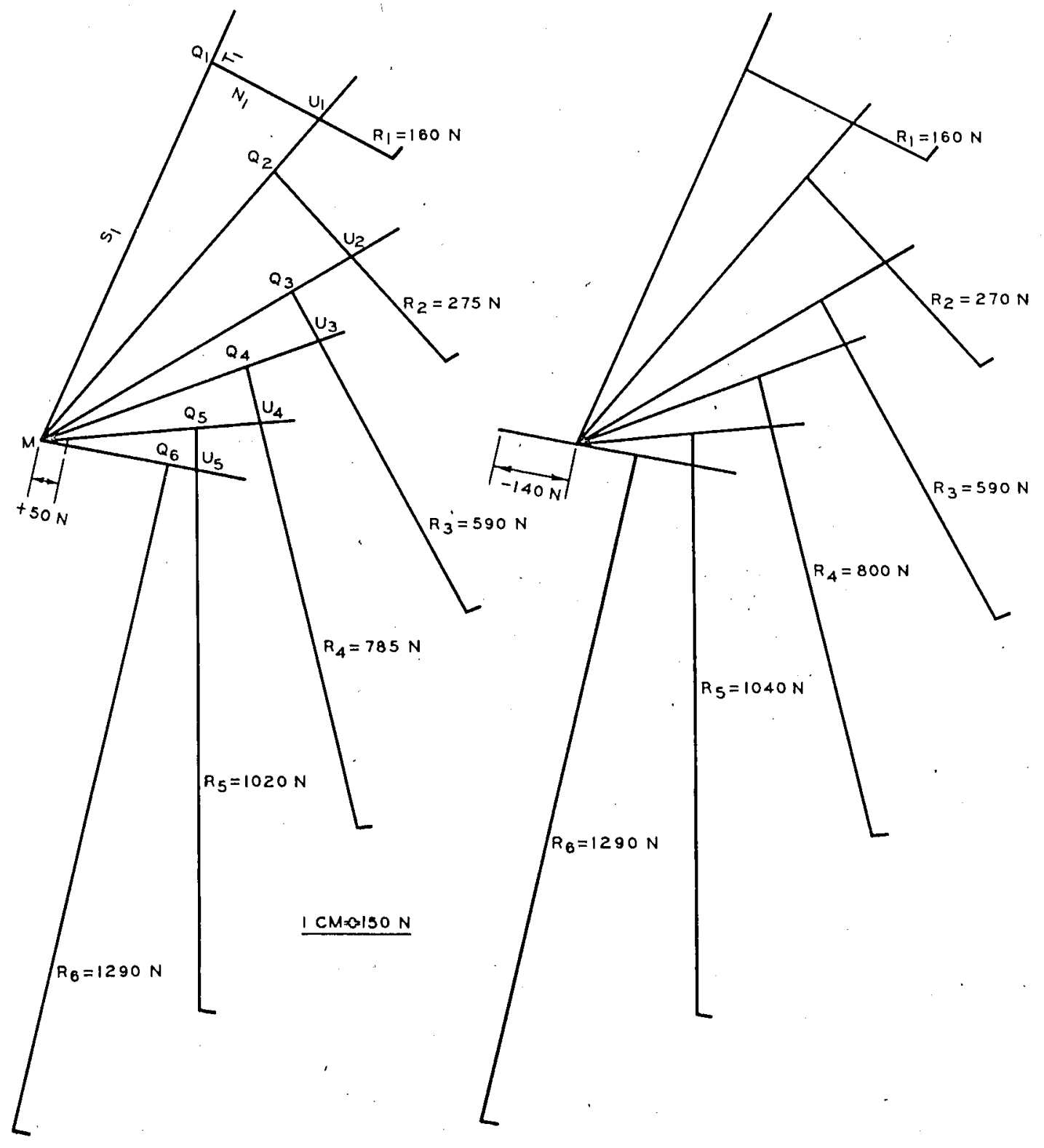

a. $k_{s}=0.15$

b. $K_{s}=0.20$

Fig. 16. Simplified solution to determine the resultant forces at the articulated joints of the experimental wheel 
54. The experimental wheel was constructed to fit into the test carriage in the U. S. Army Engineer Waterways Experiment Station mobility test facility. Programmed increasing-slip tests were performed on an air-dry sand (mortar sand), classified SP under the Unified Soil Classification System. The sand was prepared at two strength levels with penetration resistance gradients $(G)$ of approximately 2.72 and $4.53 \mathrm{~N} / \mathrm{cm}^{3}$ (0-6 in. cone index of 30 and 50, respectively). The equipment, soil preparation procedures, and test techniques have been described by McRae, Powell, and Wismer.

55. The test setup is shown in fig. 17. The television camera was arranged so that the six pressure gages and the six position indicators, i.e. the piston rods of the slave cylinders, could be photographed simultaneously on tape. Station indications were marked on the rail and also appeared on the tape so that all readings could be correlated with the stations and thus with slip. To minimize the influence of inflation pressure

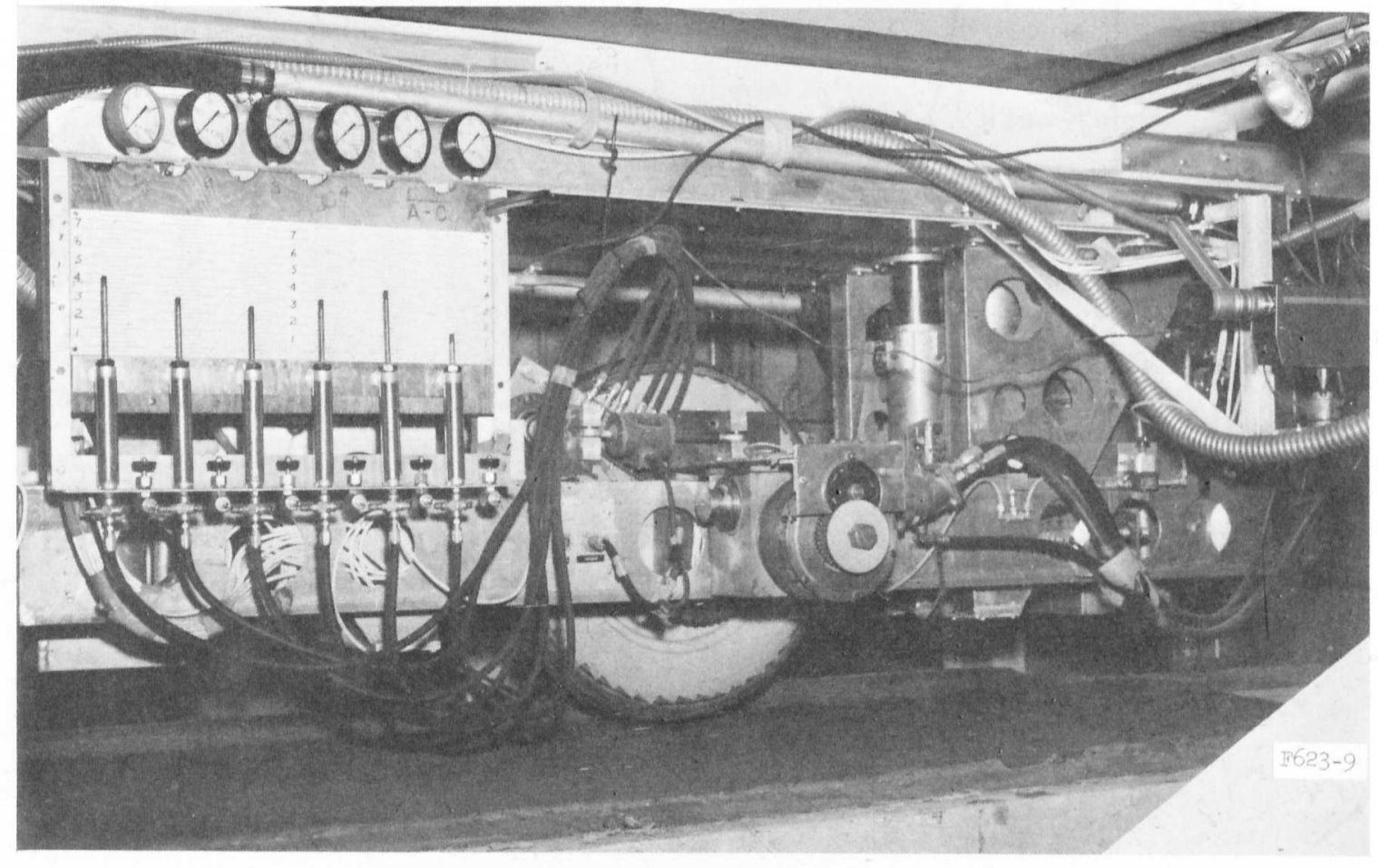

Fig. 17. Test setup 
with respect to the pressure distribution of the internal system, the tests were run with an inflation pressure of approximately $3.75 \mathrm{~N} / \mathrm{cm}^{2}$ ( 4 psi) only. With this low inflation pressure, the tire had to be held against the wheel rim by adhesive tape along its sidewalls to prevent tire-bead slippage (fig. 17). This tape was replaced after each two tests.

56. For the internal pressure distribution system to be effective, the contact length, and thus sinkage of the tire, had to be relatively large. Therefore, all tests were run with a high axle load: $4000 \mathrm{~N}$ (recommended maximum design load) for the higher strength soil and $3550 \mathrm{~N}$ for the lower strength soil. To prevent damage of the delicate internal system, the following loading procedure was adopted. Inflation pressure of approximately $12 \mathrm{~N} / \mathrm{cm}^{2}$ was applied, and the wheel was loaded with roughly half of the test load; then, the individual jacks were loaded by charging half of the test pressures at the accumulators, beginning with jack No. 6 (fig. 9). Next, the full axle load and full jack loads were applied, and, finally, the inflation pressure was lowered to $3.75 \mathrm{~N} / \mathrm{cm}^{2}$ immediately prior to the test.

57. After an initial starting distance of a few meters, during which the rotational velocity of the wheel and the carriage velocity were built up to the required level of $0.36 \mathrm{RPS}(\omega=2.26 \mathrm{radians} / \mathrm{sec})$ and $0.76 \mathrm{~m} / \mathrm{sec}$, respectively, the wheel velocity was kept constant and the carriage velocity was decreased to obtain increasing-slip conditions. With a rolling radius of $33.5 \mathrm{~cm}$, the above-mentioned velocities correspond to o percent slip. This rolling radius was determined for 25 percent tire deflection, which was an arbitrary decision. In fact, the rolling radius of the experimental wheel is very difficult to define, and so is slip, which depends on the rolling radius. In any case, true slip and true rolling radius can be determined only on the basis of the actual geometry of the soil-wheel interface, that is, only after the complete analysis of the test results. By adopting the arbitrary rolling radius of $33.5 \mathrm{~cm}$, the approximate magnitude of the slip was obtained for planning the tests. The notation of slip (as opposed to "true" siip) is used in the remainder of this report in this arbitrary sense.

58. In most tests, pressure and piston positions of the internal 
system were not completely stable at the end of the starting period, i.e. at zero slip. The later analysis of the television recordings showed that the stabilization of the internal system was usually reached at about 3-5 percent slip. The zero-slip condition could have been extended over a certain length of the test course until stabilization of the system, and the zero-slip condition could thus have been included in the analysis. But this procedure would have wasted too much of the rather restricted length $(15 \mathrm{~m})$ of the test course. Since the analysis of the test data focused on the maximum pull/load condition, which ususally occurred between 10 and 25 percent slip, and since zero slip did not describe any particular condition of the experimental wheel in any case, the extreme low slip range was not included in the analysis.

59. Toward the end of the first tests, the experimental wheel tended to break down under high slip condition, i.e. to develop a leak at the tire rim, probably because of excessive deformations. Therefore, the tests were not extended beyond approximately 50 percent slip. Thus in most cases, the actual slip tested ranged from 5 , to approximately 50 percent, which was sufficient for the purpose pursued.

60. Besides the already mentioned visual observation of piston movement and pressure variations of each cylinder of the internal system by a tape-recording camera, the measurements included oscillograph recordings of load, torque, drawbar pull, slip, vertical displacements of the wheel axle, and strain-gage readings of the sliding shoe.

61. The tests can be subdivided into four groups according to the working conditions of the internal system:

a. Tests with favorable rigidity distribution (increasing pressure from the leading to the trailing edge of the soil-wheel interface). This is the largest group.

b. Tests with unfavorable rigidity distribution (decreasing pressure along the interface).

c. Tests with neutral rigidity distribution (uniform pressure at the interface). Only a few of these tests were performed.

d. Comparison tests (conventional-tire tests) with the internal system not in operation. 


\section{Analysis Procedures}

\section{General description}

62. Three groups of data were collected and analyzed for each test:

a. Data concerning the wheel performance: load, torque, pull, and slip.

b. Data concerning the geometry of the soil-wheel interface and of the internal system: vertical axle displacement* and piston positions.

c. Data concerning the forces in the internal system: strain gage readings of the sliding shoe and accumulator pressures.

63. From the wheel performance data, the maximum pull/Ioad $(\mathrm{P} / \mathrm{W})$ ratio and the corresponding slip were determined. In this case, however, slip was only an approximation, the rolling radius being arbitrarily assumed (paragraph 57), and the determined slip value was used in the sense of a convenient marker for a particularly significant performance parameter, maximum pull/load ratio, on which the analysis focused. The efficiency, $\mathrm{E}$, was then determined at this slip value.

64. The next step was to determine the geometric conditions of the test for the slip value at maximum $\mathrm{P} / \mathrm{W}$ ratio. For this purpose, a. fullsize mechanical replica of the internal system was used (fig. 18). The recorded piston positions were reported and fixed on the replica. The geometry of the tire contour and of the internal system was then completely determined and could be, reproduced on paper. When the vertical axle

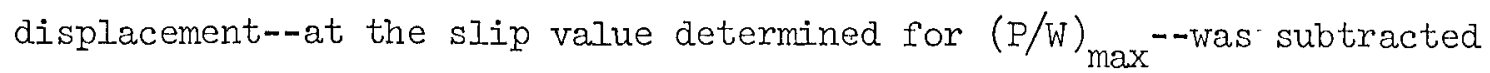
from the undeflected tire radius, the original soil surface could be drawn. Sinkage (rut depth) and contact length were graphically determined, with the bow wave being ignored (see detailed example in paragraph 71). As a

* Zero axle displacement was defined for the undeflected tire prior to each test and not for the deflected tire as usually done. Therefore, this measurement is not called "sinkage," which can only be determined later when tire deformation is considered. 


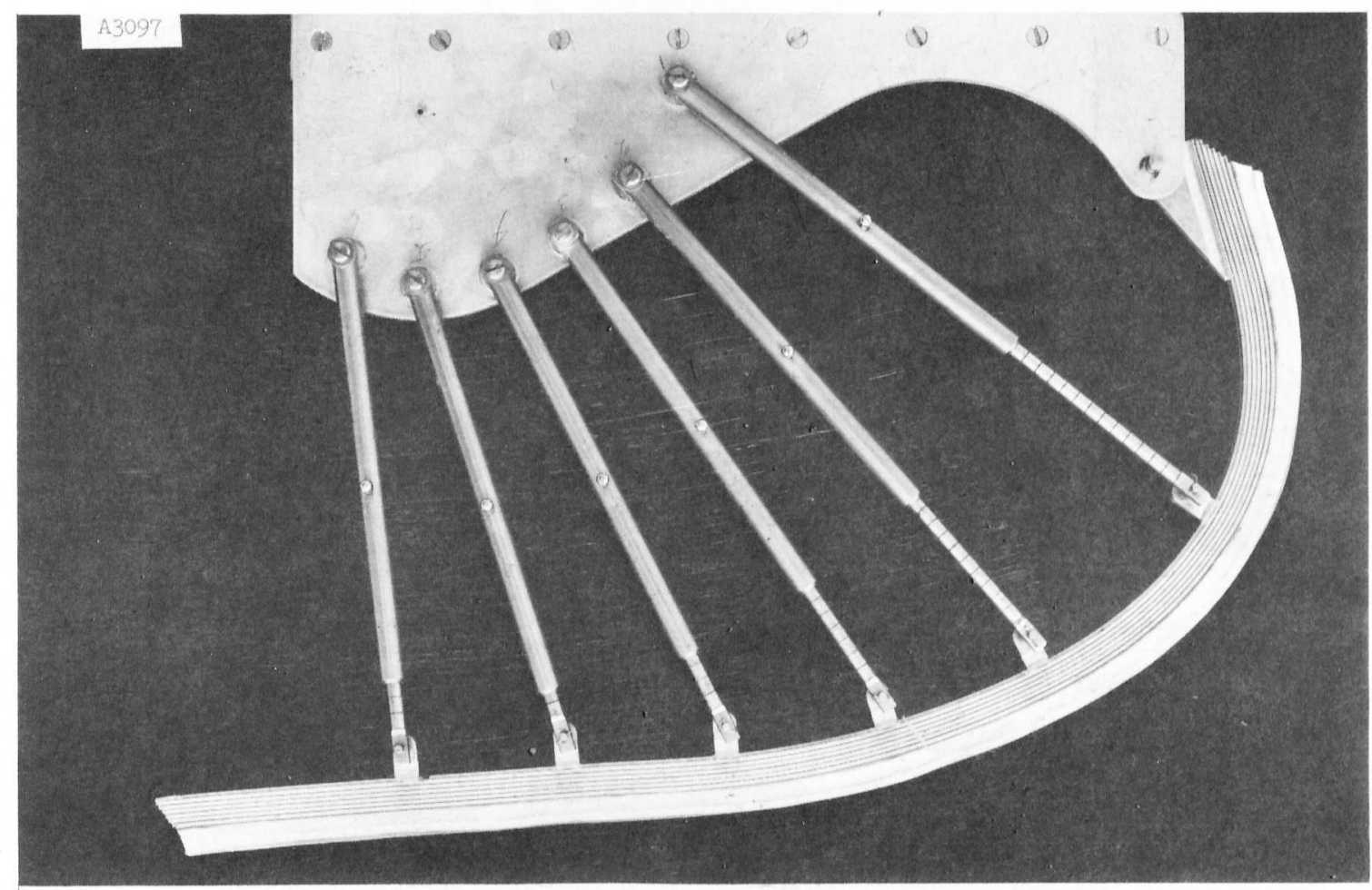

Fig. 18. Mechanical replica of the experimental wheel

simple characterization for the geometry of the soil-wheel interface, the ratio $l / z$ was determined, with $l$ being the horizontal projection of the forward part of the contact length forward of the axle and $\mathrm{z}$ being sinkage (fig. 19).

65. The geometry of the static system and the internal forces being known, the simplified graphic solution for the determination of the normal resultant forces, $R_{i}$, was applied. The resulting internal tire pressure was then computed by dividing the $R_{i}$ forces by the corresponding tire area and adding the inflation pressure, which was the same for each individual area. Finally, the internal pressure distribution over the contact length was represented in a staircase-type diagram into which the linear pressure variation line of best fit was drawn. The average pressure over the contact length was also determined from this diagram.

Detailed example

66. Raw data. To demonstrate the analysis of test data in detail and to point out some of the problems encountered during the analysis, 

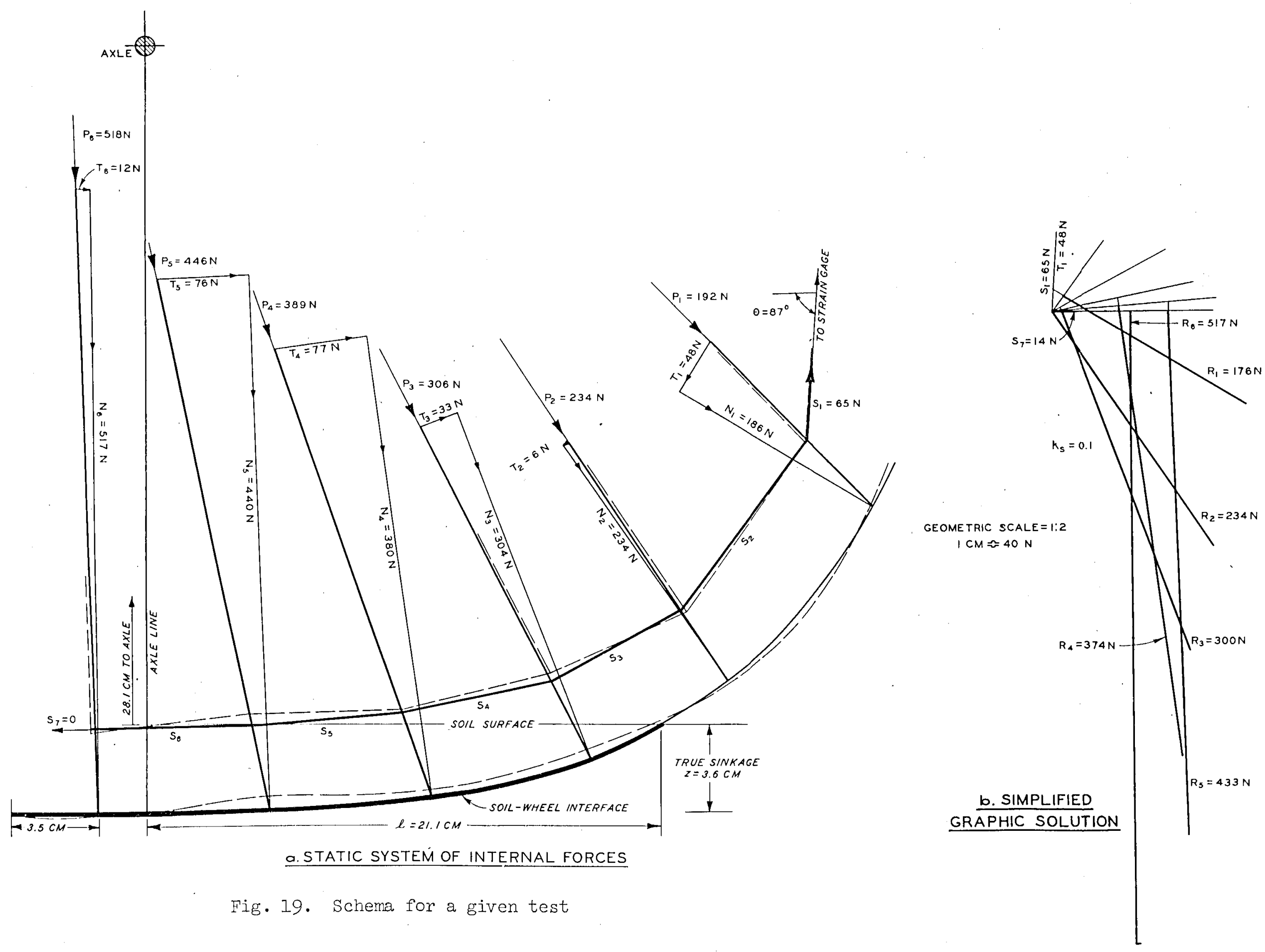
the treatment of the numerical data of one test (with favorable pressure distribution) is reproduced. The raw data for this test (No. 0011-2) are given in the following tables in the originally recorded form, i.e. using the U. S. customary units. In this test, the 0- to 6-in. cone index was 52.8, and inflation pressure was 4 psi.

67. From the wheel performance data (table 4), the pertinent performance parameters, $\mathrm{P} / \mathrm{W}$ ratio and efficiency $\mathrm{E}=\mathrm{PR}(\mathrm{I}-\mathrm{S}) / \mathrm{M}$, were computed $(\mathrm{R}=$ rolling radius $=33.5 \mathrm{~cm}) . \mathrm{P} / \mathrm{W}$ and $\mathrm{E}$ are plotted in

Table 4

Wheel Performance Data

\begin{tabular}{|c|c|c|c|c|}
\hline Station & Load (W), Ib & Pull (P), Ib & Torque (M), $\mathrm{ft} / \mathrm{Ib}$ & $\operatorname{Slip}(s), \%$ \\
\hline $0+35$ & 916 & 178 & 396 & 3.6 \\
\hline $0+40$ & 904 & 230 & 480 & 10.5 \\
\hline $0+45$ & 912 & 226 & 500 & 17.4 \\
\hline $0+50$ & 908 & 210 & 500 & 24.0 \\
\hline $0+55$ & 916 & 180 & 5.12 & 32.0 \\
\hline
\end{tabular}

fig. 20 as a function of slip. The maximum $P / W$ ratio (0.255) occurring at 11.5 percent slip, and the corresponding efficiency (0.465) were then determined from this plot (fig. 20). From table 6 (average values) the actual forces in the pistons of the master cylinders inside the wheel were computed using equation 9.

Fig. 20. Efficiency and pull// load ratio versus slip for a representative test

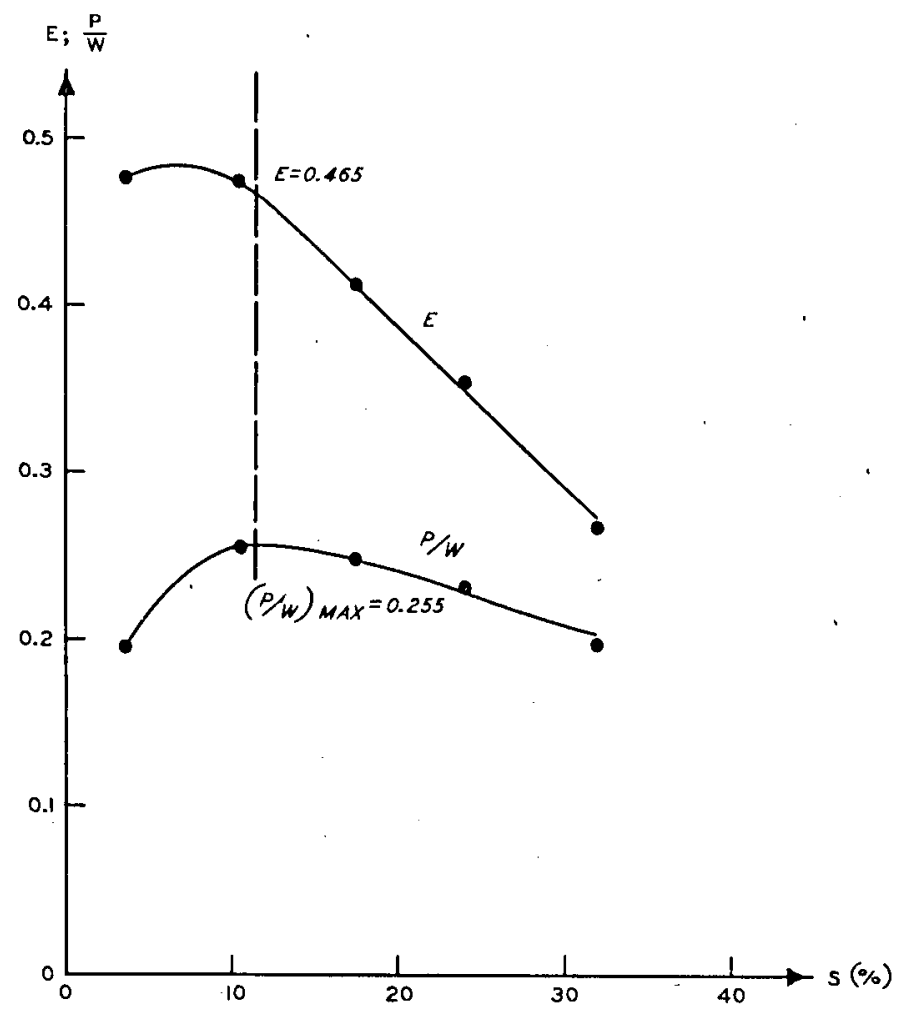


Table 5

Interface Geometry Data

\begin{tabular}{|c|c|c|c|c|c|}
\hline \multirow[b]{2}{*}{$\begin{array}{l}\text { SIip } \\
(\mathrm{s}) \\
\% \\
\end{array}$} & \multirow[b]{2}{*}{$\begin{array}{l}\text { Vertical Axle } \\
\text { Displacement } \\
\text { in. } \\
\end{array}$} & \multicolumn{4}{|c|}{ Slave Cylinder Piston Position, in. } \\
\hline & & $\begin{array}{c}\text { Cylinder } \\
\text { No. } \\
\end{array}$ & $\begin{array}{c}\text { Measured } \\
\text { by } \\
\text { Camera } \\
\end{array}$ & $\begin{array}{c}\text { Calibration } \\
\text { Position } \\
\text { (Fully Extended) } \\
\end{array}$ & $\begin{array}{l}\text { Position with } \\
\text { Respect to Full } \\
\text { Extension } \\
\end{array}$ \\
\hline 3.6 & 2.40 & 1 & 1.25 & 0.75 & 0.50 \\
\hline 10.5 & $2.56^{\circ}$ & 2 & 1.00 & 0.75 & 0.25 \\
\hline 17.4 & 2.60 & 3 & 1.50 & 0.75 & 0.75 \\
\hline 24.0 & 2.80 & $I_{t}$ & 2.00 & 0.75 & 1.25 \\
\hline 32.0 & 3.10 & 5 & 2.75 & 0.75 & 2.00 \\
\hline & & 6 & 2.25 & 0.75 & 1.50 \\
\hline
\end{tabular}

NOTE: No noticeable variation during test after starting period.

Table 6

Internal System Force Data

\begin{tabular}{|c|c|c|c|c|c|}
\hline \multicolumn{2}{|c|}{ Strain Gage } & \multicolumn{4}{|c|}{ Accumulator } \\
\hline & Tensile & \multirow[b]{2}{*}{$\begin{array}{l}\text { Accumulator } \\
\text { No. }\end{array}$} & \multicolumn{3}{|c|}{ Pressure, psi } \\
\hline $\begin{array}{l}\text { Slip } \\
(\mathrm{s}), \%\end{array}$ & $\begin{array}{l}\text { Force } \\
(\mathrm{S}), \quad 1 \mathrm{~b}\end{array}$ & & $\begin{array}{c}\text { Before } \\
\text { Test } \\
\end{array}$ & $\begin{array}{l}\text { After } \\
\text { Test } \\
\end{array}$ & Average* \\
\hline 3.6 & 20 & 1 & 59 & 59 & $58.5(40)$ \\
\hline 10.5 & 28 & 2 & 70 & 70 & $70.0(48)$ \\
\hline 17.4 & 34 & 3 & 90 & 90 & $90.0(62)$ \\
\hline 24.0 & 36 & 4 & 110 & 115 & $112.5(78)$ \\
\hline \multirow[t]{2}{*}{32.0} & 39 & 5 & 130 & 130 & $130.0(89)$ \\
\hline & & 6 & 150 & 149 & $149.5(103)$ \\
\hline
\end{tabular}

NOTE: No noticeable variation during test.

* Average pressures in. $\mathrm{N} / \mathrm{cm}^{2}$ are shown in parentheses.

Table 7

Performance Parameters

\begin{tabular}{|c|c|c|c|}
\hline Station & Pull/Load (P/W) & Efficiency (E) & Slip $(\mathrm{s}), \%$ \\
\hline $0+35$ & 0.194 & 0.476 & 3.6 \\
\hline $0+40$ & 0.254 & 0.473 & 10.5 \\
\hline $0+45$ & 0.248 & 0.411 & 17.4 \\
\hline $0+50$ & 0.231 & 0.352 & 24.0 \\
\hline $0+55$ & 0.196 & 0.264 & 32.0 \\
\hline
\end{tabular}


68. Determination of the geometric conditions. The geometry of the soil-wheel interface was determined by means of the full-scale mechanical. model of the internal system (fig. 18). The positions of the pistons were fixed from the results of table 5 (last column); the interface contour and the piston orientation were thus completely defined and were drawn on paper. The dashed lines in fig. 19 show the result of this operation. The interface contour is slightly undulating, a result that is thought to be unreasonable because the internal pressure distribution is monotonously increasing over the contact length. There is no reason either for the external soil reaction forces to present local maxima (assuming once more that the local rigidity is fully under control; that is, without interference of carcass stiffness or tire flexing). The apparent undulation of the interface, as determined from the piston position readings, undoubtedly is caused by leakage of the internal system and therefore does not reflect the actual conditions.

69. In fact, the correlation between the slave cylinder pistons and the interface geometry is based upon the premise of a fixed amount of incompressible hydraulic fluid in the internal system (fig. 12). When the system was recalibrated after a certain number of tests, the amount of fluid in some of the cylinders had changed slightly and unsystematically. The lubrication fluid in the tire probably entered into some cylinders; others lost part of the hydraulic fluid. Air bubbles are also likely to have developed during the tests; therefore, the determination of the tire geometry was not overly reliable. The reliability of the system could have been increased without major modifications only by recalibrating the system after each test. This, however, would have been too time-consuming, since the wheel had to be completely dismantled for the calibration. Also, this deficiency had been discovered only after half of the test program was completed. Later in the program, a method was developed that allowed a more frequent calibration without actual dismantling of the assembly.

70. In cases in which the determined tire geometry was apparentIy in error, the following procedure was followed. The fixing screws (fig. 18) of the model's pistons visually creating the most buckling were released. In the case of fig. 19a, these were pistons 3 and 5 . The elasticity of 
the shoe then made the model assume another configuration, presenting a much smoother contour of the interface. These screws were then retightened. In the case of fig. 19a, the procedure was repeated with pistons 2 and 4, resulting in the solid-line configuration of fig. 19a, which was used for the further analysis. This procedure implies that the overall features of the interface were adequately represented by the original setting. Fig. 19a shows that the geometric differences between the original and the smoothed-out configuration are minor. The piston forces themselves are measured independently and are thus not affected by this correlation, so the effect on the final result of the described operation remains negligible.

71. The recorded vertical displacement (table 5) for 11.5 percent slip was $2.57 \mathrm{in.}(\approx 6.5 \mathrm{~cm})$. The actual soil surface was reconstructed by subtracting the axle displacement from the undeflected tire radius: 34.6 $6.5=28.1 \mathrm{~cm}$ ( $\mathrm{fig}$. 19a). The true sinkage $(\mathrm{z})$ was then determined from fig. 19a to be $3.6 \mathrm{~cm}$, and the horizontal projection of the forward part of the contact length $(\ell)$ to be $21.1 \mathrm{~cm}$ (ignoring the bow wave). To characterize roughly the geometry of the interface, the ratio $\ell / z=21.1 / 3.6=$ 5.85 was used.

72. By connecting the end points of the pistons (articulations in fig. 9), the line of action of the $s_{i}$ forces was determined (fig. 19a). Finally, the piston lines were prolonged until they intersected with the interface. At the point of intersection, the normals to the interface were drawn. The geometric conditions were now known. In the given case, only pistons 3-6 actually fell within the contact length and contributed to the local rigidity. In all tests, piston 1 was outside the interface. The tire surface area corresponding to piston 6 was assumed fully in contact. with the soil, so that the contact length extended backward beyond the last piston for $3.5 \mathrm{~cm}$ (see fig. 19a).

73. At this point of the analysis, the question arose as to how the rolling radius and thus the slip should be defined. In the case in fig. 19a, the smallest distance from the axle to the interface happens to coincide with the vertical radius beneath the axle, equal to $31.7 \mathrm{~cm}$. By taking this value for the rolling radius $\left(R_{a}\right)$, the computed slip would be: 


$$
s_{a}=1-(I-s) \frac{R}{R_{a}}=1-(1-0.115) \frac{33.5}{31.7}=0.065,
$$

as compared with $s=0.115$ previously obtained.

74. It has always been recognized, however, that any definition of the rolling radius for deforming tires on yielding soils is arbitrary. Besides the smallest and the vertical radius (which did not always coincide), the mean radius over the contact length or the radius for the half contact angle could be proposed and justified equally well. Since actual rolling radius and slip were not essential for the evaluation of the test results, these data were not included in the analysis. In this context, it is important to note that the efficiency as defined in paragraph 28 does not depend on the definition of slip and rolling radius, as seen by the first expression of $E$ in equation 5. All the elements of the first expression are directly measurable in the test facility. The second expression can be derived from these measurements if the rolling radius, which, in turn, determines the slip value, is known.

75. Determination of local rigidity. The piston forces (table 8) Table 8

Piston Forces

\begin{tabular}{|c|c|c|c|}
\hline $\begin{array}{c}\text { Piston } \\
\text { Noo. } \\
\end{array}$ & $\begin{array}{l}\text { Force } \\
\text { (N) }\end{array}$ & $\begin{array}{l}\text { Piston } \\
\text { No. } \\
\end{array}$ & $\begin{array}{l}\text { Force } \\
(\mathrm{N})\end{array}$ \\
\hline 1 & 192 & 4 & 389 \\
\hline 2 & 234 & 5 & 446 \\
\hline 3 & 306 & 6 & 518 \\
\hline
\end{tabular}

were separated into their normal and tangential components (fig. 19a). Correctly, vectors $\mathrm{N}_{i}$ and $\mathrm{T}_{i}$ should have been drawn so as to intersect with vectors $\mathrm{S}_{i}$. However, for the sake of clarity, the presentation in fig. 19a was preferred. The strain gage reading at slip $s=0.115$ was interpolated from table 6 to be $S=291 \mathrm{~b}=129 \mathrm{~N}$. Force $S_{1}$ was determined from equation 11 as $S_{I}=65 \mathrm{~N}$, with the angle $\theta$ being graphically determined from fig. 19a.

76. The simplified graphic solution developed in paragraphs $49-53$ and described in detail in Appendix $A$ is shown in fig. 19b. The heavy 
Iines represent the resulting forces $R_{1}-R_{6}$, which are not shown in fig. 19a. (See figs. 13 and 14 for the complete force system.) The assumed friction coefficient of $k_{S}=0.1$ turned out to be a good estimate, since the remaining force $S_{7}=14 \mathrm{~N}$ was negligibly small in comparison with the acting forces. Further refinement of the solution would not have changed the magnitude of the $R_{i}$ forces appreciably. For the example presented, the difference between corresponding $N_{i}$ and $R_{i}$ forces is minor. This, however, is not the general case.

77. The corresponding tire'surface for every piston (except for pistons 1 and 2) was approximately $7 \mathrm{~cm}$ by $11 \mathrm{~cm}=77 \mathrm{~cm}^{2}$, within the smaller dimension in the direction of travel. The total local tire pressure (equivalent to rigidity) was obtained by dividing the $R_{i}$ forces by the corresponding tire area and adding the inflation pressure. The results of these computations are tabulated below and shown in a staircase diagram in fig. 21 .

Table 9

Determination of Local Rigidity

\begin{tabular}{|c|c|c|c|c|c|}
\hline $\begin{array}{c}\text { Piston } \\
\text { No. } \\
\end{array}$ & $\begin{array}{c}R_{i} \text { Forces } \\
\mathrm{N} \\
\end{array}$ & $\begin{array}{c}\text { Tire Area } \\
\mathrm{cm}^{2} \\
\end{array}$ & $\begin{array}{c}\text { Pressure } \\
\text { Caused by } R_{i} \\
N / \mathrm{cm}^{2} \\
\end{array}$ & $\begin{array}{c}\text { Inflation } \\
\text { Pressure } \\
\mathrm{N} / \mathrm{cm}^{2} \\
\end{array}$ & $\begin{array}{l}\text { Total } \\
\text { Pressure } \\
\mathrm{N} / \mathrm{cm}^{2} \\
\end{array}$ \\
\hline 1 & 176 & 110 & 1.60 & 2.76 & 4.36 \\
\hline 2 & 234 & 93.5 & 2.50 & 2.76 & 5.26 \\
\hline 3 & 300 & 77 & 3.89 & 2.76 & 6.65 \\
\hline 4 & 374 & 77 & 4.86 & 2.76 & 7.62 \\
\hline 5 & 433 & 77 & 5.62 & 2.76 & 8.38 \\
\hline 6 & 517 & 77 & 6.71 & 2.76 & 9.47 \\
\hline
\end{tabular}

78. The average internal pressure over the contact length was determined to be $\sigma_{a}=8.03 \mathrm{~N} / \mathrm{cm}^{2}$, and the pressure variation within the contact length (rigidity variation) to be $0.13 \mathrm{~N} / \mathrm{cm}^{2} / \mathrm{cm}$. In cases in which the contact length did not coincide with a full individual piston area 


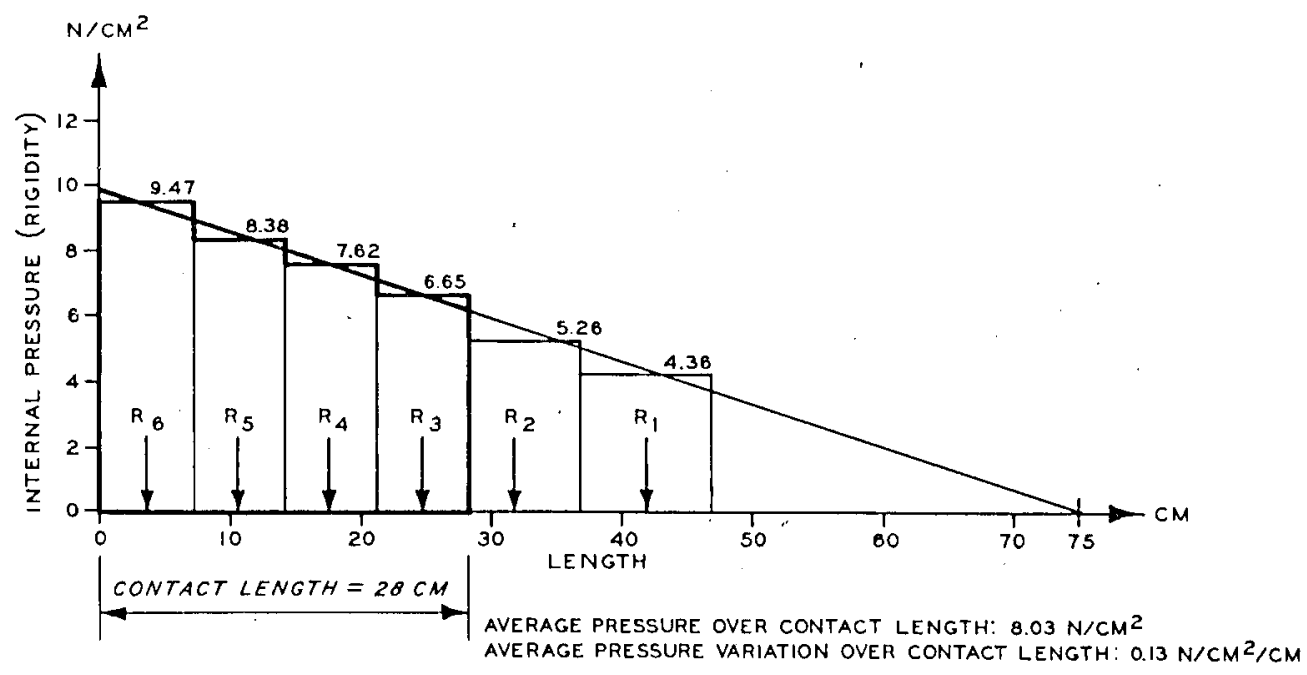

Fig. 21. Internal tire pressure (rigidity) distribution

length, the proportion of the area within the contact length was taken into consideration.

\section{Final Test Results}

\section{Presentation}

79. Approximately 70 tests were conducted with the internal system in operation. In addition, about 20 base-line tests, in which the tire was used as a conventional tire with various inflation pressures, were run for comparison purposes. In these comparison tests, the internal hydraulic. system was not charged; the inflation pressure pushed the master pistons inside the wheel upward ( $f i g .12$ ) so that the sliding shoe actually was not in contact with the tire. Therefore, no geometric data on the soil-wheel interface could be recorded for the base-line tests. Because of the complexity of the experimental wheel and the delicate test technique, 32 tests had to be disregarded for various reasons. A total of 58 tests (26 with favorable pressure distribution, 13 with unfavorable distribution, 4 with neutral pressure distribution, and 15 base-line tests) could be considered successful and were analyzed as described in the preceding paragraphs. The final results are listed in tables 10 and 11 .

80. The $(\mathrm{P} / \mathrm{W})_{\max }$ ratio is plotted for the medium-dense and dense 
Table 10

Final Test Results on Medium-Dense Sand

Penetration Resistance Gradient $\approx 2.72 \mathrm{~N} / \mathrm{cm}^{3}$

$0-6$ in. $C I \approx 30$; Axle Load $W=3550 \mathrm{~N}$

\begin{tabular}{|c|c|c|c|c|c|c|c|c|}
\hline $\begin{array}{c}\text { Test } \\
\text { No. } \\
\end{array}$ & $\begin{array}{c}\text { Penetration } \\
\text { Resistant } \\
\text { Gradient } \\
\quad \mathrm{N} / \mathrm{cm}^{3} \\
\end{array}$ & $\begin{array}{c}\text { Maximum } \\
\text { PulI/Load } \\
(\mathrm{P} / \mathrm{W}) \\
\end{array}$ & $\begin{array}{l}\text { Effi- } \\
\text { ciency } \\
(\mathrm{E}) \\
\end{array}$ & $\begin{array}{c}\text { Sink- } \\
\text { age } \\
(\mathrm{z}) \\
\mathrm{cm} \\
\end{array}$ & $\begin{array}{l}\text { Forward } \\
\text { Length/ } \\
\text { Sinkage } \\
(\ell / z) \\
\end{array}$ & $\begin{array}{c}\text { Slip } \\
(\mathrm{s}) \\
\% \\
\end{array}$ & $\begin{array}{c}\text { Average } \\
\text { Pres- } \\
\text { sure } \\
\mathrm{N} / \mathrm{cm}^{2} \\
\end{array}$ & $\begin{array}{l}\text { Pressure } \\
\text { Increase } \\
\mathrm{N} / \mathrm{cm}^{2} / \mathrm{cm}\end{array}$ \\
\hline $0057 \mathrm{~B}$ & 2.76 & 0.16 & 0.255 & 6.3 & 3.4 & 20 & 8.7 & 0.14 \\
\hline $0058 \mathrm{~B}$ & 2.81 & 0.13 & 0.22 & 3.2 & 6.0 & 20 & 9.6 & 0.175 \\
\hline $0059 B$ & 2.67 & 0.175 & 0.28 & 6.7 & 2.8 & 20 & 8.25 & 0.09 \\
\hline $0060 \mathrm{~B}$ & 2.68 & 0.215 & 0.325 & $5 \cdot 3$ & 3.95 & 21.5 & 7.20 & 0.08 \\
\hline $0062 B$ & 2.94 & 0.245 & 0.40 & 4.4 & 4.7 & 22 & 6.30 & 0.11 \\
\hline $0064 \mathrm{~B}$ & 2.66 & 0.245 & 0.355 & 4.2 & 4.7 & 20 & 6.1 & 0 \\
\hline $0066 \mathrm{~B}$ & 2.84 & 0.24 & 0.38 & 4.1 & 5.1 & 22 & 6.55 & 0.165 \\
\hline $0067 \mathrm{~B}$ & 2.72 & 0.225 & 0.36. & 5.4 & 4.4 & 21.5 & 6.45 & 0.14 \\
\hline $0069 B$ & 2.66 & 0.24 & 0.375 & 4.4 & 4.85 & 20 & 6.30 & 0.13 \\
\hline $0070 \mathrm{~B}$ & 2.72 & 0.19 & 0.32 & 5.3 & 4.65 & 16.5 & 7.0 & -0.09 \\
\hline $0071 B$ & 2.82 & 0.25 & 0.39 & 4.4 & 4.7 & 22 & 6.25 & 0.11 \\
\hline $0072 B$ & 2.79 & 0.225 & 0.345 & $4 \cdot 7$ & 4.0 & 24 & 6.5 & 0.20 \\
\hline $0073 B$ & 2.86 & 0.235 & 0.37 & 4.8 & 4.7 & 18 & 6.15 & 0 \\
\hline $0074 \mathrm{~B}$ & 2.73 & 0.24 & 0.38 & $4 \cdot 3$ & 5.1 & 20 & 6.2 & 0.13 \\
\hline $0075 B$ & 2.80 & 0.185 & 0.31 & 4.7 & 5.2 & $17 \cdot 5$ & 7.15 & -0.115 \\
\hline $0076 \mathrm{~B}$ & 2.76 & 0.245 & 0.38 & 4.4 & 5.0 & 22 & 6.0 & 0.13 \\
\hline $0077 \mathrm{~B}$ & 2.65 & 0.175 & 0.285 & 5.9 & 4.1 & 17 & 7.4 & -0.10 \\
\hline $0078 \mathrm{~B}$ & 2.80 & 0.155 & 0.26 & 5.6 & 3.4 & 20 & 8.75 & 0.135 \\
\hline OO79B & 2.67 & 0.17 & 0.275 & 5.5 & 4.1 & 17 & 7.8 & -0.12 \\
\hline $0001-2$ & 3.00 & 0.245 & 0.435 & 4.6 & $4 \cdot 3$ & 15 & 5.6 & 0.10 \\
\hline $0004-2$ & 2.81 & 0.245 & 0.385 & 6 & 3.9 & 17 & 5 & -0.065 \\
\hline $0052 B$ & 2.78 & 0.12 & 0.225 & \multirow{6}{*}{\multicolumn{2}{|c|}{$\begin{array}{l}\text { Comparison } \\
\text { tests 'with } \\
\text { no pres- } \\
\text { sure in } \\
\text { the inter- } \\
\text { nal system }\end{array}$}} & 20 & 10.35 & -- \\
\hline $0053 \mathrm{~B}$ & 2.67 & 0.175 & 0.32 & & & 20 & 8.20 & - \\
\hline $0054 \mathrm{~B}$ & 2.73 & 0.215 & 0.385 & & & 20 & 6.90 & -- \\
\hline OOBOB & 2.74 & 0.25 & 0.41 & & & 20 & 5.5 & - \\
\hline $0081 \mathrm{~B}$ & 2.72 & 0.285 & 0.455 & & & 22 & 4.15 & - \\
\hline $0082 B$ & 2.84 & 0.305 & 0.455 & & & 22 & 3.45 & -- \\
\hline
\end{tabular}
NOTE: Inflation pressure for tests $0001-2$ and $0004-2$ was $2.07 \mathrm{~N} / \mathrm{cm}^{2}$, instead
of $2.76 \mathrm{~N} / \mathrm{cm}^{2}$. 
Table 11

Final Test Results on Dense Sand.

Penetration Resistance Gradient $G \approx 4.54 \mathrm{~N} / \mathrm{cm}^{3}$

$0-6$ in. $C I=50 ;$ Axle Load $\mathrm{W}=4000 \mathrm{~N}$

\begin{tabular}{|c|c|c|c|c|c|c|c|c|}
\hline $\begin{array}{r}\text { Test } \\
\text { No. }\end{array}$ & $\begin{array}{c}\text { Penetration } \\
\text { Resistant } \\
\text { Gradient } \\
\quad \mathrm{V} / \mathrm{cm}^{3} \\
\end{array}$ & $\begin{array}{l}\text { Maximum } \\
\text { PuII/Load } \\
(\mathrm{P} / \mathrm{W}) \\
\end{array}$ & $\begin{array}{l}\text { Effi- } \\
\text { ciency } \\
(\mathrm{E}) \\
\end{array}$ & $\begin{array}{l}\text { Sinli- } \\
\text { age } \\
(\mathrm{z}) \\
\mathrm{cm} \\
\end{array}$ & $\begin{array}{l}\text { Forward } \\
\text { Length/ } \\
\text { Sinkage } \\
(\ell / z) \\
\end{array}$ & $\begin{array}{c}\text { Slip } \\
(\mathrm{s}) \\
\% \\
\end{array}$ & $\begin{array}{c}\text { Average } \\
\text { Pres- } \\
\text { sure } \\
\mathrm{N} / \mathrm{cm}^{2} \\
\end{array}$ & $\begin{array}{l}\text { Pressure } \\
\text { Increase } \\
\mathbb{N} / \mathrm{cm}^{2} / \mathrm{cm} \\
\end{array}$ \\
\hline $0089 \mathrm{~B}$ & 4.88 & 0.15 & 0.30 & 5.6 & 3.4 & 10 & 22.8 & 0.23 \\
\hline OO9OB & 5.03 & 0.16 & 0.31 & 3.6 & 5.3 & 12 & 12.9 & 0.235 \\
\hline $0091 B$ & 4.81 & 0.165 & 0.315 & 4.0 & 4.7 & 11.5 & 12.0 & 0.30 \\
\hline $0092 B$ & 4.63 & 0.16 & 0.29 & $4 \cdot 3$ & 4.2 & 14 & 12.0 & 0.275 \\
\hline O093B & 4.75 & 0.18 & 0.35 & 4.8 & 3.8 & 12 & 11.7 & 0.20 \\
\hline $0.09 l_{4} B$ & 4.74 & 0.175 & 0.335 & 4.0 & 4.7 & 11. & 11.7 & 0.175 \\
\hline $0095 B$ & 4.97 & 0.185 & 0.325 & 5.5 & 3.5 & 12 & 11.1 & 0.06 \\
\hline $0096 \mathrm{~B}$ & 4.74 & 0.175 & 0.32 & 4.1 & 4.9 & 12 & 11.25 & 0.065 \\
\hline $0097 \mathrm{~B}$ & 4.42 & 0.175 & 0.33 & 4.1 & 4.8 & 10.5 & 10.3 & -0.06 \\
\hline $0098 \mathrm{~B}$ & 4.25 & 0.195 & 0.35 & 5 & 4.1 & 12 & 10.2 & 0 \\
\hline $0099 B$ & 4.83 & 0.195 & 0.325 & 3 & 6.7 & 1.0 & 9.15 & -0.125 \\
\hline OIOIB & 4.99 & 0.185 & 0.315 & 3.4 & 6.35 & 14 & 9.75 & -0.2 \\
\hline $0010-2$ & 4.74 & 0.285 & 0.475 & 4.2 & 5.5 & 1.1 .5 & 6.9 & 0.09 \\
\hline OOLI-2 & 4.79 & 0.255 & 0.465 & 3.6 & 5.85 & 11.5 & 8.05 & 0.13 \\
\hline $0012-2$ & 4.76 & 0.275 & 0.485 & 4.0 & 6.75 & 13.0 & 7.5 & 0.07 \\
\hline $0013-2$ & 4.76 & 0.26 & 0.405 & 3.8 & 6.15 & 14 & 7.75 & -0.1 \\
\hline $0015-2$ & 4.42 & 0.205 & 0.37 & 4.8 & 4.7 & 12.5 & 9.4 & -0.09 \\
\hline $0016-2$ & 4.69 & 0.22 & 0.38 & 5.1 & $4 \cdot 3$ & 14 & 9.6 & 0.07 \\
\hline $0017-2$ & 4.78 & 0.245 & 0.425 & 4.9 & 4.6 & 13 & 8.0 & -0.115 \\
\hline $0018-2$ & 4.74 & 0.27 & 0.465 & 3.6 & 6.1 & 12 & 7.0 & 0 \\
\hline $0019-2$ & 4.73 & 0.26 & 0.415 & 4.5 & 4.95 & 14 & 7.25 & -0.1 \\
\hline $0021-2$ & 4.64 & 0.13 & 0.25 & 4.9 & $4 \cdot 3$ & 13 & 11.7 & -0.14 \\
\hline $0103 B$ & 4.87 & 0.24 & 0.46 & \multirow{9}{*}{\multicolumn{2}{|c|}{$\begin{array}{l}\text { Comparison } \\
\text { tests with } \\
\text { no pressure } \\
\text { in the in- } \\
\text { ternal } \\
\text { system }\end{array}$}} & 15 & 8.3 & -- \\
\hline $0104 \mathrm{~B}$ & 4.86 & 0.265 & 0.51 & & & 13.5 & 8.3 & - \\
\hline $0105 B$ & 4.92 & 0.285 & 0.515 & & & 15 & 6.2 & - \\
\hline $0106 \mathrm{~B}$ & 5.05 & 0.295 & 0.53 & & & 13 & 6.2 & - \\
\hline O108B & 4.91 & 0.30 & 0.53 & & & 16 & 4.8 & -- \\
\hline $0005-2$ & 4.60 & 0.145 & 0.35 & & & 16 & 13.75 & -- \\
\hline $0006-2$ & 4.40 & 0.16 & 0.35 & & & 16 & 12.4 & -- \\
\hline $0007-2$ & 4.48 & 0.30 & 0.485 & & & 17.5 & 4.8 & -- \\
\hline $0009-2$ & 4.86 & 0.295 & 0.49 & & & 16.0 & 4.8 & -- \\
\hline
\end{tabular}

NOTE: Tests $O 089 B$ through $0101 B$ were programmed with assumed rolling radius $R=23.5$, instead of $R=33.5$. 
sands as a function of the average pressure $\sigma_{a}$ in figs. 22 and 23, respectively, with the magnitude of the pressure (rigidity) variation as a parameter represented by the length and direction of the arrows. The main factor governing the $(\mathrm{P} / \mathrm{W})_{\max }$, value is the average pressure $\sigma_{a}$ : the performance increases with decreasing average pressure, which is a known result. However, within this general trend, the results for tests with favorable and unfavorable pressure distribution (upward and downward arrows, respectively) clearly separate into two distinct curves, as shown by the dashed lines. For both soils, the favorable pressure distribution yields significantly higher $(\mathrm{P} / \mathrm{W})_{\max }$ values than the unfavorable pressure distribution for the same average pressure. To clearly outperform the conventionally operating tire (open circles in figs. 22' and 23) was impossible, however, although a slight tendency in that direction might be observed in the range of low average pressures.* The rate of internal pressure variation, as indicated by the length of the arrows in these figures, is unrelated to the performance of the tires. The data on tests with neutral pressure distributions are too scarce to allow a firm statement.

81. The efficiency at. $(\mathrm{P} / \mathrm{W})_{\max }$ is plotted in an analogous manner in figs. 24 and 25 for the medium-dense and dense sands, respectively. In these plots, the scatter of the data is larger than for the $(\mathrm{P} / \mathrm{W})_{\max }$ values. This, however, is normal since both the energy input and output data are reflected in the efficiency. The highly complex internal system of the experimental wheel absorbs part of the input energy in a more-or-. less uncontrolled manner. For this reason, the conventional tire clearly operates with a higher efficiency, except in the range of low average pres- . sures. Nevertheless, if the scatter region is delineated and hatched separately for favorable and unfavorable pressure distributions, both regions are clearly distinguishable without overlapping. The favorable pressure distribution persistently yields a higher efficiency than the unfavorable

* Because of tire flexing and sidewall stiffness, the mean rigidity for these tests is higher than the inflation pressure, especially in the range of low average pressures. Consideration of this fact would shift the conventional-tire data (open circles in figs. 22-25) in the low average pressure range to the right, and thus produce a more consistent picture. 


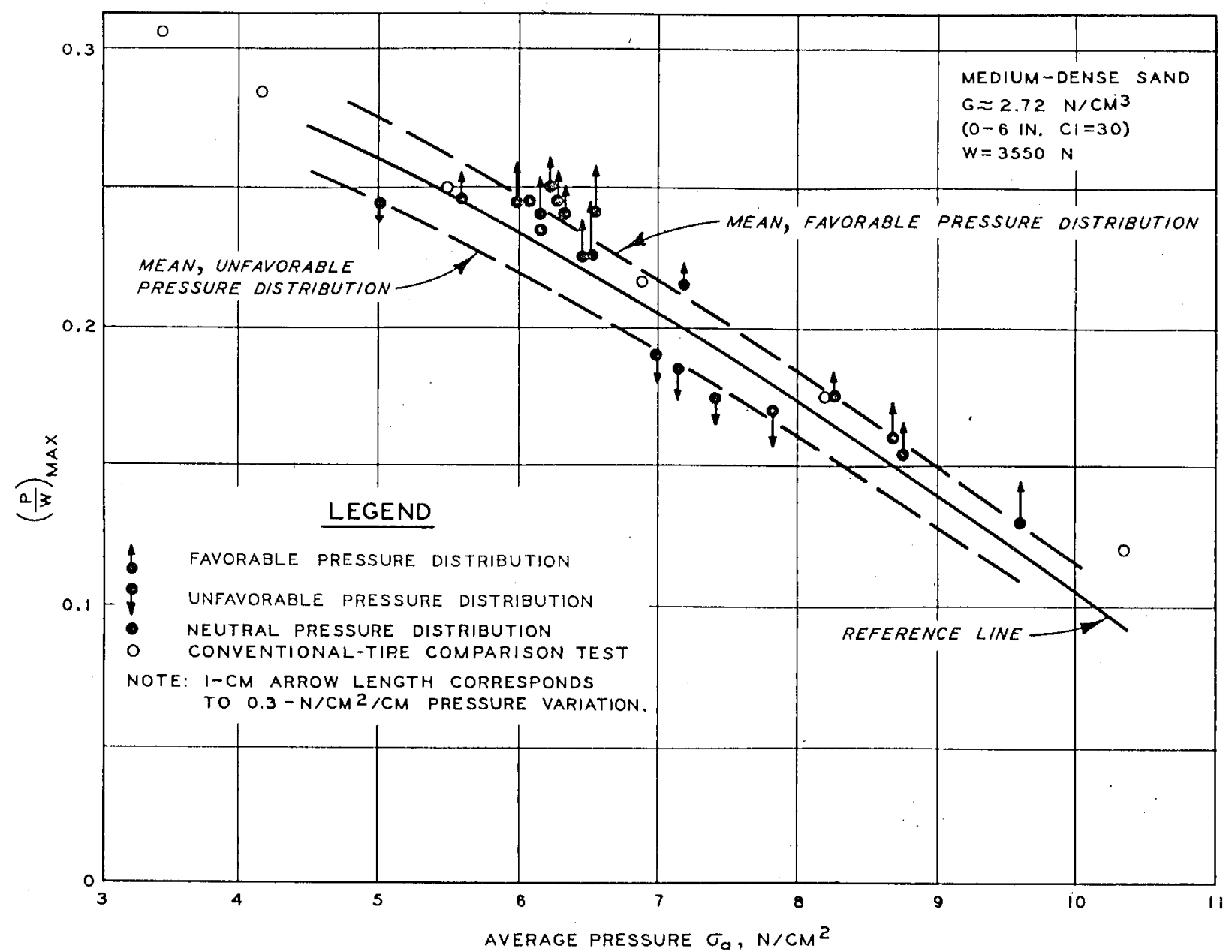

Fig. 22. Variation of maximum pull/load ratio; medium-dense sand 


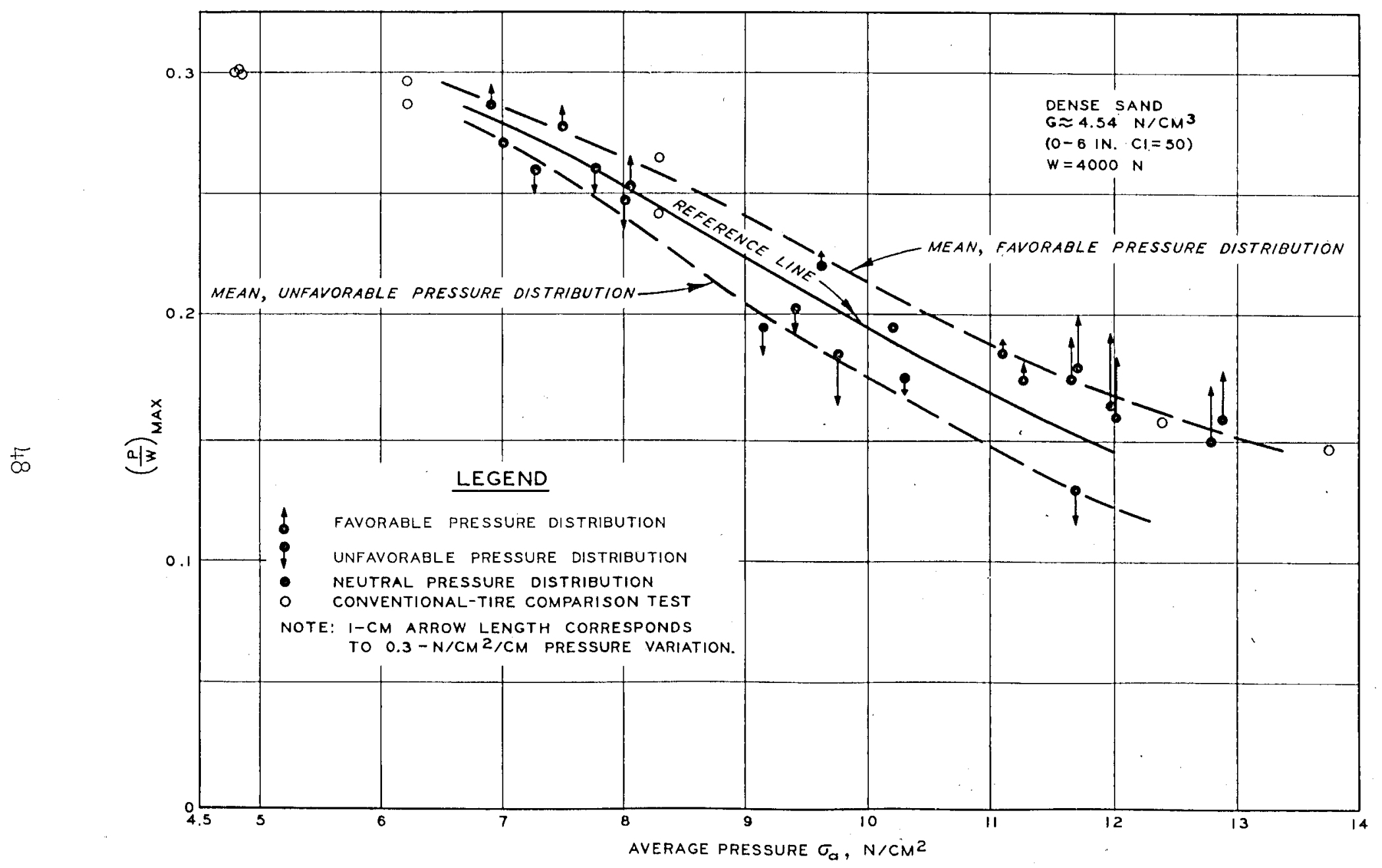

Fig. 23. Variation of maximum pull/load ratio; dense sand 


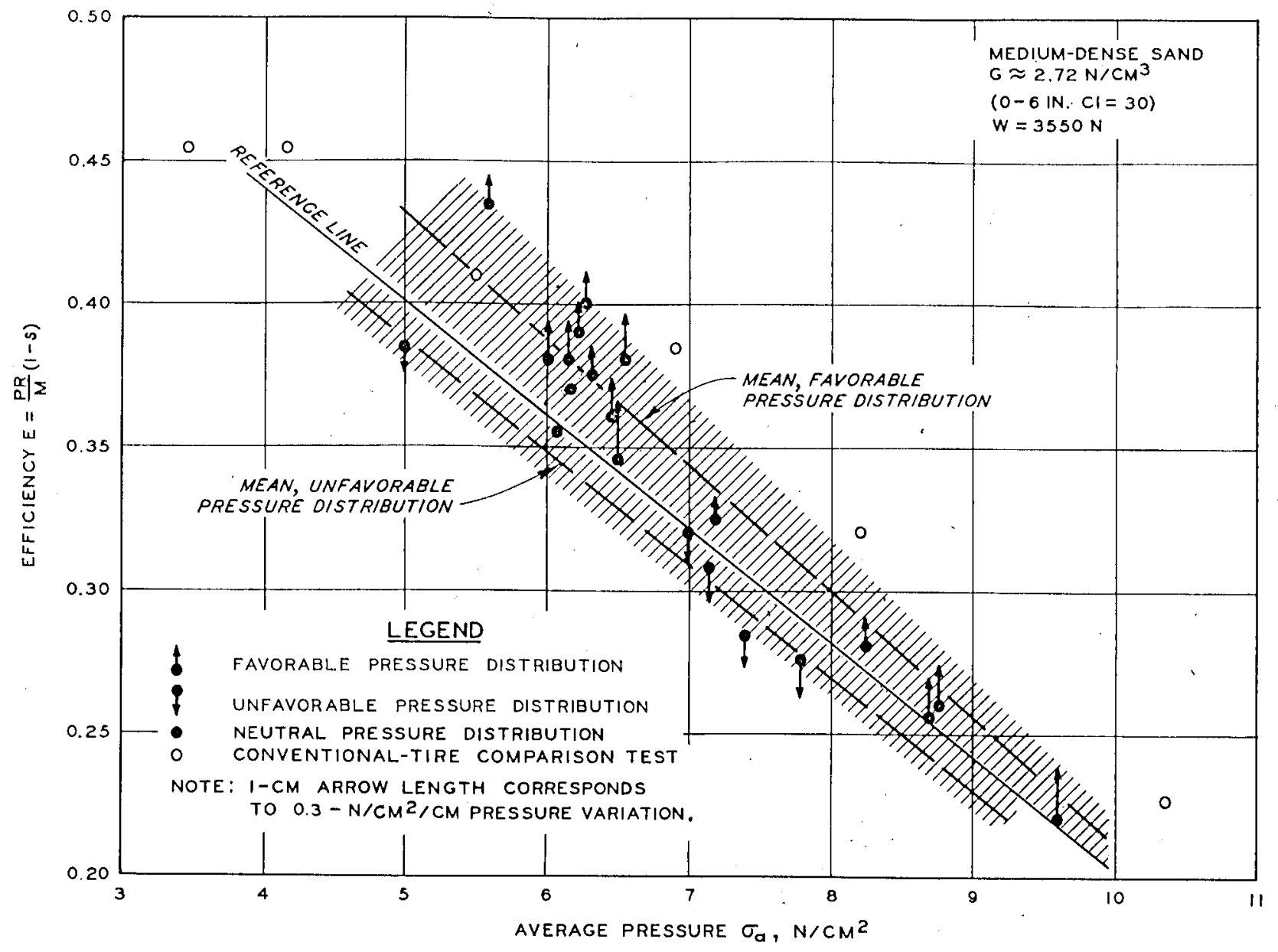

Fig. 24: Variation of efficiency; medium-dense sand 


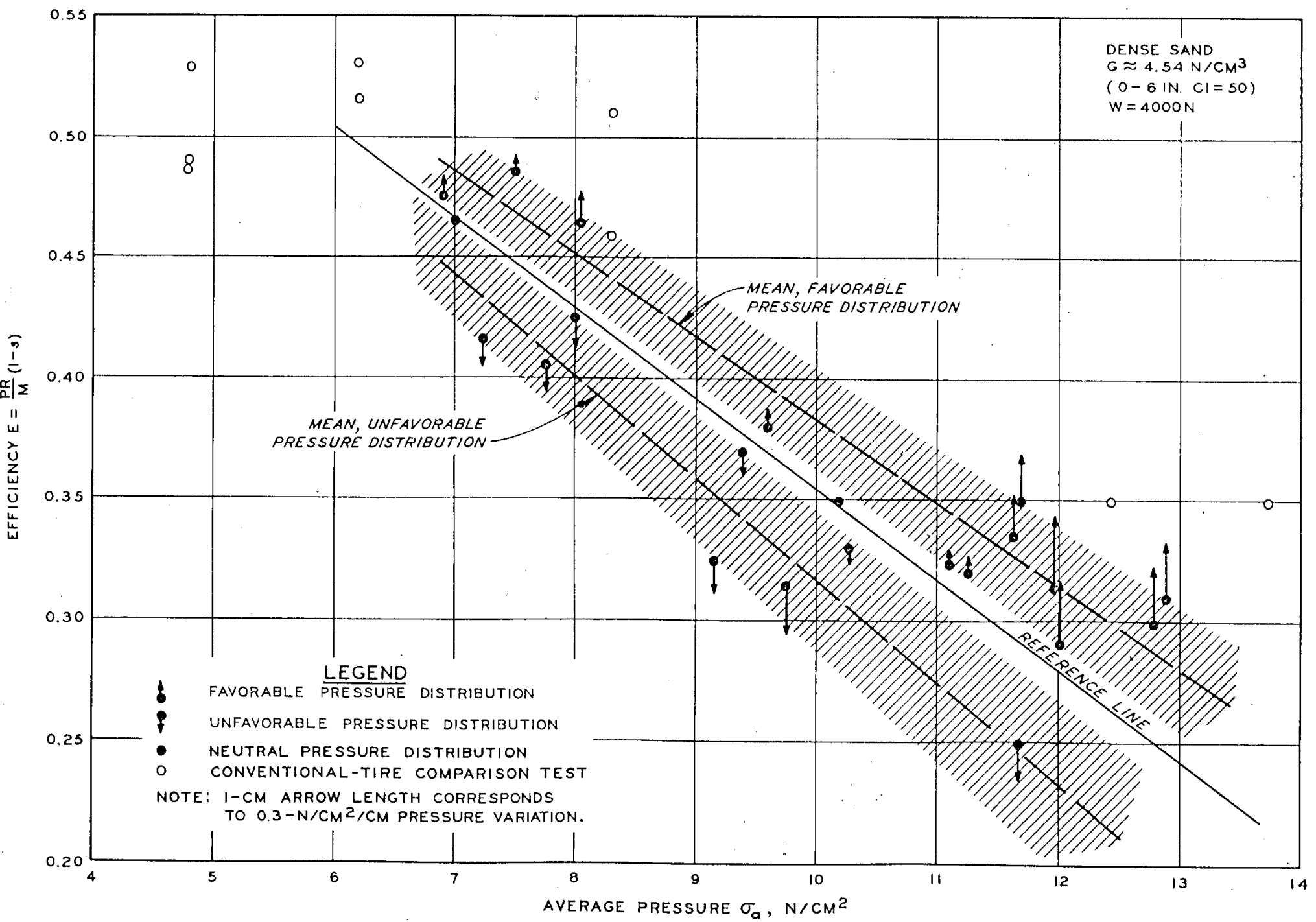

Fig. 25. Variation of efficiency; dense sand 
pressure distribution, with the neutral distribution data being almost exactly between these regions ( $f i g .25$ ).

Interpretation and discussion

82. For two reasons, the discussion of the final test results centers on the comparison of tests with favorable and unfavorable pressure distribution, i.e. without consideration of the conventional-tire tests (baseline tests):

a. The rigidity distribution for the conventional-tire tests was largely uncontrolled; therefore, there is no basis for comparison.*

b. The highly complex internal system of the experimental. Wheel constitutes a handicap that favors the conventional tires, particularly with respect to efficiency. There are no means to account qualitatively for this effect, and a correct basis for the comparison of the experimental wheel and conventional tires is thus lacking also from this point of view.

83. Furthermore, these tests have been conducted to check a principle, i.e. Whether or not wheel performance can be influenced by a control of local rigidity and to what extent. The inclusion of conventional tires (with uncontrolled rigidity distribution) in the comparison would cloud this main issue and would shift the interest to the question of whether or not conventional tires can be outperformed by the experimental wheel. Although a positive answer to this question would have given additional incentive to the test program, the performance of a conventional tire was not the issue.

84. The tires with favorable pressure distribution not only develop more pull over the total range of the conditions tested, but also do so with higher efficiency, as shown in figs. 22-25. The performance variation, expressed in percentages, is given in table 12, the reference values being the mean values between the data lines in figs. 22 and 23 and the mean values between the data scatter limits in figs. 24 and 25 , as indicated in

* See footnote on page 46. 
Table 12

Percentages of Performance Variation with Respect to the

Performance of Tires with Neutral Pressure Distribution

\begin{tabular}{|c|c|c|c|c|c|c|c|c|}
\hline \multirow{4}{*}{$\begin{array}{c}\text { Average } \\
\text { Stress, } \sigma \\
\mathrm{N} / \mathrm{cm}^{2} \\
\end{array}$} & \multicolumn{8}{|c|}{ Pressure Distribution, \% } \\
\hline & \multicolumn{4}{|c|}{ Favorable } & \multicolumn{4}{|c|}{ Unfavorable } \\
\hline & \multicolumn{2}{|c|}{$\begin{array}{l}\text { Medium-Dense } \\
\text { Sand }\end{array}$} & \multirow{2}{*}{$\frac{\text { Dense }}{\mathrm{P} / \mathrm{W}}$} & \multirow{2}{*}{ Sand } & \multicolumn{2}{|c|}{$\begin{array}{l}\text { Medium-Dense } \\
\text { Sand }\end{array}$} & \multicolumn{2}{|c|}{ Dense Sand } \\
\hline & $\mathrm{P} / \mathrm{W}$ & $\mathrm{E}$ & & & $\mathrm{P} / \mathrm{W}$ & $I$ & $\mathrm{P} / \mathrm{W}$ & $E$ \\
\hline 5 & +5.8 & +7.8 & -- & -- & -5.8 & $-3 \cdot 5$ & -- & -- \\
\hline 7 & +6.4 & +7.4 & +2.5 & $+3 \cdot 4$ & -6.4 & -3.4 & -2.5 & -5.9 \\
\hline 9 & +7.9 & +5.8 & +7.6 & +6.1 & -7.9 & -4.6 & -7.6 & -9.9 \\
\hline 11. & -- & -- & +13.0 & +9.8 & -- & -- & $-13 \cdot 0$ & $-13 \cdot 5$ \\
\hline
\end{tabular}

the figures. These reference values can reasonably be assumed to correspond to test data with neutral pressure distribution, which were, however, not numerous enough to substantiate this assumption (see fig. 25b). The performance variations generally increase (with a few exceptions) with increasing average pressure.

85. A more mearingful representation is obtained if the results with favorable pressure distribution are compared directly with those with unfavorable pressure distribution (table 13). The results in table 13 might be

Table 13

Percentages of Performance Increases with Respect to the Performance of Tires with Unfavorable Pressure Distribution

\begin{tabular}{|c|c|c|c|c|}
\hline $\begin{array}{l}\text { Average } \\
\text { Stress, } \sigma\end{array}$ & $\mathrm{P} / \mathrm{W}$ & & $\mathrm{E}_{2}$ & \\
\hline $\mathrm{N} / \mathrm{cm}^{2}$ & $\begin{array}{c}\text { Medium-Dense } \\
\text { Sand } \\
\end{array}$ & $\begin{array}{r}\text { Dense } \\
\text { Sand } \\
\end{array}$ & $\begin{array}{l}\text { Medium-Dense } \\
\text { Sand } \\
\end{array}$ & $\begin{array}{r}\text { Dense } \\
\text { Sand } \\
\end{array}$ \\
\hline 5 & +12.2 & -- & +11.9 & -- \\
\hline 7 & +13.2 & +5.6 & +11.0 & +10.0 \\
\hline 9 & +17.2 & +16.5 & $+11 \cdot 3$ & +16.5 \\
\hline 11 & -- & +29.3 & - & +27.2 \\
\hline
\end{tabular}

compared directly with the theoretical results presented in paragraphs 3238 by associating the unfavorable pressure distribution with the $\lambda=0.4$ 
condition and the favorable pressure distribution with the $\lambda=0.6$ condition. Then the order of magnitude of the performance increases derived from the theoretical study $(\mathrm{P} / \mathrm{W} \rightarrow+22.4$ percent, $\mathrm{E} \rightarrow 21.3$ percent -29 percent, paragraphs 33 and 35) becomes comparable to the experimental results in table 13. Also, the theoretically anticipated trend for less performance increase on stronger soils (paragraphs 37 and 38 ) is found to be confirmed by the experimental results (with one exception) if tests with the same average pressure are compared (table 13). These considerations are only tentatively included and do not, by any means, imply that the test conditions are quantitatively comparable to those of the theoretical study. Although the reality of the soil-wheel interaction is recognized to be far more complex than the simple assumptions of the theoretical study, the good quantitative agreement between theoretical and experimental results is thought to be noteworthy .

86. No correlation has been found between the rate of pressure variation over the contact length and the performance increase. This points to the previously discussed fact (paragraphs 18 and 19) of a certain threshold value of maximum soil reaction stresses and maximum soil stress variations. Beyond this threshold, a further increase of rigidity variation is ineffective as far as soil reactions are concerned. It is therefore concluded from the presented test data that this threshold condition was reached or passed for all tests conducted (except for the neutral pressure distribution tests, of course) so that the test results simply split into favorable and unfavorable groups without further gradual distinction. This conclusion was reached after the test program had been completed. No attempt was made to clarify this question and to identify this threshold condition quantitatively by resuming the testing, since the experimental wheel was thought to be too clumsy a machine to permit refined testing methods.

87. The variation and control of each tire pressure affect not only the rigidity distribution, but also the geometry of the soil-wheel interface. In fact, rigidity distribution and geometry cannot truly be separated. They are interdependent and can be considered the two faces of the same coin. Therefore, it might be argued that the observed performance variations are due to variation of the geometry of the soil-wheel 
interface, and not to variation of the rigidity pattern.

88. To check this argument, the test results were replotted in figs. 26 and 27 in a manner analogous to that used in figs. 22-25, except that the high and low aspect ratios, $\ell / z$, are introduced as parameters instead of favorable and unfavorable pressure distributions. The closed circles in these figures represent test results with lower-than-average and the open circles higher-than-average $\ell / z$ values. The average $l / z$
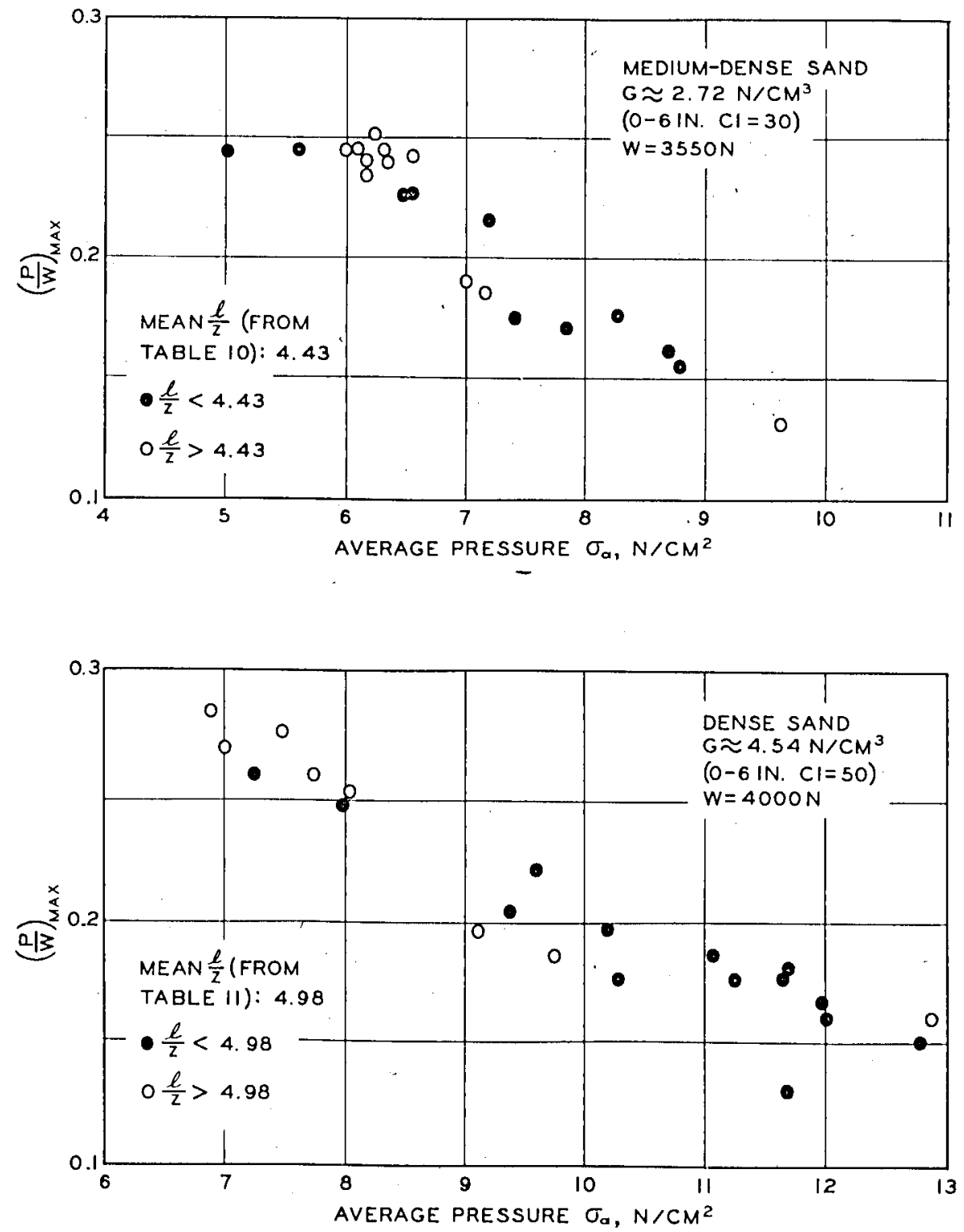

Fig. 26. Influence of aspect ratio $\ell / z$ on maximum pull/load ratio; medium-dense and dense sand 

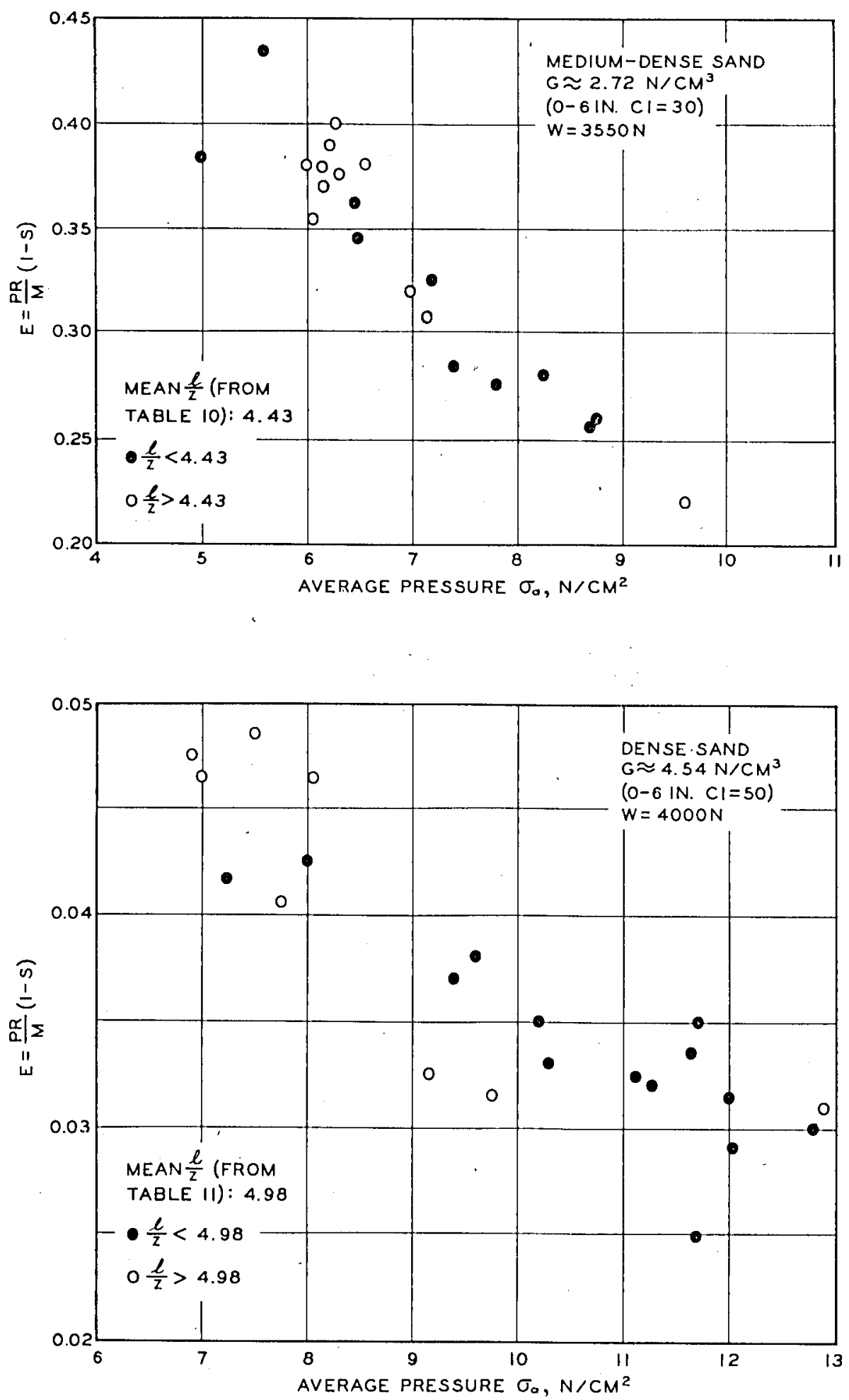

Fig. 27. Influence of aspect ratio $l / z$ on efficiency; medium-dense and dense sand 
values were determined from tables 10 and 11 as the arithmetic means, 4.43 and 4.98, for the medium-dense and dense sand tests, respectively. In figs. 26 and 27, the open and closed symbols intermix in a completely random manner, suggesting that the, $\ell / z$ value has no detectable influence on the performance of the tire. Although the $l / z$ value is recognized to be a very rough characterization of the interface geometry, and although the geometrical data collected probably are the least reliable (see paragraphs 68-70), a major trend, if present, would certainly have shown up in these plots.

89. To conclude from these results, however, that interface geometry and wheel performance are totally unrelated would be unreasonable. The interface geometry necessarily influences the wheel performance, but this effect seems to be largely offset by the predominant influence of the interface rigidity in the test conditions given. Therefore, in contrast to the usual explanation (see paragraph 5), the performance improvement of low-pressure tires is probably due mainly to an improved stress distribution, while the effect of the obviously improved interface geometry is minor. 
90. This part of the report briefly explores the possibility of applying the principle of controlled circumferential rigidity to a more effective off-road wheel. Emphasis is placed on developing the general idea presented in schematic drawings, and no attempt is made to solve the many problems of design and actual construction of such a wheel. The point is stressed that the pure realization of the principle of controlled rigidity, i.e. a wheel working without additional energy input, does not seem possible for practical purposes. Any rationally designed wheel with controlled local rigidity will have to rely on an auxiliary power source in addition to the axle torque input. It then becomes rather difficult, if not impossible, to split the performance increase accurately into the fraction caused by the rigidity control and that caused by increased energy input. For this reason, the experimental wheel reported herein had to be a pure, but highly impractical, realization of the original idea of controlled rigidity. The experimental validation of this principle would not have been possible with the wheel proposed in this part of the report.

91. Throughout this report the terms "Iocal rigidity" and "local internal pressure" have been used synonymously in connection with the experimental wheel.* The straightforward application of this idea leads to a. multicompartment tire with inflation pressure control for each individual compartment.

92. A simple device of this kind is shown schematically in fig. 28 . The rotating wheel slides against a nonrotating circular pressure distributor provided with airflow channels that are connected to a compressor.

* This implies a dimensionally correct definition of rigidity. Rigiditý generally is defined as the resistance of a system to deformation. If a single force is applied, the dimension of rigidity is force/length $(\mathrm{N} / \mathrm{cm})$; if a stress is applied, rigidity has the dimension of stress/ length $(\mathrm{N} / \mathrm{cm} 3)$. The definition and dimension of rigidity are system dependent. Rigidity might as well be defined as the resistance to deformation per some characteristic length. In this case, the dimension of rigidity is $\mathrm{N} / \mathrm{cm}^{2}$, which justifies the synonymous use of pressure and rigidity. As characteristic length, tire section height or wheel radius might be chosen. 

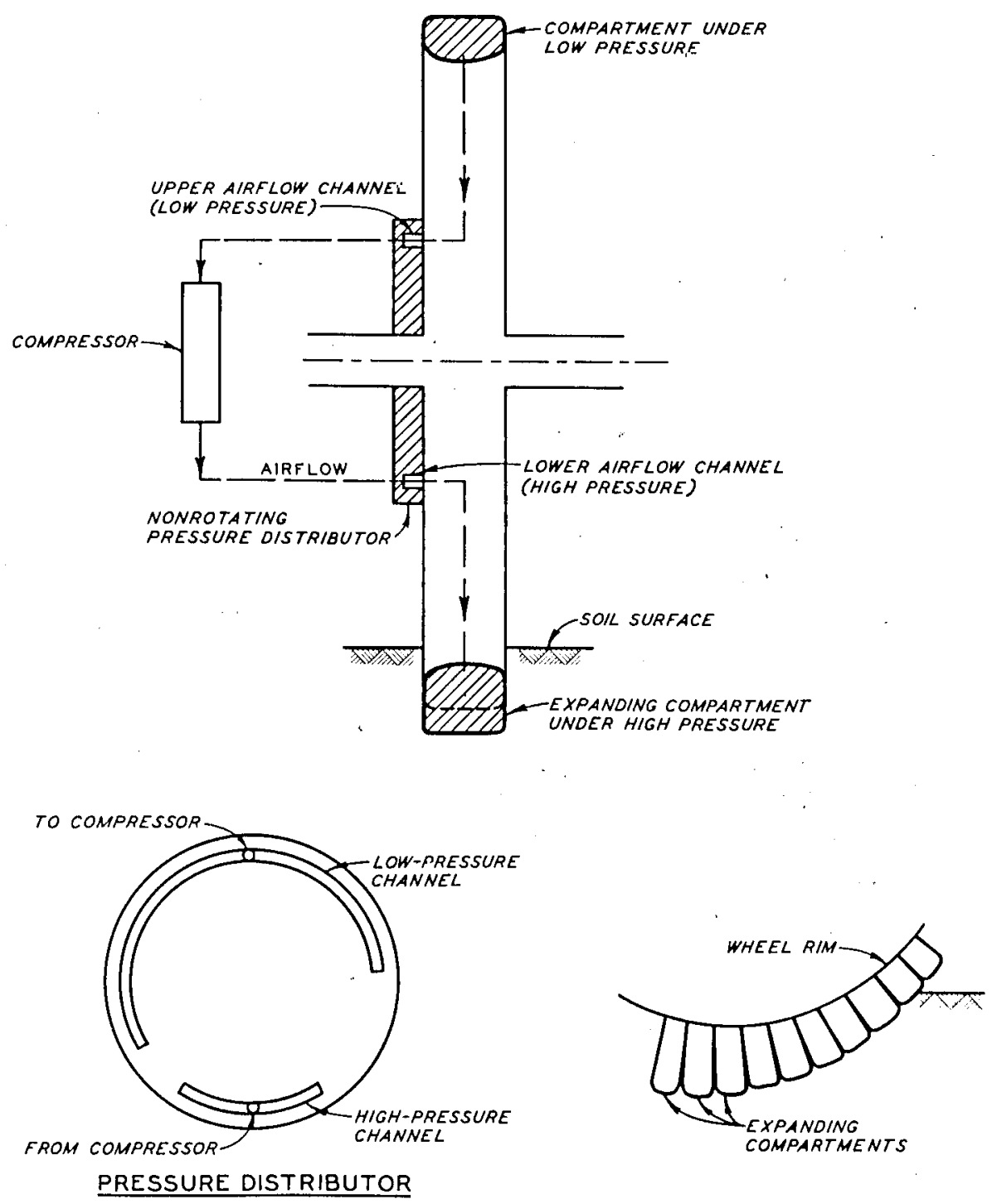

Fig. 28. Schema of a multicompartment tire

The compartments of the tire are connected to these airflow channels by individual inlets moving along the channels as the wheel rotates. While a compartment is in contact with the lower, high-pressure channel that corresponds to the interface position, the pressure of that compartment increases as it passes along the soil-wheel interface. After the considered compartment leaves the interface, its inlet comes into contact with the 
upper, low-pressure channel so that the original low pressure of the compartment is restored.

93. The proposed design does not allow an individual pressure control in each compartment--this would require a much more sophisticated device--but it assures a roughly linear increase of the internal tire pressure (rigidity) along the interface. The rate of pressure increase over the contact length is a function of rotational velocity, air pressure, and cross section of the air ducts within the wheel. Therefore, the overall pressure distribution within the contact zone can be controlled by varying one or more of these parameters, preferably the air pressure of the airflow channels. A more sophisticated air-pressure distribution control could be obtained by regulating the individual air inlets. This would permit experimental identification of the optimum rigidity pattern within the frame of a general pressure increase.

94. The tests with the experimental wheel showed that the threshold for the rigidity increase over the interface on sand is not very high. Therefore, the energy consumption of the compressor and the maximum pressure in each compartment probably will not be excessive. For this reason, it appears feasible to protect the compartments from popping out by wrapping the "tire" in a ductile membrane, or by some other simple means. Furthermore, the airflow through the wheel-compressor system operates as a closed circuit, which also reduces the energy consumption of the compressor.

95. The concept of a multicompartment tire, as outlined above, was developed from the consideration of how wheel performance on soft, level soil surfaces could be improved. The proposed design is a fairly good material realization of the segmented wheel concept, recently developed as a mathematical model to study dynamic ride characteristics of vehicles on rough terrain. 9 In this model, the tire deformation properties are represented by a radial arrangement of springs (segments) of equal stiffness equivalent to the uniform inflation pressure. The segments correspond to the compartments of the multicompartment tire, and the spring stiffness to the air pressure for each compartment.

96. Undoubtedly, a variation of the spring stiffness within the contact zone will, more or less, affect the dynamic ride characteristics of 
the wheel. If, in a computer simulation with the segmented wheel model, a pressure distribution could be determined that significantly improves ride dynamics, it would be an additional incentive to actually build and use the proposed wheel. There are reasons to believe that this pressure variation is similar to the favorable pressure distribution for soft-soil performance improvements, i.e. increasing from the forward to the rearward edge of the interface. The relatively soft forward edge of the interface tends to attenuate the first shock produced by an encountered ground irregularity; the shock is then increasingly dampened as the stiffer compartments come into contact with the obstacle.

97. The proposed multicompartment tire will not suffer from the handicap (all too familiar in mobility research) of being an overspecialized device that fails on highways. If equal pressure is applied in all compartments, the tire performance probably will not be too different from that of normal tires.

98. Finally, reference is made to two papers in. which the idea of multicompartment tires has been advocated, in a quite different context, however. Mcleod ${ }^{10}$ proposes to influence the pressure distribution across the wheel by means of an "annular compartment tire" to improve the loadcarrying capacity of the wheel on layered soils. In reference 11 , development of a multicompartment tire similar to the one proposed herein and tests with a model tire are described. However, the purpose of this latter construction was to replace the conventional engine and gear box of vehicles and to make the wheel itself the propelling unit by providing the driving power directly at the wheel periphery. In fact, the compartments. of this tire are combustion chambers. This solution requires extremely high pressure and temperature resistance of the tire material and, therefore, is not practical at the present time.

99. Although based on different starting considerations, the multicompartment tires, as proposed in reference 11 and in this report, work with increasing tire pressure from the forward to the rearward edge of the interface. Thus, there are no conflicting requirements, $i . e$. the additional energy necessary to obtain the favorable pressure distribution also acts in a favorable manner from the point of view developed in reference 11 
in that it directly propels the wheel. So the advantages of the wheel. design developed herein are twofold: favorable pressure distribution and favorable energy distribution at the soil-wheel interface. They may be threefold if the proposed dynamic studies show this favorable pressure distribution to be beneficial also from a ride dynamics viewpoint. 


\section{PART VI: CONCLUSIONS AND RECOMMENDATIONS}

\section{Conclusions}

100. The test device does not permit quantitative assessments, but the following conclusions are believed to be warranted by the findings of this study:

a. Maximum pull and efficiency of tires on sand depend on the local internal tire pressure distribution within the soilwheel interface.

b. The control of this pressure distribution is an effective means of improving the performance of wheels on sand.

c. The pressure distribution that continuously increases from the forward to rearward edge of the interface is favorable. The wheel then not only develops more pull, but also operates at higher efficiency.

d. For maximum performance increase, only a slight rate of pressure variation is necessary for the tested conditions.

e. Effects of interface geometry on pull and efficiency are negligible for the tested conditions.

f. There are no theoretical reasons that exclude the principle of controlled tire pressure (rigidity) distribution from application to off-road mobility in sand.

\section{Recommendations}

101. It is recommended that:

a. Similar tests with the experimental wheel be conducted in cohesive soils to broaden the spectrum of possible applications.

b. Computer studies be made with the segmented-wheel model to assess the possibly favorable effects on ride dynamics of increasing tire pressure distribution.

c. A prototype of the proposed multicompartment tire be constructed to obtain quantitative assessments of tire performance variations and limitations. 
1. Wiendieck, K. W., "Contribution to the Mechanics of Rigid Wheels on Sand," Technical Report M-68-2, May 1968, U. S. Army Engineer Waterways Experiment Station, CE, Vicksburg, Miss.

2. Green, A. J., Jr. and Murphy, N. R., Jr., "Stresses Under Moving Vehicles; Distribution of Stresses Beneath a Towed Pneumatic Tire in Air-Dry Sand," Technical Report No. 3-545, Report 5, July 1965, U. S. Army Engineer Waterways Experiment Station, CE, Vicksburg, Miss.

3. Freitag, D. R., Green, A. J., and Murphy, N. R., "Normal Stresses at the Tire-Soil Interface in Yielding Soils," Soil Stresses, Foundation Settlement, and Caisson Stability, Record No. 74, pp 1-18, 1965, Highway Research Board, Washington, D. C.

4. Freitag, D. R. and Green, A. J., "Distribution of Stresses on an Unyielding Surface Beneath a Pneumatic Tire," Stress Distribution in Earth Masses, Bulletin 342, pp 14-23, 1962, Highway Research Board, Washington, D. C.

5. VandenBerg, G. E. and Gill, W. R., "Pressure Distribution Between a Smooth Tire and the Soil," Transactions of the ASAE, American Society of Agricultural Engineers, Vol 5, No. 2, 1962, pp 105-107.

6. Leflaive, E. M., "Mechanics of Wheels on Soft Soils; A Method of Analyzing Test Results," Technical Report No. 3-729, Report 1, June 1966, U. S. Army Engineer Waterways Experiment Station, CE, Vicksburg, Miss.

7. Wong, J. Y. and Reece, A. R., "Prediction of Rigid Wheel Performance Based on the Analysis of Soil-Wheel Stresses; Part I, Performance of Driven Rigid Wheels," Journal of Terramechanics, Vol 4, No. 1, 1967 , pp $81-98$.

8. McRae, J. L., Powell, C. J., and Wismer, R. D., "Performance of Soils Under Tire Loads; Test Facilities and Techniques," Technical Report No. 3-666, Report 1, Jan 1965, U. S. Army Engineer Waterways Experiment Station, CE, Vicksburg, Miss.

9. Lessem, A. S., "Dynamics of Wheeled Vehicles; A Mathematical Model for. the Traversal of Rigid Obstacles by a Pneumatic Tire," Technical Report M-68-1, May 1968, U. S. Army Engineer Waterways Experiment Station, CE, Vicksburg, Miss.

10. Mcleod, N. W., "Influence of Multiple Compartment Tires on Off-theRoad Mobility," Proceedings of the lst International Conference on the Mechanics of Soil-Vehicle.Systems, Paper No. 37, June 1961, pp 575-586.

11. "A New Type of Vehicle Drive," Journal of Terramechanics, Vol I, No. 3, 1964, pp 83-90. 



\section{APPENDIX A: SIMPLIFIED GRAPHIC SOLUTION FOR}

DETERMINATION OF INTERNAL FORCES*

1. Decompose the forces $P$ into normal (N) and tangential (T) components (fig. 14). Choose a convenient scale to represent the forces by a length.

2. Compute the force $S_{1}$. from the strain gage readings:

$$
S_{I}=\frac{S}{2 \sin \theta}
$$

3. Starting from a point, $\mathbb{M}$ (fig. 16), draw all $\mathrm{S}$-lines parallel to the corresponding lines in the static system.

4. Transpose the length $S_{1}$; reduce this length by the length $T_{I}$; and mark the point $\mathrm{Q}_{1}$.

5. Draw the line $N_{1}$ from the point $Q_{I}$, parallel to $N_{I}$ in the static system, and transpose the corresponding length from the point $\mathrm{Q}_{1}$.

6. The $\mathbb{N}_{1}$ line cuts the $S_{2}$-line in a point $U_{1}$ : Denote the remaining free length of $N_{1}$ as $R_{1}$.

7. Reduce the $\mathrm{S}_{2}$-line, counting from $\mathrm{U}_{1}$ toward $\mathrm{M}$, by $\mathrm{k}_{\mathrm{S}} \mathrm{R}_{1}+\mathrm{T}_{2}$, $\mathrm{k}_{\mathrm{s}}$ being a factor. Choose $\mathrm{k}_{\mathrm{s}}=0.1$ for the first trial. Mark the thus obtained point on the $S_{2}$-line $Q_{2}$. (Attention: $T_{1}$ always has to be transposed according to its sign. $\mathrm{T}_{6}$ is almost always negative and the $S_{6}$-line thus has to be prolonged beyond the point $U_{6}$ instead of being reduced. )

8. Repeat steps 5-7, starting with $Q_{2}$, and thus determine succes- . sively $R_{2}, R_{3}, R_{4}, R_{5}$, and $R_{6} \cdot R_{6}$ is always equal to $N_{6}$.

9. Compute $\mathrm{k}_{\mathrm{s}} \mathrm{R}_{6}\left(=0.1 \mathrm{R}_{6}\right)$ and compare it with the length $\bar{M}_{6}$. If $0.1 R_{6}<\overline{M Q}_{6} \pm 20$ percent, the whole operation is to be repeated with a slightly increased $k_{s}$ value $\left(k_{s}=0.12\right)$. If $0.1 R_{6}>\overline{M Q}_{6} \pm 20$ percent, repeat with a decreased $k_{s}$ value $\left(k_{s}=0.08\right)$. If $k_{s} R_{6}=\overline{M Q}_{6} \pm 20$ percent, the approximation is considered sufficient.

* Reference figs. 14 and 16 in main text of this report. 

Commanding General, U. S. Army Materiel Command

ATIN: AMCRD-DM

AMCRD-TV

AMCRD-G

Washington, D. C.

1

2

1

1

Commanding office

Hanover, N. H.

Commanding Officer, U. S. Army Mobility Equipment

Research and Development Center

ATIN: Technical Documents Center, Building 315

Fort Belvoir, Va.

Commanding Officer, USA Electronics Command

1

Meteorological Support Activity

Fort Huachuca, Ariz.

Commanding Officer, USA Electronics Research and

Development Laboratories

ATTN: SELRA/ADT

Fort Monmouth, N. J.

Chief of Research and Development

1

ATIN: Chief, Combat Materiel Division

Department of the Army

Washington, D. C.

Chief of Research and Development

ATIT: CRDES, Department of the Army

Washington, D. C.

Chief of Research and Development

Headquarters, Department of the Army

ATIN: Director of Army Technical Information

Washington, D. C.

3 copies

of Form

1473

Defense Intelligence Agency

ATTN: DIAAP-4B

Washington, D. C.

Special Assistant for Environmental Services

Joint Chiefs of Staff

Washington, D. C. 
Director, OSD/ARPA Regional Field Office ARMISH-MAAG (RFO-I)

APO New York 09205

Commanding Officer, U. S. Army Arctic Test Center

APO Seattle, Wash.

Chief of Engineers

ATIN: ENGME-RD

ENGAS-I

ENGMC-ER

ENGME-RO (for Engineer Standardization Program)

ENGSA (Dr. G. G. Quarles)

Department of the Army

Washington, D. C.

War Plans Division, Engineer Strategic Studies Group

Office, Chief of Engineers, U. S. Army

Erskin Ha.l1, Army Map Service

Washington, D. C.

Commander, U. S. Army Combat Development Command Engineer Agency ATIN: CSGEN-M, Fort Belvoir, Va.

The Librarian, U. S. Army Engineer School Library, Thayer Hall Fort Belvoir, Va.

COL John R. Oswalt, Jr., Director

U. S. Army Engineer Topographic Laboratories, Fort Belvoir, Va.

USA Engineer School, ATIN: Heavy Construction Section, Dept of

Engrg Pavements and Materials Group

Fort Belvoir, Va.

Commanding General, XVIII Airborne Corps

ATrN: Corps Engineer

Fort Bragg, N. C.

Commanding Officer, 326th Engineer Battalion

Fort Campbell, Ky.

Senior Engineer Instructor, Office of Military Instruction

U. S. Continental Army Command Intelligence Center

Fort Bragg, N. C. 
President, U. S. Army Armor Board

Fort Knox, Ky.

President, U. S. Army Artillery Board

Fort Sill, Okla.

President, U. S. Army Infantry Board

Fort Benning, Ga.

Commanding General, U. S. Army Weapons Command

ATIN: AMSWE-RDR

Rock Island, Ill.

Commanding General, U. S. Army Tank-Automotive Command ATTN: AMSTA-BSL

Warren, Mich.

Commander, U. S. Army Forces Southern Command

ATTN: Engineer

Fort Amador, Canal Zone

Commanding General, U. S. Continental Army Command

Engineer Division, DCSLOG

ATTIN: ATLOG-E-MB

Fort Monroe, Va.

Commanding General, U. S. Continental Army Command

ATTN: ATIT-RD

Fort Monroe, Va.

Commandant, Command and General Staff College

ATTN: Archives

Fort Leavenworth, Kans.

Commanding General, U. S. Army Tank-Automotive Command Land Locomotion Division

ATTN: AMSTA-UL

Warren, Mich.

Commander, U. S. Army Picatinny Arsenal

Dover, N. J.

Commanding Officer, Yuma Proving Ground ATTN: STEYP-TGM

Yuma, Ariz. 
Technical Library, Branch No. 4

U. S. Army Limited War Laboratory

Aberdeen Proving Ground, Md.

Automotive Engineering Laboratory

ATTN: STEAP-DP-LU

Aberdeen Proving Ground, Md.

Director, Development and Proof Services

ATTN: STEAP-DS-TU

Aberdeen Proving Ground, Ma.

Commanding officer

ATTN: Tech Library, Bldg 313

Aberdeen Proving Ground, Ma.

Commanding Officer, U. S. Army General Equipment Test Activity Fort Lee, Va.

Chief, Crops Division, U. S. Army Biological Laboratories

Fort Detrick, Md.

Commanding Officer, U. S. Army Combat Developments

Command Transportation Agency

ATTN: Mr. EarI S. Brown

Fort Eustis, Va.

Asst. Chief of Staff for Force Development

Hqs, Department of the Army, ATTN: FOR DS SSS

Washington, D. C.

United States Army Attaché, American Fmbassy

U. S. Navy 100, Box 36

Fleet Post Office

New York, N. Y.

Office of Naval Research

ATIN: Geography Branch

Department of the Navy

Washington, D. C.

Commanding Officer, PHIBCB Two

U. S. Naval Amphibious Base

Little Creek, Norfolk, Va.

U. S. Naval Academy

ATTN: Library, Serials Division

Annapolis, Md. 21402 
Commanding Officer, PHIBCB One

U. S. Naval Amphibious Base

Coronado, San Diego, Calif.

Commanding Officer and Director

Naval Civil Engineering Laboratory

Port Hueneme, Calif.

Director, Naval Warfare Research Center

Stanford Research Institute

Menlo Park, Calif.

Commander, Naval Facilities Engineering Command

Navy Department, Code 03

Washington, D. C.

Commanding Officer, U. S. Naval Photographic Interpretation Center

Washington, D. C.

Chief, Combat Service Support Division

Marine Corps Landing Force Development Center

Marine Corps Schools

Quantico, Va.

Commander, 3800th $\mathrm{AB}$ Wing, $\mathrm{AU}$

ATTN : BDCE-ED

Maxwell AFB, Ala.

Headquarters, USAF

(AFRSTC) Astronautics Division

DCS/Research and Development

Washington, D. C.

Commander, U. S. Strike Command

ATIN: J4-E

McDill AF'B, Fla.

Chief, Concepts and Evaluation Group

U. S. Strike Command

McDill AFB, Fla.

Commander, ADTC

ATTN: ADBPS-12

Eglin AFB, Fla.

Dr. Nicholas C. Costes, Space Sciences Laboratory, Code RSSL-N Marshall Spaceflight Center

Huntsville, Ala. 
Headquarters, Air Force Systems Command

Directorate of Civil Engineering (SCOC)

Andrews AFB, Washington, D. C. .

Headquarters, Tactical Air Command

ATTN: DEPE

Langley AFB, Va.

Headquarters, U. S. Air Force

Director of Civil Engineering (AFOCE-KA)

Washington, D. C.

Headquarters, USAF

Base Structures Branch, Directorate of Civil Engineering

ATTN: AFOCE-GC

Washington, D. C.

Commander, Hqs, Military Airlift Command

ATIN: MAMCE 7 FS

Scott AFB, III.

Commander, ASD (ASNMS-20)

Wright-Patterson AFB, Ohio

Air Force Weapons Laboratory

ATTN: Civil Engineering Branch, WLDC

Kirtland $\mathrm{AFB}$, N. Mex.

Terrestrial Sciences Lab (CRJT)

Air Force Cambridge Res Lab, I. G. Hanscom Field

Bedford, Mass.

Library, Division of Public Documents

U. S. Government Printing Office

Washington, D. C.

Library of Congress, Documents Expediting Project

Washington, D. C.

Defense Documentation Center

ATTIN: Mr. Myer Kahn

Cameron Station

Alexandria, Va.

National Tillage Machinery Laboratory

U. S. Department of Agriculture

Auburn, Ala. 
Chief, World Soil Geography Unit, Soil Conservation Service USDA, Room 233A Federal Center Bldg

Hyattsville, Md.

Chief, Crops Protection Branch

Crops Research Division, Agricultural Research Service

Beltsville, Ma.

Director, Pacific Southwest Forest and Range Experiment Station ATTM: Henry W. Anderson

Berkeley, Calif.

U. S. Geological Survey

Chief, Source Material Unit, Branch of Military Geology

Washington, D. C.

Coastal Studies Institute

Louisiana State University

Baton Rouge, La.

Iibrarian, Bureau of Public Roads

1

U. S. Department of Commerce

Washington, D. C.

New York University

ATIN: Engineering Library.

University Heights, Bronx, N. $Y$.

Professor L. C. Stuart

University of Michigan

Ann Arbor, Mich.

University of Arkansas, College of Engineering

ATTN: Mr. Henry H. Hicks, Jr.

Fayetteville, Ark.

Stanford Research Institúte

ATTN: Mr. Gordon S. Wiley

Menlo Park, Calif. 
Engineering Societies Library

New York, N. Y.

Highway Research Board

Washington, D. C.

Research Analysis Corporation

ATTN: Library

McLean, Va. 22101

Davidson Laboratory, Stevens Institute of Technology

ATTI: Dr. I. R. Ehrlich

Hoboken, N. J.

Center for Research and Engineering Science

University of Kansas

Lawrence, Kansas

Mr. David Sloss, Civil Engineering Department

University of Detroit

Detroit, Mich. 48221

Institute of Transportation and Traffic Engineering Library

1

University of California

Richmond, Calif. 94804

Wilson, Nuttall, Raimond Engineers, Inc.

ATTN : Library

Chestertown, Ma.

Cornell Aeronautical Laboratory, Inc.

P. O. Box 235

Buffalo, N. Y. 14221 
Unclassified

Security Classification

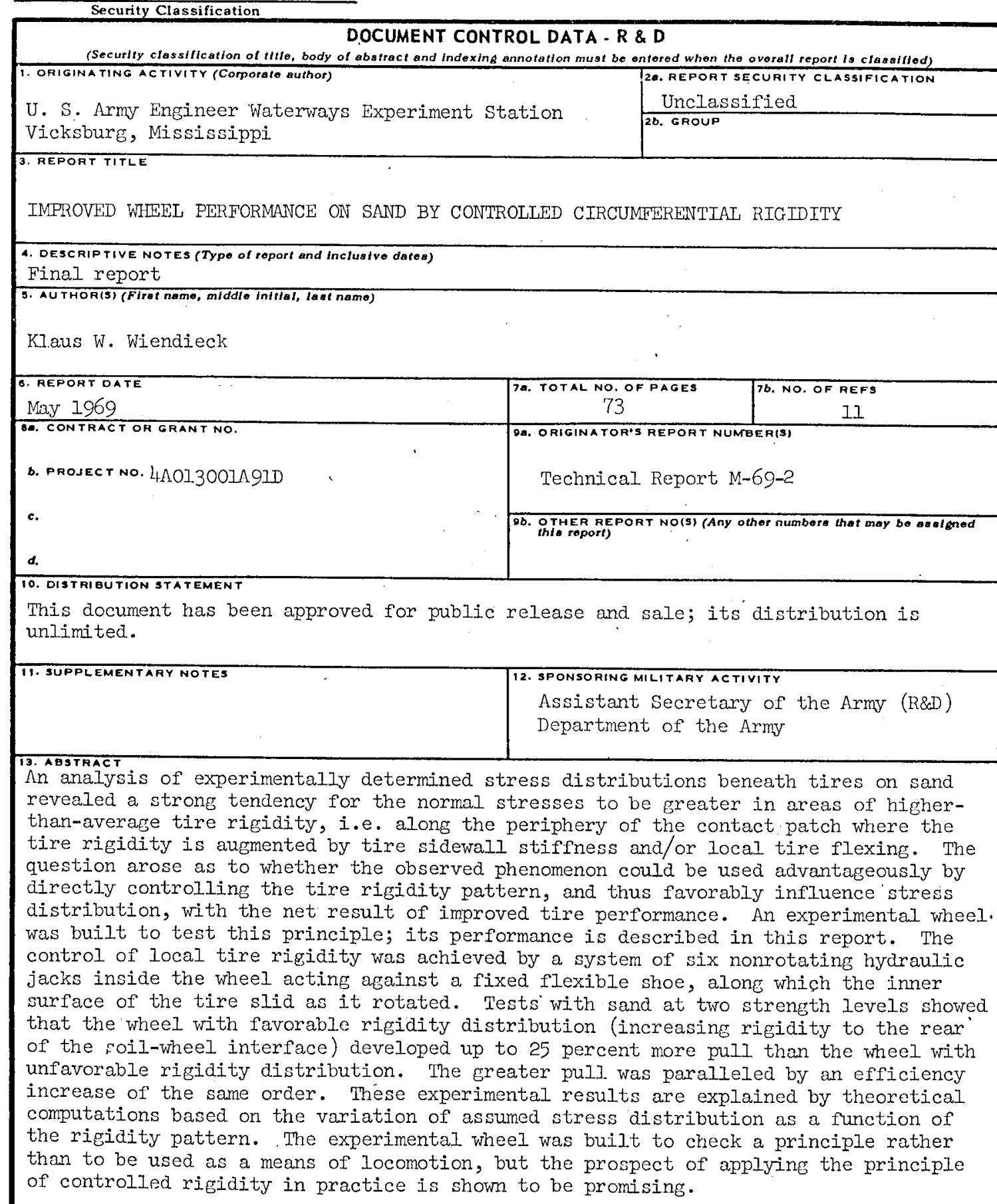


Unclassified

Security Classification

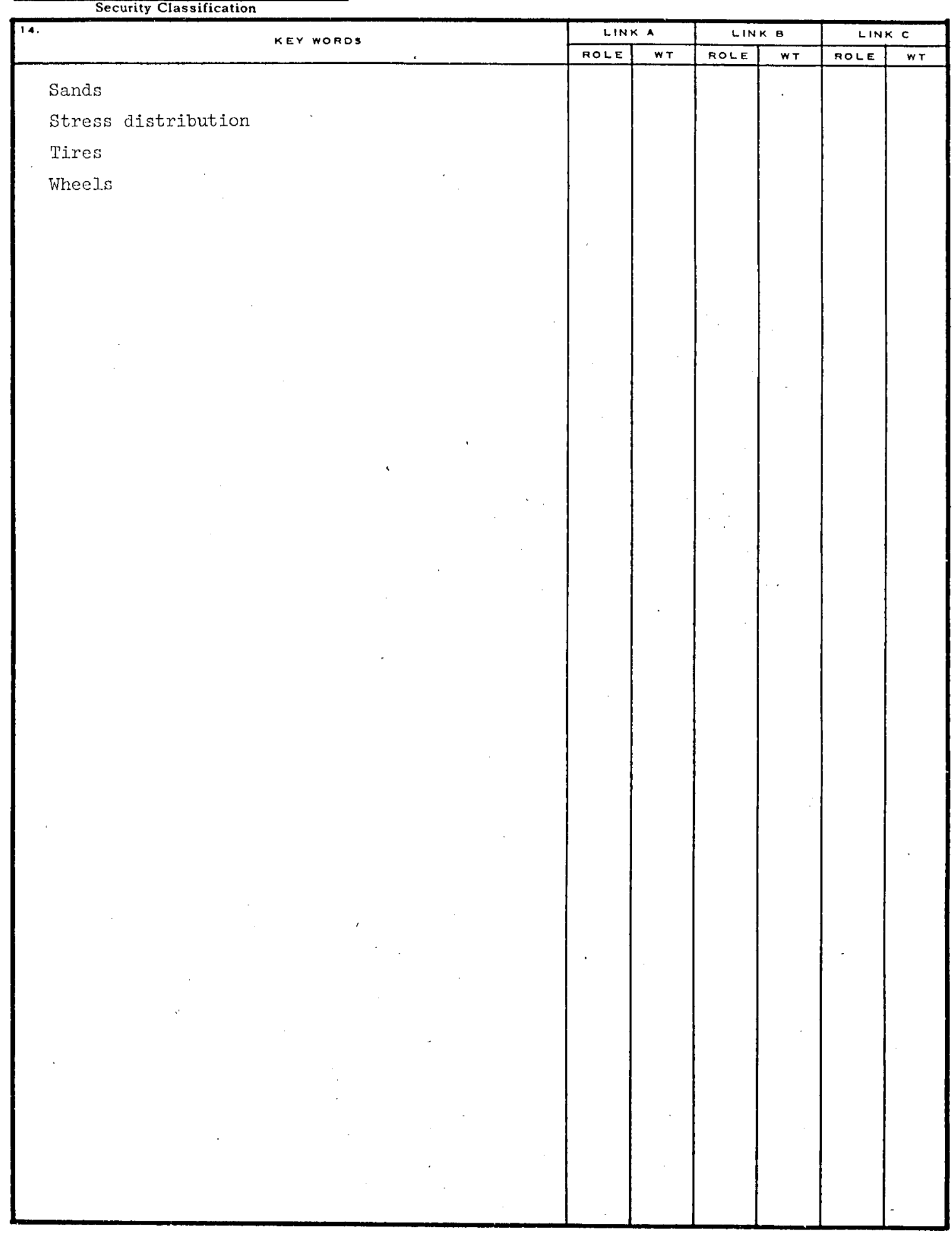

Unclassified

Security Clessification 
, 